\title{
ZERO MACH NUMBER LIMIT IN CRITICAL SPACES FOR COMPRESSIBLE NAVIER-STOKES EQUATIONS
}

\author{
BY RAPHAËL DANCHIN
}

ABSTRACT. - We are concerned with the existence and uniqueness of local or global solutions for slightly compressible viscous fluids in the whole space. In [6] and [7], we proved local and global well-posedness results for initial data in critical spaces very close to the one used by $\mathrm{H}$. Fujita and T. Kato for incompressible flows (see [14]). In the present paper, we address the question of convergence to the incompressible model (for ill-prepared initial data) when the Mach number goes to zero. When the initial data are small in a critical space, we get global existence and convergence. For large initial data and a bit of additional regularity, the slightly compressible solution is shown to exist as long as the corresponding incompressible solution does. As a corollary, we get global existence (and uniqueness) for slightly compressible two-dimensional fluids.

(C) 2002 Éditions scientifiques et médicales Elsevier SAS

RÉSUMÉ. - On étudie l'existence et l'unicité de solutions locales ou globales pour les fluides légèrement compressibles dans l'espace entier. Dans [6] et [7], on a montré que le modèle était bien posé dans des espaces critiques très proches de ceux utilisés par H. Fujita and T. Kato pour les fluides incompressibles (voir [14]). Dans cet article, on étudie la convergence vers le modèle incompressible (pour des données initiales mal préparées) lorsque le nombre de Mach tend vers zéro. On obtient un résultat d'existence et de convergence global en temps pour des données initiales petites et à régularité critique. Pour des données grandes mais un peu plus régulières, la solution du système légèrement compressible existe aussi longtemps que la solution incompressible correspondante. En particulier, on a existence (et unicité) globale en dimension deux pour les fluides légèrement compressibles.

(C) 2002 Éditions scientifiques et médicales Elsevier SAS

\section{Introduction}

The motion of a slightly compressible barotropic fluid is described by the following system:

$\left(N S C^{\varepsilon}\right)\left\{\begin{array}{l}\partial_{t} \rho^{\varepsilon}+\operatorname{div} \rho^{\varepsilon} u^{\varepsilon}=0, \\ \partial_{t}\left(\rho^{\varepsilon} u^{\varepsilon}\right)+\operatorname{div}\left(\rho^{\varepsilon} u^{\varepsilon} \otimes u^{\varepsilon}\right)-\mu \Delta u^{\varepsilon}-(\lambda+\mu) \nabla \operatorname{div} u^{\varepsilon}+\frac{\nabla P}{\varepsilon^{2}}=\rho^{\varepsilon} f^{\varepsilon}, \\ \left.\left(\rho^{\varepsilon}, u^{\varepsilon}\right)\right|_{t=0}=\left(\rho_{0}^{\varepsilon}, u_{0}^{\varepsilon}\right) .\end{array}\right.$

Here $\rho^{\varepsilon}=\rho^{\varepsilon}(t, x) \in \mathbb{R}^{+}$and $u^{\varepsilon}=u^{\varepsilon}(t, x) \in \mathbb{R}^{N}$ stand for the dimensionless density and velocity field, and the pressure $P$ is a suitably smooth function of $\rho^{\varepsilon}$. Unless otherwise specified, it will always be assumed that $x$ belongs to the whole space $\mathbb{R}^{N}(N \geqslant 2)$. The case of periodic boundary conditions $x \in \mathbb{T}^{N}$ will be investigated in a forthcoming paper. We denote by $\lambda$ and $\mu$ the two Lamé coefficients of the fluid, which are constant and satisfy $\mu>0$ and $\nu \stackrel{\text { def }}{=} \lambda+2 \mu>0$. Such a condition ensures ellipticity for the operator $\mu \Delta+(\lambda+\mu) \nabla$ div and is satisfied in the 
physical cases (where $\lambda+2 \mu / N \approx 0$ ). The initial conditions $\left(\rho_{0}^{\varepsilon}, u_{0}^{\varepsilon}\right)$ and the external force $f^{\varepsilon}$ are given.

The system above is obtained by rewriting the compressible Navier-Stokes equations in dimensionless form. The parameter $\varepsilon$, called Mach number, is given by $\varepsilon=L T^{-1} \chi^{-1}$ where $L$ and $T$ are the typical values of length and time (before rescaling) and $\chi$ stands for the sound speed. The rescaled density $\rho^{\varepsilon}$ is given by $\rho / \bar{\rho}$ where $\rho$ is the density of the fluid and $\bar{\rho}$, its typical value. Therefore, the typical value of $\rho^{\varepsilon}$ is one. One shall further assume that $\rho^{\varepsilon}$ tends to 1 at infinity and that the (rescaled) pressure satisfies $P^{\prime}(1)=1$. More explanations on the derivation of the above model may be found in [17,18] or in the introduction of [25].

A large amount of literature has been devoted to the existence of solutions for $\left(N S C^{\varepsilon}\right)$ and to the convergence of $\left(\rho^{\varepsilon}, u^{\varepsilon}\right)$ when $\varepsilon$ goes to zero. Roughly, two different heuristics have been used. The case of well-prepared data which corresponds to the assumption that $\rho_{0}^{\varepsilon}=1+\mathrm{O}\left(\varepsilon^{2}\right)$ and $\operatorname{div} u_{0}=\mathrm{O}(\varepsilon)$ has been investigated in [21,22,24] and [18].

In the present work, we shall concentrate on the case of ill-prepared data, where it is only assumed that $\rho_{0}^{\varepsilon}=1+\varepsilon b_{0}^{\varepsilon}$ with $\left(b_{0}^{\varepsilon}, u_{0}^{\varepsilon}, f^{\varepsilon}\right)$ uniformly bounded (in a convenient functional space), $\mathcal{P} u_{0}^{\varepsilon}$ tending to some $v_{0}$ and $\mathcal{P} f^{\varepsilon}$ tending to some $g$ when $\varepsilon$ goes to $0 .{ }^{1}$ If we set $\rho^{\varepsilon}=1+\varepsilon b^{\varepsilon}$, we are led to study

$$
\left\{\begin{array}{l}
\partial_{t} b^{\varepsilon}+\frac{\operatorname{div} u^{\varepsilon}}{\varepsilon}=-\operatorname{div}\left(b^{\varepsilon} u^{\varepsilon}\right), \\
\partial_{t} u^{\varepsilon}+u^{\varepsilon} \cdot \nabla u^{\varepsilon}-\frac{\mu \Delta u^{\varepsilon}+(\lambda+\mu) \nabla \operatorname{div} u^{\varepsilon}}{1+\varepsilon b^{\varepsilon}}+\frac{P^{\prime}\left(1+\varepsilon b^{\varepsilon}\right)}{1+\varepsilon b^{\varepsilon}} \frac{\nabla b^{\varepsilon}}{\varepsilon}=f^{\varepsilon} \\
\left.\left(b^{\varepsilon}, u^{\varepsilon}\right)\right|_{t=0}=\left(b_{0}^{\varepsilon}, u_{0}^{\varepsilon}\right) .
\end{array}\right.
$$

One expects $u^{\varepsilon}$ to tend to $v$ where $v$ solves the incompressible Navier-Stokes equations:

$$
\left\{\begin{array}{l}
\partial_{t} v+v \cdot \nabla v-\mu \Delta v+\nabla \Pi=g, \\
\operatorname{div} v=0 \\
\left.v\right|_{t=0}=v_{0}
\end{array}\right.
$$

which may be rewritten

$$
\left\{\begin{array}{l}
\partial_{t} v+\mathcal{P}(v \cdot \nabla v)-\mu \Delta v=g \\
\left.v\right|_{t=0}=v_{0}
\end{array}\right.
$$

The expected convergence however is not easy to justify rigorously. The main difficulty is that one has to face the propagation of acoustic waves with the speed $\varepsilon^{-1}$, a phenomenon which does not occur in the case of "well-prepared" data.

Nevertheless, several remarkable results have been obtained recently. First of all, for initial data with minimal regularity assumptions, P.-L. Lions stated in [26] the existence of global weak solutions in the energy space for compressible Navier-Stokes equations. The pressure law considered is of type $P(\rho)=a \rho^{\gamma}$ with certain restrictions on $\gamma$ depending on the space dimension $N$. Since then, convergence results to the incompressible model have been proved by B. Desjardins, E. Grenier, P.-L. Lions and N. Masmoudi. The case of periodic boundary conditions has been investigated in [27], the case of bounded domains with Dirichlet conditions in [12] and the case of the whole space in [11]. Some local weak convergence results are also available in a more general context (see [28]). Roughly, the main difference between the whole

\footnotetext{
${ }^{1}$ Here $\mathcal{P}$ stands for the Leray projector on solenoidal vector fields and is defined by $\mathcal{P} \stackrel{\text { def }}{=} I-\mathcal{Q}$ with $\mathcal{Q} \stackrel{\text { def }}{=} \Delta^{-1} \nabla \operatorname{div}$. 
space case and the periodic case is that in the former case one can utilize the dispersion of sound waves to get strong convergence results whereas in the latter case, the sound waves will oscillate forever, leading only to weak convergence.

In the framework of strong periodic solutions, several recent works have to be mentioned. For smooth initial data and no external force, it has been stated in [17] that slightly compressible two-dimensional solutions exist for all time. The proof is based on the exponential decay of the solutions to two-dimensional periodic incompressible Navier-Stokes equations and is unlikely to extend in higher dimension. In [15], I. Gallagher used a semi-group method to investigate the $N$-dimensional case. For sufficiently smooth data with a small incompressible part, she shows that the life span of the slightly compressible solution tends to infinity when $\varepsilon$ goes to zero. She obtained besides a convergence result for the solution "filtered" by the semi-group of a wave operator. Let us also mention a work by P. Fabrie and C. Galusinski on a simplified model (see [13]).

From now on, we shall focus on the whole space case. The periodic case is treated in [9]. For the sake of simplicity, we shall also assume that the data $\left(b_{0}^{\varepsilon}, u_{0}^{\varepsilon}, f^{\varepsilon}\right)$ do not depend on $\varepsilon$ and will be merely denoted by $\left(b_{0}, u_{0}, f\right)$ (therefore $v_{0}=\mathcal{P} u_{0}$ and $g=\mathcal{P} f$ ). This latter assumption is not essential but yields more concise statements for the convergence results.

If we adopt the framework of homogeneous Sobolev spaces and restrict ourselves to the case of initial data $\left(b_{0}, u_{0}\right) \in \dot{H}^{s} \times \dot{H}^{s^{\prime}}$, an interesting question is to find the lowest values of $s$ and $s^{\prime}$ for which local or global well-posedness may be proved. In other words, we aim at getting results in spaces which are critical in a certain sense. In view of the celebrated work by H. Fujita and T. Kato for the incompressible model (NSI) (see [14]), one can guess that the critical space for the velocity is $\dot{H}^{N / 2-1}$. Indeed, this is a critical space for (NSI). One has to recall here that this fact is closely linked to the invariance of (NSI) (for all $\ell>0$ ) by

$$
\left(v_{0}(x), f(t, x)\right) \rightarrow\left(\ell v_{0}(\ell x), \ell^{3} f\left(\ell^{2} t, \ell x\right)\right), \quad v(t, x) \rightarrow \ell v\left(\ell^{2} t, \ell x\right)
$$

and that the norm in $\dot{H}^{N / 2-1}$ is invariant by the transformation $v_{0}(x) \rightarrow \ell v_{0}(\ell x)$.

Therefore, investigating the invariance properties (if any) of $\left(N S C^{\varepsilon}\right)$ should help us to find which space may be critical. Obviously, up to a change of the pressure law $P$ into $\ell^{2} P$, system $\left(N S C^{\varepsilon}\right)$ is invariant under the transformation

$$
\begin{aligned}
& \left(\rho_{0}(x), u_{0}(x), f(t, x)\right) \rightarrow\left(\rho_{0}(\ell x), \ell u_{0}(\ell x), \ell^{3} f\left(\ell^{2} t, \ell x\right)\right), \\
& (\rho(t, x), u(t, x)) \rightarrow\left(\rho\left(\ell^{2} t, \ell x\right), \ell u\left(\ell^{2} t, \ell x\right)\right) .
\end{aligned}
$$

If we forget a while about this (first order) pressure term, we are led to consider initial data $\left(b_{0}, u_{0}\right)$ in $\dot{H}^{N / 2} \times\left(\dot{H}^{N / 2-1}\right)^{N}$. Since $\dot{H}^{N / 2}$ is not a subalgebra of $L^{\infty}$, we shall actually use a slightly smaller space, the homogeneous Besov space $B_{2,1}^{N / 2} \times\left(B_{2,1}^{N / 2-1}\right)^{N}$ (see the definition in Section 1) which is also critical according to (2). Now, $B_{2,1}^{N / 2}$ is a subalgebra of $L^{\infty}$.

In $[7,10]$, we showed that $\left(N S C^{1}\right)$ (and, more generally, the system of non-barotropic heatconducting gases) is well-posed for initial data $\left(b_{0}, u_{0}\right) \in B_{2,1}^{N / 2} \times\left(B_{2,1}^{N / 2-1}\right)^{N}$.

Let us give a rough idea of what we proved there:

- Assuming that $\left(b_{0}, u_{0}\right) \in B_{2,1}^{N / 2} \times\left(B_{2,1}^{N / 2-1}\right)^{N}$ and that $\left\|b_{0}\right\|_{B_{2,1}^{N / 2}}$ is small, we get local existence and uniqueness of a solution.

- If moreover $\left(b_{0}, u_{0}\right) \in B_{2,1}^{N / 2+\alpha} \times\left(B_{2,1}^{N / 2+\alpha-1}\right)^{N}$ for some $\alpha>0$, and $\rho_{0}$ is bounded away from zero, local existence and uniqueness holds with no smallness condition on $b_{0}$.

For small initial data, global results are expected. However, we have to pay for the omitted pressure term in (2). Nevertheless, if we assume that, in addition, $b_{0} \in B_{2,1}^{N / 2-1}$ (an assumption 
which concerns the low frequencies of $b_{0}$ only and does not change the required local regularity) then global existence in the small holds true. In $[6,10]$, we proved:

THEOREM 0.1. - There exist two positive constants

$$
c=c(\lambda, \mu, N, P) \quad \text { and } \quad M=M(\lambda, \mu, N, P)
$$

such that for all $\left(b_{0}, u_{0}, f\right)$ with $b_{0} \in B_{2,1}^{N / 2} \cap B_{2,1}^{N / 2-1}, u_{0} \in B_{2,1}^{N / 2-1}, f \in L^{1}\left(\mathbb{R}^{+} ; B_{2,1}^{N / 2-1}\right)$ and

$$
\left\|b_{0}\right\|_{B_{2,1}^{N / 2-1}}+\nu\left\|b_{0}\right\|_{B_{2,1}^{N / 2}}+\left\|u_{0}\right\|_{B_{2,1}^{N / 2-1}}+\|f\|_{L^{1}\left(B_{2,1}^{N / 2-1}\right)} \leqslant c
$$

then system $(1)($ with $\varepsilon=1)$ has a unique global solution $(b, u) \in E_{\nu}^{N / 2}$ with moreover

$$
\|(b, u)\|_{E_{\nu}^{N / 2}} \leqslant M\left(\left\|b_{0}\right\|_{B_{2,1}^{N / 2-1}}+\nu\left\|b_{0}\right\|_{B_{2,1}^{N / 2}}+\left\|u_{0}\right\|_{B_{2,1}^{N / 2-1}}+\|f\|_{L^{1}\left(B_{2,1}^{N / 2-1}\right)}\right) .
$$

If in addition, $b_{0} \in B_{2,1}^{s}, u_{0} \in B_{2,1}^{s-1}$ and $f \in L^{1}\left(\mathbb{R}^{+} ; B_{2,1}^{s-1}\right)$ for a $\left.s \in\right] N / 2, N / 2+1$, then (1) has a unique global solution $(b, u) \in E_{\nu}^{N / 2} \cap E_{\nu}^{s}$.

In the above statement, $E_{\nu}^{s}$ stands for a subspace of

$$
\left(L^{2}\left(\mathbb{R}^{+} ; B_{2,1}^{s}\right) \cap C_{b}\left(\mathbb{R}^{+} ; B_{2,1}^{s} \cap B_{2,1}^{s-1}\right)\right) \times\left(L^{1}\left(\mathbb{R}^{+} ; B_{2,1}^{s+1}\right) \cap C_{b}\left(\mathbb{R}^{+} ; B_{2,1}^{s-1}\right)\right)^{N} .
$$

The reader is referred to Definition 2.3 below for more details.

In the present paper, we address the question of global convergence to (NSI) in the critical functional setting described above.

Let us introduce a few notations: for $s \in \mathbb{R}$ and $T>0$, we denote

$$
F_{T}^{s} \stackrel{\text { def }}{=}\left(L^{1}\left(0, T ; B_{2,1}^{s+1}\right) \cap C\left([0, T] ; B_{2,1}^{s-1}\right)\right)^{N} \quad \text { and } \quad\|v\|_{F_{T}^{s}}=\|v\|_{L_{T}^{\infty}\left(B_{2,1}^{s-1}\right)}+\mu\|v\|_{L_{T}^{\infty}\left(B_{2,1}^{s+1}\right)} .
$$

We shall also use $F^{s} \stackrel{\text { def }}{=}\left(L^{1}\left(\mathbb{R}^{+} ; B_{2,1}^{s+1}\right) \cap C_{b}\left(\mathbb{R}^{+} ; B_{2,1}^{s-1}\right)\right)^{N}$ with $\|\cdot\|_{F^{s}}$ as above.

We can now state our global convergence theorem for small data:

THEOREM 0.2. - Assume $N=2,3$. There is a positive constant $\eta=\eta(\lambda, \mu, P)$ such that if $\rho_{0}^{\varepsilon}=1+\varepsilon b_{0}$ with $b_{0} \in B_{2,1}^{N / 2-1} \cap B_{2,1}^{N / 2}, u_{0} \in B_{2,1}^{N / 2-1}, f \in L^{1}\left(\mathbb{R}^{+} ; B_{2,1}^{N / 2-1}\right)$ and

$$
\left\|b_{0}\right\|_{B_{2,1}^{N / 2-1}}+\varepsilon \nu\left\|b_{0}\right\|_{B_{2,1}^{N / 2}}+\left\|u_{0}\right\|_{B_{2,1}^{N / 2-1}}+\|f\|_{L_{T}^{1}\left(B_{2,1}^{N / 2-1}\right)} \leqslant \eta
$$

for $0<\varepsilon \leqslant \varepsilon_{0}$, then the following results hold:

1. Existence: For all $\left.\varepsilon \in] 0, \varepsilon_{0}\right]$, system $\left(N S C^{\varepsilon}\right)$ has a unique global solution $\left(\rho^{\varepsilon}, u^{\varepsilon}\right)$ such that $\left(b^{\varepsilon}, u^{\varepsilon}\right)$ is uniformly bounded in $E_{\varepsilon \nu}^{N / 2}$. System (NSI) has a unique solution $v \in F^{N / 2}$.

2. Convergence: Denote $\Lambda^{s} \stackrel{\text { def }}{=} \sqrt{-\Delta}^{s / 2}$.

- For any $\alpha \in[0,1 / 6], \Lambda^{-1-\alpha}\left(\mathcal{P} u^{\varepsilon}-v\right)$ tends to zero in the set $C_{b}\left(\mathbb{R}^{+} \times \mathbb{R}^{N}\right)$ of continuous and bounded functions on $\mathbb{R}^{+} \times \mathbb{R}^{N}$. If $\alpha$ is not zero then $\Lambda^{1-\alpha}\left(\mathcal{P} u^{\varepsilon}-v\right)$ tends to zero in $L^{1}\left(\mathbb{R}^{+} ; L^{\infty}\right)$.

- For any $\alpha \in] 0,1 / 6], \Lambda^{-\alpha} \mathcal{Q} u^{\varepsilon}$ tends to zero in $L^{2}\left(\mathbb{R}^{+} ; L^{\infty}\right)$.

- If $N=3$ and $2<p<+\infty$ then $\Lambda^{1 / p-1} b^{\varepsilon}$ tends to zero in $L^{p}\left(\mathbb{R}^{+} ; L^{\infty}\right)$. If $N=2$, then $\Lambda^{-5 / 6} b^{\varepsilon}$ tends to zero in $L^{6}\left(\mathbb{R}^{+} ; L^{\infty}\right)$. 
A similar statement holds for $N \geqslant 4$ and the speed of convergence may be given in terms of power of $\varepsilon$ (see the general statement in Theorem 2.4). Let us emphasize that our convergence theorem applies to some discontinuous initial velocities and that the smallness condition is fulfilled by some velocities with large modulus provided they have enough oscillations. The reader may check that for any smooth cut-off function $\chi$ and suitably small constant $c>0$, the function $u_{0}^{\ell}(x) \stackrel{\text { def }}{=} c \ell \chi(x) \cos (\ell x)$ satisfies $\left\|u_{0}^{\ell}\right\|_{B_{2,1}^{N / 2-1}} \leqslant \eta$ for $\ell \geqslant 1$.

On one hand, Theorem 0.2 is optimal since it deals with data in critical spaces. On the other hand, it cannot be applied to large data. Considering that in many cases the solution of the limit system (NSI) may have a very long life span $T$, possibly infinite, even though the initial data are large (e.g., case $N=2$ or $N=3$ axisymmetric), it seems natural that the corresponding solution for $\left(N S C^{\varepsilon}\right)$ should also have a large life span $T_{\varepsilon}$ for small $\varepsilon$. Referring to some recent works in the periodic case (see [15] and [17]) we expect a result such that $\lim \inf _{\varepsilon \rightarrow 0} T_{\varepsilon} \geqslant T$ to be true.

To achieve this in the whole space case, it actually suffices to consider slightly more regular data. In addition, we obtain global existence for small $\varepsilon$ provided that the corresponding incompressible solution is global. More precisely, we have

THEOREM 0.3. - Suppose that $N=3$ and that, for a $\alpha \in] 0,1 / 2[$, we have

$$
b_{0} \in B_{2,1}^{1 / 2} \cap B_{2,1}^{3 / 2+\alpha}, \quad u_{0} \in B_{2,1}^{1 / 2} \cap B_{2,1}^{1 / 2+\alpha} \quad \text { and } f \in L^{1}\left(\mathbb{R}^{+} ; B_{2,1}^{1 / 2} \cap B_{2,1}^{1 / 2+\alpha}\right) .
$$

Suppose that the incompressible system $(N S I)$ with initial datum $\mathcal{P} u_{0}$ and external force $\mathcal{P} f$ has a solution $v \in F_{T_{0}}^{3 / 2} \cap F_{T_{0}}^{3 / 2+\alpha}$ for a positive $T_{0}$ possibly infinite.

Then there exists a positive $\varepsilon_{0}$ depending on the initial data, on the incompressible solution $v$, on the pressure law $P$, and on $\lambda, \mu$, and $\alpha$, and such that for all $0<\varepsilon \leqslant \varepsilon_{0}$, system (1) has a unique solution $\left(b^{\varepsilon}, u^{\varepsilon}\right)$ in $E_{\varepsilon \nu, T_{0}}^{3 / 2} \cap E_{\varepsilon \nu, T_{0}}^{3 / 2+\alpha}$.

Moreover, $\mathcal{P} u_{\varepsilon}$ tends to $v$ in $F_{T_{0}}^{3 / 2} \cap F_{T_{0}}^{3 / 2+\alpha}$ and $\left(\Lambda^{\alpha-1+1 / p} b^{\varepsilon}, \Lambda^{\alpha-1+1 / p} \mathcal{Q} u^{\varepsilon}\right)$ tends to 0 in every $L^{p}\left(0, T_{0} ; L^{\infty}\right)$ such that $2<p<+\infty$.

In the statement above, the notation $E_{\kappa, T}^{s}$ stands for a subspace of

$$
\left(L^{2}\left([0, T] ; B_{2,1}^{s}\right) \cap C\left([0, T] ; B_{2,1}^{s} \cap B_{2,1}^{s-1}\right)\right) \times\left(L^{1}\left([0, T] ; B_{2,1}^{s+1}\right) \cap C\left([0, T] ; B_{2,1}^{s-1}\right)\right)^{N}
$$

(see the details in Definition 2.3 below).

In the case $N=2$ and under the assumptions made in the theorem above (even if $\alpha=0$ in fact), the incompressible solution is always global and we obtain the following result (to be compared with the corresponding one proved in the periodic setting in [17]).

THEOREM 0.4. - Let

$$
\alpha \in] 0,1 / 6], \quad b_{0} \in B_{2,1}^{0} \cap B_{2,1}^{1+\alpha}, \quad u_{0} \in B_{2,1}^{0} \cap B_{2,1}^{\alpha} \quad \text { and } \quad f \in L^{1}\left(\mathbb{R}^{+} ; B_{2,1}^{0} \cap B_{2,1}^{\alpha}\right) .
$$

Then the incompressible system (NSI) with initial datum $\mathcal{P} u_{0}$ and external force $\mathcal{P} f$ has a global solution $v \in F^{1} \cap F^{1+\alpha}$.

Moreover there exists a positive $\varepsilon_{0}$ depending only on the initial data, on $P$, and on the parameters $\lambda, \mu, \alpha$, and such that for all $0<\varepsilon \leqslant \varepsilon_{0}$, system (1) has a unique global solution $\left(b^{\varepsilon}, u^{\varepsilon}\right)$ in $E_{\varepsilon \nu}^{1} \cap E_{\varepsilon \nu}^{1+\alpha}$. The incompressible part $\mathcal{P} u_{\varepsilon}$ tends to $v$ in $F^{1} \cap F^{1+\alpha}$ and $\left(\Lambda^{\alpha-3 / 4} b^{\varepsilon}, \Lambda^{\alpha-3 / 4} \mathcal{Q} u_{\varepsilon}\right)$ tends to 0 in $L^{4}\left(0,+\infty ; L^{\infty}\right)$.

Remark 0.5. - Whether a similar result holds true in critical spaces (that is for $\alpha=0$ ) is opened. The need of additional regularity appears in many points of the proof because it provides some decay in $\varepsilon$. 
Remark 0.6. - Despite the additional regularity assumption, the statements above do hold for a large class of discontinuous initial velocities. Let us mention that all the results which pertain to supercritical initial data hold in the Sobolev spaces framework as well. We kept the Besov spaces for the sake of unity.

Remark 0.7. - Similar results are very likely to hold for the system of heat conductive gases considered in [8]. No additional mathematical difficulties are expected: the arguments used in the present paper probably suffice but the computations to be done are certainly worse.

Remark 0.8. - For the sake of simplicity, we supposed that the data $b_{0}, u_{0}$ and $f$ were independent of $\varepsilon$. It goes without saying that convergence results in the same spirit may be stated for data depending on $\varepsilon$ provided that $\mathcal{P} u_{0}^{\varepsilon}$ and $\mathcal{P} f^{\varepsilon}$ converge strongly in appropriate spaces, and that $\mathcal{Q} u_{0}^{\varepsilon}, \mathcal{Q} f^{\varepsilon}$ and $b_{0}^{\varepsilon}$ are uniformly bounded for small $\varepsilon$.

Our paper is organized as follows. In the first section, we define some functional spaces (homogeneous and hybrid Besov spaces), recall some basic tools in paradifferential calculus and state some tame estimates for the composition or the product. In Section 2, we give the general statements of existence and convergence for $\left(N S C^{\varepsilon}\right)$ (from which Theorems 0.2, 0.3 and 0.4 easily stem). We also give the outlines of the proof to help the reader to make his way through the technicalities of the following sections. Section 3 is devoted to the proof of the convergence result in the small. In Section 4, we prove estimates for the paralinearisation of $\left(N S C^{\varepsilon}\right)$. These estimates combined with dispersive inequalities for the linear wave equation, will be at the root of the proof of our existence and convergence result in the case of large data (Section 5). For the sake of completeness, we put in Section 6 some regularity results on incompressible NavierStokes equations that we did not manage to find in the huge literature devoted to the subject. Some technical lemmas have been postponed in an appendix.

Notation. - Throughout the paper, $C$ stands for a "harmless constant" which never depends on $\varepsilon$, and we sometimes use the notation $A \lesssim B$ as an equivalent to $A \leqslant C B$. The notation $A \approx B$ means that $A \lesssim B$ and $B \lesssim A$.

\section{Homogeneous and hybrid Besov spaces}

Let us first recall the definition and some basic properties of homogeneous Besov spaces. They may be defined through the use of a dyadic partition of unity in Fourier variables called homogeneous Littlewood-Paley decomposition. For that purpose, choose a $\varphi \in C^{\infty}\left(\mathbb{R}^{N}\right)$ supported in, say, $\mathcal{C} \stackrel{\text { def }}{=}\left\{\xi \in \mathbb{R}^{N}, 5 / 6 \leqslant|\xi| \leqslant 12 / 5\right\}$ and such that

$$
\sum_{q \in \mathbb{Z}} \varphi\left(2^{-q} \xi\right)=1 \quad \text { if } \xi \neq 0
$$

Denoting $h=\mathcal{F}^{-1} \varphi$, we then define the dyadic blocks as follows

$$
\Delta_{q} u \stackrel{\text { def }}{=} \varphi\left(2^{-q} D\right) u=2^{q N} \int_{\mathbb{R}^{N}} h\left(2^{q} y\right) u(x-y) \mathrm{d} y, \quad \text { and } \quad S_{q} u=\sum_{k \leqslant q-1} \Delta_{k} u .
$$

The formal decomposition

$$
u=\sum_{q \in \mathbb{Z}} \Delta_{q} u
$$

is called homogeneous Littlewood-Paley decomposition.

$4^{\mathrm{e}}$ SÉRIE - TOME $35-2002-\mathrm{N}^{\circ} 1$ 
The equality (3) holds modulo polynomials: if $u \in \mathcal{S}^{\prime}\left(\mathbb{R}^{N}\right)$, then $\sum_{q \in \mathbb{Z}} \Delta_{q} u$ converges modulo $\mathcal{P}\left[\mathbb{R}^{N}\right]$ and (3) holds in $\mathcal{S}^{\prime}\left(\mathbb{R}^{N}\right) / \mathcal{P}\left[\mathbb{R}^{N}\right]$ (see [29]). Furthermore, it has nice properties of quasi-orthogonality: with our choice of $\varphi$, we have

$$
\Delta_{k} \Delta_{q} u \equiv 0 \quad \text { if }|k-q| \geqslant 2 \quad \text { and } \quad \Delta_{k}\left(S_{q-1} u \Delta_{q} u\right) \equiv 0 \quad \text { if }|k-q| \geqslant 4 .
$$

It is easy to check that

$$
\|u\|_{\dot{H}^{s}} \approx\|u\|_{B_{2,2}^{s}} \stackrel{\text { def }}{=}\left(\sum_{q \in \mathbb{Z}} 2^{2 q s}\left\|\Delta_{q} u\right\|_{L^{2}}^{2}\right)^{1 / 2}
$$

More generally, we shall use the following notation for $s \in \mathbb{R}, r \in[1,+\infty[, p \in[1,+\infty]$ and $u \in \mathcal{S}^{\prime}\left(\mathbb{R}^{N}\right)$, we set

$$
\|u\|_{B_{p, r}^{s}} \stackrel{\text { def }}{=}\left(\sum_{q \in \mathbb{Z}}\left(2^{s q}\left\|\Delta_{q} u\right\|_{L^{p}}\right)^{r}\right)^{1 / r} \text { and }\|u\|_{B_{p, \infty}^{s}} \stackrel{\text { def }}{=} \sup _{q \in \mathbb{Z}} 2^{s q}\left\|\Delta_{q} u\right\|_{L^{p}} .
$$

We shall adopt the following definition for homogeneous Besov spaces with the third index equal to one:

Definition 1.1. - Let $s \in \mathbb{R}$, and $p \in[1,+\infty]$. Denote $m=-[N / p+1-s]$. If $m<0$, then we define $B_{p, 1}^{s}\left(\mathbb{R}^{N}\right)$ as

$$
B_{p, 1}^{s}=\left\{u \in \mathcal{S}^{\prime}\left(\mathbb{R}^{N}\right),\|u\|_{B_{p, 1}^{s}}<\infty \text { and } u=\sum_{q \in \mathbb{Z}} \Delta_{q} u \text { in } \mathcal{S}^{\prime}\left(\mathbb{R}^{N}\right)\right\} .
$$

If $m \geqslant 0$, we denote by $\mathcal{P}_{m}\left[\mathbb{R}^{N}\right]$ the set of polynomials of degree $\leqslant m$ and we set

$$
B_{p, 1}^{s}=\left\{u \in \mathcal{S}^{\prime}\left(\mathbb{R}^{N}\right) / \mathcal{P}_{m}\left[\mathbb{R}^{N}\right],\|u\|_{B_{p, 1}^{s}}<\infty \text { and } u=\sum_{q \in \mathbb{Z}} \Delta_{q} u \text { in } \mathcal{S}^{\prime}\left(\mathbb{R}^{N}\right) / \mathcal{P}_{m}\left[\mathbb{R}^{N}\right]\right\}
$$

Remark. - From the above definition, it is not hard to check that $B_{2,1}^{s} \hookrightarrow \dot{H}^{s}$ (where $\hookrightarrow$ means continuous embedding) but that these two spaces are very close anyway.

From now on, the notation $B_{p}^{s}$ will stand for $B_{p, 1}^{s}$. In the case where $u$ is valued in $\mathbb{R}^{m}$, the notation $\|u\|_{B_{p}^{s}}$ will stand for $\sum_{i}\left\|u^{i}\right\|_{B_{p}^{s}}$.

The following result (which shows amongst other that the definition of $B_{p}^{s}$ is independent of the choice of $\varphi$ ) is very useful:

Lemma 1.2. - Let $u \in B_{p}^{s}$ and $\psi \in C_{0}^{\infty}\left(\mathbb{R}^{N}\right)$ supported in the annulus $C\left(0, R_{1}, R_{2}\right)$. Then there exists a sequence $\left(c_{q}\right)_{q \in \mathbb{Z}}$ such that $\sum_{q} c_{q} \leqslant 1$ and

$$
\left\|\psi\left(2^{-q} D\right) u\right\|_{L^{p}} \lesssim c_{q} 2^{-q s}\|u\|_{B_{p}^{s}} \quad \text { for all } q \in \mathbb{Z} .
$$

Conversely, suppose that $u=\sum_{q} u_{q}$ in $\mathcal{S}^{\prime}\left(\mathbb{R}^{N}\right)\left(\right.$ or in $\mathcal{S}^{\prime}\left(\mathbb{R}^{N}\right) / \mathcal{P}_{m}\left[\mathbb{R}^{N}\right]$ if $m \stackrel{\text { def }}{=}-[N / p+1-s]$ is nonnegative) with $\operatorname{Supp} \widehat{u}_{q} \subset 2^{q} C\left(0, R_{1}, R_{2}\right)$, and that 


$$
\sum_{q \in \mathbb{Z}} 2^{q s}\left\|u_{q}\right\|_{L^{p}}=K<+\infty
$$

Then $u \in B_{p}^{s}$ and $\|u\|_{B_{p}^{s}} \lesssim K$.

Let us now state some classical properties for these Besov spaces, the proof of which may be found in [29] or [30].

PROPOSITION 1.3. - The following properties hold:

(i) Density: if $p<+\infty$ and $|s| \leqslant N / p$ then $C_{0}^{\infty}$ is dense in $B_{p}^{s}$.

(ii) Derivation: there exists a universal constant $C$ such that

$$
C^{-1}\|u\|_{B_{p}^{s}} \leqslant\|\nabla u\|_{B_{p}^{s-1}} \leqslant C\|u\|_{B_{p}^{s}} .
$$

(ii)' Fractional derivation: let $\Lambda \stackrel{\text { def }}{=} \sqrt{-\Delta}$ and $\sigma \in \mathbb{R}$. Then the operator $\Lambda^{\sigma}$ is an isomorphism from $B_{p}^{s}$ to $B_{p}^{s-\sigma}$.

(iii) Sobolev embeddings: if $p_{1}<p_{2}$ then $B_{p_{1}}^{s} \hookrightarrow B_{p_{2}}^{s-N\left(1 / p_{1}-1 / p_{2}\right)}$.

(iv) Algebraic properties: for $s>0, B_{p}^{s} \cap L^{\infty}$ is an algebra.

(v) Interpolation: $\left[B_{p}^{s_{1}}, B_{p}^{s_{2}}\right]_{\theta}=B_{p}^{\theta s_{1}+(1-\theta) s_{2}}$.

(vi) Scaling properties: for all $\lambda>0$ and $u \in B_{p}^{s}$, we have

$$
\|u(\lambda \cdot)\|_{B_{p}^{s}} \approx \lambda^{s-N / p}\|u\|_{B_{p}^{s}} .
$$

We will make an extensive use of the space $B_{p}^{N / p}$ which is a subalgebra of the set $C_{0}$ of continuous functions vanishing at infinity.

Let us state some continuity results for the product (see [30]).

PROPOSITION 1.4. - If $u \in B_{p_{1}}^{s_{1}}$ and $v \in B_{p_{2}}^{s_{2}}$ with $1 \leqslant p_{1} \leqslant p_{2} \leqslant+\infty, s_{1} \leqslant N / p_{1}$, $s_{2} \leqslant N / p_{2}$ and $s_{1}+s_{2}>0$ then $u v \in B_{p_{2}}^{s_{1}+s_{2}-N / p_{1}}$ and

$$
\|u v\|_{B_{p_{2}}^{s_{1}+s_{2}-N / p_{1}}} \lesssim\|u\|_{B_{p_{1}}^{s_{1}}}\|v\|_{B_{p_{2}}^{s_{2}}} .
$$

If $u \in B_{p_{1}}^{s_{1}} \cap B_{p_{2}}^{s_{2}}$ and $v \in B_{p_{1}}^{t_{1}} \cap B_{p_{2}}^{t_{2}}$ with $1 \leqslant p_{1}, p_{2} \leqslant+\infty, s_{1}, t_{1} \leqslant N / p_{1}$ and $s_{1}+t_{2}=s_{2}+t_{1}>N_{\max }\left(0, \frac{1}{p_{1}}+\frac{1}{p_{2}}-1\right)$ then $u v \in B_{p_{2}}^{s_{1}+t_{2}-N / p_{1}}$ and

$$
\|u v\|_{B_{p_{2}}^{s_{1}+t_{2}-N / p_{1}}} \lesssim\|u\|_{B_{p_{1}}^{s_{1}}}\|v\|_{B_{p_{2}}^{t_{2}}}+\|u\|_{B_{p_{2}}^{s_{2}}}\|v\|_{B_{p_{1}}^{t_{1}}}
$$

Moreover, if $s_{1}=0$ and $p_{1}=+\infty$ then $\|u\|_{B_{\infty}^{0}}$ may be replaced with $\|u\|_{L^{\infty}}$.

We finally need a composition lemma in $B_{p}^{s}$ (see [30]).

LEMma 1.5. - Let $s>0, p \in[1,+\infty]$ and $u \in B_{p}^{s} \cap L^{\infty}$. Let $F \in W_{\mathrm{loc}}^{[s]+2, \infty}\left(\mathbb{R}^{N}\right)$ such that $F(0)=0$. Then $F(u) \in B_{p}^{s}$ and there exists a constant $C=C\left(s, p, N, F,\|u\|_{L^{\infty}}\right)$ such that

$$
\|F(u)\|_{B_{p}^{s}} \leqslant C\|u\|_{B_{p}^{s}} .
$$

Notation. - For any Banach space $X, 0<T \leqslant+\infty$ and $1 \leqslant r \leqslant+\infty$, we shall denote by $L^{r}(0, T ; X)$ the set of measurable functions on $] 0, T[$ valued in $X$ and such that the map $t \mapsto\|u(t)\|_{X}$ belongs to the Lebesgue space $L^{r}(0, T)$. In the case $T=+\infty$, we shall sometimes 
denote the space above by $L^{r}(X)$, and by $\|\cdot\|_{L^{r}(X)}$ the associated norm. By $C([0, T] ; X)$ (resp. $C([0,+\infty] ; X)$ or $\left.C_{b}\left(\mathbb{R}^{+} ; X\right)\right)$ we mean the subset of functions $u$ of $L^{\infty}(0, T ; X)$ such that the map $t \mapsto u(t, \cdot)$ is continuous from $[0, T]$ (resp. $\mathbb{R}^{+}$) to $X$.

Remark 1.6. - For $u=u(t, x)$ in $L^{r}\left(0, T ; B_{p}^{s}\right)$, we have the following scaling property:

$$
\left\|u\left(\lambda^{a} \cdot \lambda^{b} \cdot\right)\right\|_{L_{T}^{r}\left(B_{p}^{s}\right)} \approx \lambda^{b(s-N / p)-a / r}\|u\|_{L_{\lambda^{a} T}^{r}\left(B_{p}^{s}\right)} .
$$

Owing to the fact that the change of variables of Definition 2 does not really leave system (1) invariant, the use of homogeneous spaces is not quite appropriate. For that reason, we shall introduce some hybrid Besov spaces where the growth conditions satisfied by the dyadic blocks are different for low and high frequencies. These very same spaces have been used in [6]. We here recall their definition

Definition 1.7. - Let $s \in \mathbb{R}, \alpha>0$ and $1 \leqslant r \leqslant+\infty$. We set

$$
\|u\|_{\widetilde{B}_{\alpha}^{s, r}} \stackrel{\text { def }}{=} \sum_{q \in \mathbb{Z}} 2^{q s} \max \left(\alpha, 2^{-q}\right)^{1-2 / r}\left\|\Delta_{q} u\right\|_{L^{2}} .
$$

Let $m=-[N / 2+2-2 / r-s]$. We then define

$$
\begin{gathered}
\widetilde{B}_{\alpha}^{s, r}\left(\mathbb{R}^{N}\right)=\left\{u \in \mathcal{S}^{\prime}\left(\mathbb{R}^{N}\right),\|u\|_{\widetilde{B}_{\alpha}^{s, r}}<+\infty\right\} \quad \text { if } m<0, \\
\widetilde{B}_{\alpha}^{s, r}\left(\mathbb{R}^{N}\right)=\left\{u \in \mathcal{S}^{\prime}\left(\mathbb{R}^{N}\right) / \mathcal{P}_{m}\left(\mathbb{R}^{N}\right),\|u\|_{\widetilde{B}_{\alpha}^{s, r}}<+\infty\right\} \quad \text { if } m \geqslant 0 .
\end{gathered}
$$

Notation. - We will often use the following notation:

$$
u_{B F} \stackrel{\text { def }}{=} \sum_{q \leqslant\left[-\log _{2} \alpha\right]} \Delta_{q} u \text { and } u_{H F} \stackrel{\text { def }}{=} \sum_{q>\left[-\log _{2} \alpha\right]} \Delta_{q} u .
$$

Remark 1.8. - (i) We have $\widetilde{B}_{\alpha}^{s, 2}=B_{2}^{s}$.

(ii) If $r \geqslant 2$ then $\widetilde{B}_{\alpha}^{s, r}=B_{2}^{s+2 / r-1} \cap B_{2}^{s}$ and

$$
\|u\|_{\widetilde{B}_{\alpha}^{s, r}} \approx\|u\|_{B_{2}^{s+2 / r-1}}+\alpha^{1-2 / r}\|u\|_{B_{2}^{s}} .
$$

If $r \leqslant 2$ then $\widetilde{B}_{\alpha}^{s, r}=B_{2}^{s+2 / r-1}+B_{2}^{s}$ and

$$
\|u\|_{\widetilde{B}_{\alpha}^{s, r}} \approx\left\|u_{B F}\right\|_{B_{2}^{s+2 / r-1}}+\alpha^{1-2 / r}\left\|u_{H F}\right\|_{B_{2}^{s}} .
$$

(iii) For all $\lambda>0$ and $u \in \widetilde{B}_{\alpha}^{s, r}$, we have

$$
\|u(\lambda \cdot)\|_{\widetilde{B}_{\alpha}^{s, r}} \approx \lambda^{s-N / 2+2 / r-1}\|u\|_{\widetilde{B}_{\lambda \alpha}^{s, r}}
$$

Throughout the paper, we shall use some smatterings of paradifferential calculus: the paraproduct introduced by J.-M. Bony in [1]. This is a convenient way to define a generalized product between distributions which is continuous in many functional spaces where the usual 
product does not make sense. The paraproduct between $u$ and $v$ is given by

$$
T_{u} v \stackrel{\text { def }}{=} \sum_{q \in \mathbb{Z}} S_{q-1} u \Delta_{q} v
$$

We have the following Bony decomposition (modulo a polynomial):

$$
u v=T_{u} v+T_{v} u+R(u, v) \quad \text { with } R(u, v) \stackrel{\text { def }}{=} \sum_{q \in \mathbb{Z}} \Delta_{q} u \widetilde{\Delta}_{q} v \text { and } \widetilde{\Delta}_{q}=\Delta_{q-1}+\Delta_{q}+\Delta_{q+1} .
$$

The notation $T_{u}^{\prime} v \stackrel{\text { def }}{=} T_{u} v+R(u, v)$ will be employed likewise.

In the following proposition, we state some continuity properties for the remainder $R$ and paraproduct $T$ in Besov spaces (see [30] for the proof).

Proposition 1.9. - Let $1 \leqslant p_{1}, p_{2} \leqslant+\infty$. For all $s_{2} \in \mathbb{R}$ and $s_{1} \leqslant N / p_{1}$, we have

$$
\left\|T_{u} v\right\|_{B_{p_{2}}^{s_{1}+s_{2}}} \lesssim\|u\|_{B_{\infty, \infty}^{s_{1}}}\|v\|_{B_{p_{2}}^{s_{2}}}
$$

If $s_{1}=0$, the above inequality holds with $\|u\|_{L^{\infty}}$ instead of $\|u\|_{B_{\infty, \infty}^{s}}$.

If $\left(s_{1}, s_{2}\right) \in \mathbb{R}^{2}$ satisfies $s_{1}+s_{2}>N_{\max }\left(0, \frac{1}{p_{1}}+\frac{1}{p_{2}}-1\right)$ then

$$
\|R(u, v)\|_{B_{p_{2}}^{s_{1}+s_{2}-N / p_{1}}} \lesssim\|u\|_{B_{p_{1}, \infty}^{s_{1}}}\|v\|_{B_{p_{2}}^{s_{2}}}
$$

Now, estimates of Proposition 1.4 obviously stem from Proposition 1.9.

\section{Main results and sketch of the proof}

\subsection{The linearized system}

Let us split the velocity into a divergence-free part $\mathcal{P} u^{\varepsilon}$ and a gradient part $\mathcal{Q} u^{\varepsilon}$ (recall that $\mathcal{P} \stackrel{\text { def }}{=} I-\nabla \Delta^{-1} \operatorname{div}$ and $\left.\mathcal{Q} \stackrel{\text { def }}{=} I-\mathcal{P}\right)$. System (1) reads

$$
\left\{\begin{array}{l}
\partial_{t} b^{\varepsilon}+\frac{\operatorname{div} \mathcal{Q} u^{\varepsilon}}{\varepsilon}=-\operatorname{div}\left(u^{\varepsilon} b^{\varepsilon}\right), \\
\partial_{t} \mathcal{Q} u^{\varepsilon}-\nu \Delta \mathcal{Q} u^{\varepsilon}+\frac{\nabla b^{\varepsilon}}{\varepsilon}=\mathcal{Q}\left(f-u^{\varepsilon} \cdot \nabla u^{\varepsilon}-\frac{\varepsilon b^{\varepsilon}}{1+\varepsilon b^{\varepsilon}} \mathcal{A} u^{\varepsilon}-K\left(\varepsilon b^{\varepsilon}\right) \frac{\nabla b^{\varepsilon}}{\varepsilon}\right), \\
\partial_{t} \mathcal{P} u^{\varepsilon}-\mu \Delta \mathcal{P} u^{\varepsilon}=\mathcal{P}\left(f-u^{\varepsilon} \cdot \nabla u^{\varepsilon}-\frac{\varepsilon b^{\varepsilon}}{1+\varepsilon b^{\varepsilon}} \mathcal{A} u^{\varepsilon}\right),
\end{array}\right.
$$

with $K(z) \stackrel{\text { def }}{=} \frac{P^{\prime}(1+z)}{1+z}-1$ (hence $\left.K(0)=0\right)$ and $\mathcal{A} \stackrel{\text { def }}{=} \mu \Delta+(\lambda+\mu) \nabla$ div.

Observe that there is no linear coupling between the last equation and the first two so that we expect standard results on heat equations (see Proposition 7.3) to yield a control on $\mathcal{P} u^{\varepsilon}$. On the other hand, there is a linear coupling between the first two equations. Since, in homogeneous spaces, estimating $\mathcal{Q} u^{\varepsilon}$ or $d^{\varepsilon} \stackrel{\text { def }}{=} \Lambda^{-1} \operatorname{div} \mathcal{Q} u^{\varepsilon}$ is equivalent, we are led to investigate carefully the following mixed linear system:

$$
\left\{\begin{array}{l}
\partial_{t} b+u \cdot \nabla b+\frac{\Lambda d}{\varepsilon}=F \\
\partial_{t} d+u \cdot \nabla d-\nu \Delta d-\frac{\Lambda b}{\varepsilon}=G .
\end{array}\right.
$$


Note that we included the convection terms $u \cdot \nabla b$ and $u \cdot \nabla d$ in the system above (see [6] for more explanations). Let us concentrate for a while on the case $u=0$. The matrix operator associated to (11) reads

$$
A(D)=\left(\begin{array}{cc}
0 & -\varepsilon^{-1} \Lambda \\
\varepsilon^{-1} \Lambda & -\nu \Lambda^{2}
\end{array}\right) .
$$

A rough study of the eigenvalues makes us expect a different behaviour of (11) for low and high frequencies. Indeed, for $\nu \varepsilon|\xi|<2$, the eigenvalues are

$$
\lambda^{ \pm}(\xi)=-\frac{\nu|\xi|^{2}}{2}\left(1 \pm i \sqrt{\frac{4}{\varepsilon^{2} \nu^{2}|\xi|^{2}}-1}\right)
$$

so that the linear operator is very similar (for $\nu \varepsilon|\xi| \ll 1$ ) to

$$
\partial_{t}-\nu \Delta / 2 \pm \mathrm{i} \Lambda / \varepsilon
$$

In contrast, for $\nu \varepsilon|\xi|>2$, we have

$$
\lambda^{ \pm}(\xi)=-\frac{\nu|\xi|^{2}}{2}\left(1 \pm \sqrt{1-\frac{4}{\varepsilon^{2} \nu^{2}|\xi|^{2}}}\right)
$$

which means that a parabolic mode and a damped mode coexist.

Further considerations on the eigenvectors motivates the use of hybrid Besov norms (see [6] for more details). Now, a straightforward change of variables in Proposition 2.3 of [6], and a use of (5), (6) and (9) yield the following result:

Proposition 2.1. - Denote

$$
V(t)=\int_{0}^{t}\|u(\tau)\|_{B_{2}^{N / 2+1}} \mathrm{~d} \tau .
$$

Then for any $1-N / 2<s \leqslant 1+N / 2$, the following estimate holds on $[0, T[$ for a constant $C=C(N, s)$ :

$$
\begin{aligned}
& \|b(t)\|_{\widetilde{B}_{\varepsilon \nu}^{s, \infty}}+\|d(t)\|_{B_{2}^{s-1}}+\nu \int_{0}^{t}\left(\|b(\tau)\|_{\widetilde{B}_{\varepsilon \nu}^{s, 1}}+\|d(\tau)\|_{B_{2}^{s+1}}\right) \mathrm{d} \tau \\
& \leqslant C \mathrm{e}^{C V(t)}\left(\left\|b_{0}\right\|_{\widetilde{B}_{\varepsilon \nu}^{s, \infty}}+\left\|d_{0}\right\|_{B_{2}^{s-1}}+\int_{0}^{t} \mathrm{e}^{-C V(\tau)}\left(\|F(\tau)\|_{\widetilde{B}_{\varepsilon \nu}^{s, \infty}}+\|G(\tau)\|_{B_{2}^{s-1}}\right) \mathrm{d} \tau\right) .
\end{aligned}
$$

The above estimate lies on an energy method. This clearly "kills" the highly oscillating properties of the low frequencies. This is quite tiresome since the "low frequencies" may be very high when $\varepsilon$ goes to zero and this is a well known fact that large oscillations may help us to pass to the limit (see for example [32]).

Obviously, there is no hope of improving (12) as far as one uses estimates in spaces which are built on $L^{2}$. This motivates the use of spaces built on $L^{p}$ for a $p>2$. We should mention here that the investigation of $L^{p}$ estimates for a system analogous to (11) (with $u=0$ ) has been 
done in [19]. The motivation of the authors however was the study of the asymptotic behaviour of smooth solutions of compressible Navier-Stokes equations. We do not know if these estimates may help us to study the slightly compressible fluids.

Following B. Desjardins and E. Grenier in [11], we shall use some dispersive inequalities for the wave equation: the so-called Strichartz estimates (see, e.g., $[31,16,20]$ and the references therein). Here is the statement that we need. The reader may refer to the appendix, Proposition 7.1 for a more general statement and a sketchy proof.

PROPOSITION 2.2. - Let $(b, d)$ be a solution of the following system

$$
\left\{\begin{array}{l}
\partial_{t} b+\varepsilon^{-1} \Lambda d=F \\
\partial_{t} d-\varepsilon^{-1} \Lambda b=G
\end{array}\right.
$$

Then for any $s \in \mathbb{R}$ and positive $T$ (possibly infinite), the following estimate holds

$$
\|(b, d)\|_{L_{T}^{r}\left(B_{p}^{s+N(1 / p-1 / 2)+1 / r}\right)} \lesssim \varepsilon^{1 / r}\left(\left\|\left(b_{0}, d_{0}\right)\right\|_{B_{2}^{s}}+\|(F, G)\|_{L_{T}^{1}\left(B_{2}^{s}\right)}\right)
$$

with $p \geqslant 2,2 / r \leqslant \min (1,(N-1)(1 / 2-1 / p))$ and $(r, p, N) \neq(2, \infty, 3)$.

Now, we can expect Proposition 2.1 combined with estimates for the heat equation to provide us with uniform estimates for $\left(b^{\varepsilon}, u^{\varepsilon}\right)$, thus uniform bounds for the right-hand side of $\left(W^{\varepsilon}\right)$. According to Proposition 2.2, this should give us some convergence result.

No further arguments are needed to get global convergence in the small in critical spaces, or local convergence in the large for more regular data. In the next two sections, we shall develop these ideas and give complete statements of our convergence results.

\subsection{Global convergence in the small}

Let us introduce the following notation:

Definition 2.3. - For $0<T \leqslant+\infty, \kappa>0$ and $s \in \mathbb{R}$, we denote

$E_{\kappa, T}^{s}=\left\{(b, u) \in\left(L^{1}\left(0, T ; \widetilde{B}_{\kappa}^{s, 1}\right) \cap C\left([0, T] ; \widetilde{B}_{\kappa}^{s, \infty}\right)\right) \times\left(L^{1}\left(0, T ; B_{2,1}^{s+1}\right) \cap C\left([0, T] ; B_{2,1}^{s-1}\right)\right)^{N}\right\}$

and $\|(b, u)\|_{E_{\kappa, T}^{s}}=\|b\|_{L_{T}^{\infty}\left(\widetilde{B}_{\kappa}^{s, \infty}\right)}+\|v\|_{L_{T}^{\infty}\left(B_{2,1}^{s-1}\right)}+\|b\|_{L_{T}^{1}\left(\widetilde{B}_{\kappa}^{s, 1}\right)}+\|v\|_{L_{T}^{1}\left(B_{2,1}^{s+1}\right)}$.

For $T=+\infty$, we will merely write $E_{\kappa}^{s}$ instead of $E_{\kappa, \infty}^{s}$.

In Section 3, we shall prove the following result:

THEOREM 2.4. - There exist two positive constants

$$
\eta=\eta(N, \lambda, \mu, P) \quad \text { and } \quad M=M(N, \lambda, \mu, P)
$$

such that if $b_{0} \in B_{2}^{N / 2-1} \cap B_{2}^{N / 2}, u_{0} \in B_{2}^{N / 2-1}, f \in L^{1}\left(\mathbb{R}^{+} ; B_{2}^{N / 2-1}\right)$ satisfy (for all $\left.0<\varepsilon \leqslant \varepsilon_{0}\right)$

$$
C_{0}^{\varepsilon \nu} \stackrel{\text { def }}{=}\left\|b_{0}\right\|_{B_{2}^{N / 2-1}}+\varepsilon \nu\left\|b_{0}\right\|_{B_{2}^{N / 2}}+\left\|u_{0}\right\|_{B_{2}^{N / 2-1}}+\|f\|_{L^{1}\left(B_{2}^{N / 2-1}\right)} \leqslant \eta
$$

then the following results hold:

$4^{\mathrm{e}}$ SÉRIE - TOME $35-2002-\mathrm{N}^{\circ} 1$ 
1. Existence: For all $0<\varepsilon \leqslant \varepsilon_{0}$, system (1) has a unique solution $\left(b^{\varepsilon}, u^{\varepsilon}\right)$ in $E_{\varepsilon \nu}^{N / 2}$ such that

$$
\left\|\left(b^{\varepsilon}, u^{\varepsilon}\right)\right\|_{E_{\varepsilon \nu}^{N / 2}} \leqslant M C_{0}^{\varepsilon \nu} .
$$

System (NSI) has a (unique) solution $v \in F^{N / 2}$ such that

$$
\|v\|_{L^{1}\left(B_{2}^{N / 2+1}\right)}+\|v\|_{L^{\infty}\left(B_{2}^{N / 2-1}\right)} \leqslant M\left(\left\|\mathcal{P} u_{0}\right\|_{B_{2}^{N / 2-1}}+\|\mathcal{P} f\|_{L^{1}\left(B_{2}^{N / 2-1}\right)}\right) .
$$

2. Convergence: For any $\alpha \in[0,1 / 2]$ if $N \geqslant 4, \alpha \in[0,1 / 2[$ if $N=3$ and $\alpha \in[0,1 / 6]$ if $N=2, \mathcal{P} u^{\varepsilon}$ tends to $v$ in $C\left([0,+\infty] ; B_{\infty}^{-1-\alpha}\right)$. Moreover,

- If $N \geqslant 4$ : For all $p \in\left[p_{N},+\infty\right]$ with $p_{N} \stackrel{\text { def }}{=} 2(N-1) /(N-3)$, we have

$$
\begin{aligned}
& \left\|\mathcal{P} u^{\varepsilon}-v\right\|_{L^{1}\left(B_{p}^{N / p+1 / 2}\right)}+\left\|\mathcal{P} u^{\varepsilon}-v\right\|_{L^{\infty}\left(B_{p}^{N / p-3 / 2}\right)}+\left\|\mathcal{Q} u^{\varepsilon}\right\|_{L^{2}\left(B_{p}^{N / p-1 / 2}\right)} \\
& \quad+\left\|b^{\varepsilon}\right\|_{L^{2}\left(B_{p}^{N / p-1 / 2}\right)} \leqslant M C_{0}^{\varepsilon \nu} \varepsilon^{1 / 2} .
\end{aligned}
$$

- If $N=3$ : For all $p \in[2,+\infty[$,

$$
\begin{aligned}
& \left\|\mathcal{P} u^{\varepsilon}-v\right\|_{L^{1}\left(B_{p}^{4 / p+1 / 2}\right)}+\left\|\mathcal{P} u^{\varepsilon}-v\right\|_{L^{\infty}\left(B_{p}^{4 / p-3 / 2}\right)}+\left\|\mathcal{Q} u^{\varepsilon}\right\|_{L^{2}\left(B_{p}^{4 / p-1 / 2}\right)} \\
& \quad+\left\|b^{\varepsilon}\right\|_{L^{2 p /(p-2)}\left(B_{p}^{2 / p-1 / 2}\right)} \leqslant M C_{0}^{\varepsilon \nu} \varepsilon^{1 / 2-1 / p} .
\end{aligned}
$$

- If $N=2$ : For all $p \in[2,6]$,

$$
\begin{aligned}
& \left\|\mathcal{P} u^{\varepsilon}-v\right\|_{L^{1}\left(B_{p}^{5 /(2 p)+3 / 4}\right)}+\left\|\mathcal{P} u^{\varepsilon}-v\right\|_{L^{\infty}\left(B_{p}^{5 /(2 p)-5 / 4}\right)}+\left\|\mathcal{Q} u^{\varepsilon}\right\|_{L^{2}\left(B_{p}^{5 /(2 p)-1 / 4}\right)} \\
& \quad+\left\|b^{\varepsilon}\right\|_{L^{4 p /(p-2)}\left(B_{p}^{3 /(2 p)-3 / 4}\right)} \leqslant M C_{0}^{\varepsilon \nu} \varepsilon^{1 / 4-1 /(2 p)} .
\end{aligned}
$$

Let us give the outlines of the proof. First, an appropriate change of variables enables us to apply Theorem 0.1. Under the smallness assumption (13), we get a global solution $\left(b^{\varepsilon}, u^{\varepsilon}\right)$ in $E_{\varepsilon \nu}^{N / 2}$ uniformly in $\varepsilon$. The existence of a global solution for the limit system (NSI) stems from classical arguments. Since apparently, it has not been written out yet in the framework of $B_{2,1}^{s}$ spaces, we prove it in Section 6.

While, up to this point, the method still works in the periodical setting (and actually provides us with some local weak convergence results, see Remark 3.2), our proof of global strong convergence is specific to $\mathbb{R}^{N}$. Indeed, we shall make use of the dispersive properties of the linear wave equation in the whole space (namely Proposition 2.2).

As expected, the uniform estimates in $E_{\varepsilon \nu}^{N / 2}$ provide uniform bounds in $L^{1}\left(\mathbb{R}^{+} ; B_{2}^{N / 2-1}\right)$ for the right-hand side of the first two equations of (10), and for $\nu \Delta \mathcal{Q} u^{\varepsilon}$. Therefore, according to Proposition 2.2, $\left(b^{\varepsilon}, \mathcal{Q} u^{\varepsilon}\right)$ convergences to zero (in $L^{4}\left(\mathbb{R}^{+} ; B_{\infty}^{-3 / 4}\right)$ if $N=2$ and in a space very close to $L^{2}\left(\mathbb{R}^{+} ; B_{\infty}^{-1 / 2}\right)$ if $\left.N=3\right)$. Next, routine computations based on standard estimates for the heat equation (see Proposition 7.3) allow us to get $\mathcal{P} u^{\varepsilon} \rightarrow v$ in a suitable functional space. Then we can interpolate with the uniform estimates and get a result of convergence for stronger norms.

\subsection{Convergence in the large}

Let us state the complete result we get in any dimension $N \geqslant 2$ :

THEOREM 2.5. - Suppose that $b_{0} \in B_{2}^{N / 2-1} \cap B_{2}^{N / 2+\alpha}, u_{0} \in B_{2}^{N / 2-1} \cap B_{2}^{N / 2-1+\alpha}$ and $f \in L^{1}\left(\mathbb{R}^{+} ; B_{2}^{N / 2-1} \cap B_{2}^{N / 2-1+\alpha}\right)$ for $\left.\left.a \alpha \in\right] 0,1 / 2\right]$ if $\left.N \geqslant 4, \alpha \in\right] 0,1 / 2[$ if $N=3$ and 
$\alpha \in] 0,1 / 6]$ if $N=2$. Suppose that the incompressible system (NSI) with initial datum $\mathcal{P} u_{0}$ and external force $\mathcal{P} f$ has a solution $v \in F_{T_{0}}^{N / 2} \cap F_{T_{0}}^{N / 2+\alpha}$ for a positive $T_{0}$ possibly infinite. Let

$$
\begin{aligned}
& V \stackrel{\text { def }}{=}\|v\|_{F_{T_{0}}^{N / 2} \cap F_{T_{0}}^{N / 2+\alpha}} \text { and } \\
& X^{0} \stackrel{\text { def }}{=}\left\|b_{0}\right\|_{B_{2}^{N / 2-1} \cap B_{2}^{N / 2+\alpha}}+\left\|\mathcal{Q} u_{0}\right\|_{B_{2}^{N / 2-1} \cap B_{2}^{N / 2-1+\alpha}}+\|\mathcal{Q} f\|_{L^{1}\left(B_{2}^{N / 2-1} \cap B_{2}^{N / 2-1+\alpha}\right)} .
\end{aligned}
$$

Then there exists a positive $\varepsilon_{0}$ depending only on $\alpha, \lambda, \mu, P, V$ and $X^{0}$ and such that for all $0<\varepsilon \leqslant \varepsilon_{0}$, system (1) has a unique solution $\left(b^{\varepsilon}, u^{\varepsilon}\right)$ in $E_{\varepsilon \nu, T_{0}}^{N / 2} \cap E_{\varepsilon \nu, T_{0}}^{N / 2+\alpha}$. Moreover, $\mathcal{P} u_{\varepsilon}$ tends to $v$ in $F_{T_{0}}^{N / 2} \cap F_{T_{0}}^{N / 2+\alpha}$ and $\left(b^{\varepsilon}, \mathcal{Q} u_{\varepsilon}\right)$ tends to 0 in $L^{2}\left(0, T_{0} ; B_{\infty}^{\alpha-1 / 2}\right)$ if $N \geqslant 4$, in every $L^{p}\left(0, T_{0} ; B_{\infty}^{\alpha-1+1 / p}\right)$ with $p>2$ if $N=3$ and in $L^{4}\left(0, T_{0} ; B_{\infty}^{\alpha-3 / 4}\right)$ if $N=2$. More precisely, the following bounds hold for a constant $C=C\left(N, \mu, \lambda, \alpha, P, V, X^{0}\right)$ :

$$
\begin{aligned}
& \left\|\left(b^{\varepsilon}, u^{\varepsilon}\right)\right\|_{E_{\varepsilon \nu, T_{0}}^{N / 2} \cap E_{\varepsilon \nu, T_{0}}^{N / 2+\alpha}} \leqslant C, \\
& \left\|\mathcal{P} u^{\varepsilon}-v\right\|_{F_{T_{0}}^{N / 2} \cap F_{T_{0}}^{N / 2+\alpha}} \leqslant C \varepsilon^{2 \alpha /(2+N+2 \alpha)}, \\
& \left\|\left(b^{\varepsilon}, \mathcal{Q} u^{\varepsilon}\right)\right\|_{L_{T}^{2}\left(B_{\infty}^{\alpha-1 / 2}\right)} \leqslant C \varepsilon^{1 / 2} \quad \text { if } N \geqslant 4, \\
& \left\|\left(b^{\varepsilon}, \mathcal{Q} u^{\varepsilon}\right)\right\|_{L_{T}^{p}\left(B_{\infty}^{\alpha-1+1 / p}\right)} \leqslant C \varepsilon^{1 / p} \quad \text { if } N=3 \text { and } p>2, \\
& \left\|\left(b^{\varepsilon}, \mathcal{Q} u^{\varepsilon}\right)\right\|_{L_{T}^{4}\left(B_{\infty}^{\alpha-3 / 4}\right)} \leqslant C \varepsilon^{1 / 4} \quad \text { if } N=2 .
\end{aligned}
$$

In the case $N=2$, one can choose $T_{0}=+\infty$ and the constants $\varepsilon_{0}$ and $C$ depend on $v$ only through the norm of $\mathcal{P} u(0)$ in $B_{2}^{N / 2-1} \cap B_{2}^{N / 2-1+\alpha}$, and of $\mathcal{P} f$ in $L^{1}\left(\mathbb{R}^{+}\right.$; $\left.B_{2}^{N / 2-1} \cap B_{2}^{N / 2-1+\alpha}\right)$.

Sketchy proof. - To avoid the technicalities as much as possible, we shall consider only the case $N=3$ and $\alpha=1 / 2$ and further assume that the endpoint $(r, p, N)=(2, \infty, 3)$ is allowed in Proposition 2.2. We refer to Section 5 for a rigorous and complete proof.

The existence of a solution for $\left(N S C^{\varepsilon}\right)$ on a small time interval (which may depend on $\varepsilon$ ) is ensured by Theorem 0.2 in [7] regardless of the size of the data: the only assumption that we need is that $1+\varepsilon b_{0}$ be bounded away from zero. Since $B_{2}^{N / 2} \hookrightarrow L^{\infty}$, this is certainly true for $\varepsilon$ small enough.

In the four first steps of the proof, we are given two times $T$ and $T_{0}$ (possibly infinite) such that $0<T \leqslant T_{0}$. We shall suppose that $\left(b^{\varepsilon}, u^{\varepsilon}\right)$ is a solution of (1) belonging to $E_{T} \stackrel{\text { def }}{=} E_{\varepsilon \nu, T}^{3 / 2} \cap E_{\varepsilon \nu, T}^{2}$, and that the limit system has a solution $v \in F_{T_{0}} \stackrel{\text { def }}{=} F_{T_{0}}^{3 / 2} \cap F_{T_{0}}^{2}$.

In the first step of the proof, we shall apply the Strichartz estimates (namely Proposition 2.2) to the first two equations of (10). As $B_{\infty}^{0} \hookrightarrow L^{\infty}$, if we assume that the endpoint $(r, p, N)=(2, \infty, 3)$ is allowed, we get a bound in $L^{2}\left(0, T ; L^{\infty}\right)$ for $\varepsilon^{-1 / 2}\left(b^{\varepsilon}, \mathcal{Q} u^{\varepsilon}\right)$ in terms of the norm of $\left(b^{\varepsilon}, u^{\varepsilon}\right)$ in $E_{T}$.

In the second step, we derive a priori bounds for $\varepsilon^{-\beta}\left(\mathcal{P} u^{\varepsilon}-v\right.$ ) (for a suitable $\beta>0$ ) in terms of $\left\|\left(b^{\varepsilon}, u^{\varepsilon}\right)\right\|_{E_{T}}$ and $\|v\|_{F_{T}}$. These bounds may be obtained as a by-product of estimates for the heat equation with first order terms (see Proposition 7.4). Some paradifferential calculus enables us to use the decay in $\varepsilon$ for $\left(b^{\varepsilon}, \mathcal{Q} u^{\varepsilon}\right)$.

The trickiest part (third step) consists in stating a control for $\left(b^{\varepsilon}, u^{\varepsilon}\right)$ in $E_{T}$, in terms of $v$ and initial data. According to step $2, \mathcal{P} u^{\varepsilon} \approx v$ so that the main difficulty lies in the control of $\left(b^{\varepsilon}, \mathcal{Q} u^{\varepsilon}\right)$. Applying inequality (12) fails because a term $\exp \left(C\left\|\nabla u^{\varepsilon}\right\|_{L_{T}^{1}\left(B_{2}^{N / 2}\right)}\right)$ and thus $\exp \left(C\left\|\nabla \mathcal{Q} u^{\varepsilon}\right\|_{L_{T}^{1}\left(B_{2}^{N / 2}\right)}\right)$ appears in the right-hand side of the estimate. There is no way of 
preventing this term to increase too much for small $\varepsilon$. On the other hand, step one gives us a bound for $\varepsilon^{-1 / 2} \mathcal{Q} u^{\varepsilon}$ in $L^{2}\left(0, T ; L^{\infty}\right)$, and, since the solution belongs to $E_{T}$, we also have $|D|^{3 / 2} \mathcal{Q} u^{\varepsilon} \in L^{1}\left(0, T ; L^{\infty}\right)$. An obvious interpolation yields a control in $L^{6 / 5}\left(0, T ; L^{\infty}\right)$ for $\varepsilon^{-1 / 6} \nabla \mathcal{Q} u^{\varepsilon}$. Clearly, we are done if the exponential factor in Proposition 2.1 may be replaced with $\exp \|\nabla u\|_{L_{T}^{6 / 5}\left(L^{\infty}\right)}$. To this end, we replace the convection terms $u \cdot \nabla b$ and $u \cdot \nabla d$ by the paraproducts $T_{u^{j}} \partial_{j} b$ and $T_{u^{j}} \partial_{j} d$ (see the definition in Section 1).

Slight modifications of the proof of Proposition 2.1 show that for the new linear system

$\left(L P H^{\varepsilon}\right)$

$$
\left\{\begin{array}{l}
\partial_{t} b+T_{u^{j}} \partial_{j} b+\frac{\Lambda d}{\varepsilon}=F, \\
\partial_{t} d+T_{u^{j}} \partial_{j} d-\nu \Delta d-\frac{\Lambda b}{\varepsilon}=G,
\end{array}\right.
$$

we have estimates analogous to (12) where the exponential factor has been replaced with $\exp \|\nabla u\|_{L_{T}^{1}\left(L^{\infty}\right)}$. We then use the rough estimate

$$
\left\|\nabla \mathcal{Q} u^{\varepsilon}\right\|_{L_{T}^{1}\left(L^{\infty}\right)} \leqslant T^{1 / 6}\left\|\nabla \mathcal{Q} u^{\varepsilon}\right\|_{L_{T}^{6 / 5}\left(L^{\infty}\right)} \leqslant C(\varepsilon T)^{1 / 6}\left\|\left(b^{\varepsilon}, u^{\varepsilon}\right)\right\|_{E} .
$$

Assuming that we control $\left\|\left(b^{\varepsilon}, u^{\varepsilon}\right)\right\|_{E_{T}}$ and that $T$ is finite, the exponential factor can be made as close of 1 as desired when $\varepsilon$ tends to zero. Actually, a judicious use of Young inequality enables us to replace the $L_{T}^{1}\left(L^{\infty}\right)$ norms for $\nabla u^{\varepsilon}$ with $L_{T}^{r}\left(L^{\infty}\right)$ norms for any finite $r>1$ (plus some other terms which turn out to be nice, see Proposition 4.1 below). Therefore the case $T=+\infty$ may be handled as well.

From this point, the end of the existence and convergence proof is standard. We use a bootstrap argument (fourth step) to close the estimates of the first three steps and a continuity argument (last step) completes the proof.

\section{Global well-posedness and convergence for small data}

This part is devoted to the proof of Theorem 2.4. Throughout the proof, we drop the exponents $\varepsilon$ to simplify the notations.

\section{First step: Existence of a global solution for $\left(N S C^{\varepsilon}\right)$ and uniform estimates}

Let us make the following change of functions:

$$
c(t, x) \stackrel{\text { def }}{=} \varepsilon b\left(\varepsilon^{2} t, \varepsilon x\right), \quad v(t, x) \stackrel{\text { def }}{=} \varepsilon u\left(\varepsilon^{2} t, \varepsilon x\right) \quad \text { and } \quad \bar{f}(t, x) \stackrel{\text { def }}{=} \varepsilon^{3} f\left(\varepsilon^{2} t, \varepsilon x\right) .
$$

Then $(b, u)$ solves (1) if and only if $(c, v)$ solves

$$
\left\{\begin{array}{l}
\partial_{t} c+\operatorname{div} v=-\operatorname{div}(c v), \\
\partial_{t} v+v \cdot \nabla v-\frac{\mu \Delta v+(\lambda+\mu) \nabla \operatorname{div} v}{1+c}+\frac{P^{\prime}(1+c)}{1+c} \nabla c=\bar{f}, \\
\left.(c, v)\right|_{t=0}=\left(c_{0}, v_{0}\right) .
\end{array}\right.
$$

According to Theorem 2.5 of [6], there exist two positive constants $\eta=\eta(N, \lambda, \mu, P)$ and $M=M(N, \lambda, \mu, P)$ such that (14) has a solution $(c, v)$ in $E_{\nu}^{N / 2}$ as soon as

$$
\left\|c_{0}\right\|_{\widetilde{B}_{\nu}^{N / 2, \infty}}+\left\|v_{0}\right\|_{B_{2}^{N / 2-1}}+\|\bar{f}\|_{L^{1}\left(B_{2}^{N / 2-1}\right)} \leqslant \eta .
$$


We moreover have the estimate

$$
\|(c, v)\|_{E_{\nu}^{N / 2}} \leqslant M\left(\left\|c_{0}\right\|_{\widetilde{B}_{\nu}^{N / 2, \infty}}+\left\|v_{0}\right\|_{B_{2}^{N / 2-1}}+\|\bar{f}\|_{L^{1}\left(B_{2}^{N / 2-1}\right)}\right) .
$$

Using (5), (6) and (9), we easily gather that

$$
\left\|c_{0}\right\|_{\widetilde{B}_{\nu}^{N / 2, \infty}}+\left\|v_{0}\right\|_{B_{2}^{N / 2-1}}+\|\bar{f}\|_{L^{1}\left(B_{2}^{N / 2-1}\right)}=\left\|b_{0}\right\|_{\widetilde{B}_{\varepsilon \nu}^{N / 2, \infty}}+\left\|u_{0}\right\|_{B_{2}^{N / 2-1}}+\|f\|_{L^{1}\left(B_{2}^{N / 2-1}\right)}
$$

and

$$
\|(c, v)\|_{E_{\nu}^{N / 2}}=\|(b, u)\|_{E_{\varepsilon \nu}^{N / 2}} .
$$

\section{Second step: Existence of a global solution for (NSI)}

See Proposition 6.1.

\section{Third step: Convergence of $b^{\varepsilon}$ and $\mathcal{Q} u^{\varepsilon}$ to zero}

The main result of this step is given in the following lemma:

LEMMA 3.1. - Let $C_{0}^{\varepsilon \nu}$ be defined as in (13). The solution $(b, u)$ defined in step 1 satisfies

- If $N \geqslant 4$ : For all $p_{N} \leqslant p \leqslant+\infty,\|(b, Q u)\|_{L^{2}\left(B_{p}^{N / p-1 / 2}\right)} \lesssim C_{0}^{\varepsilon \nu} \varepsilon^{1 / 2}$.

- If $N=3$ : For all $2 \leqslant p<+\infty,\|(b, Q u)\|_{L^{2 p /(p-2)}\left(B_{p}^{2 / p-1 / 2}\right)} \lesssim C_{0}^{\varepsilon \nu} \varepsilon^{1 / 2-1 / p}$.

- If $N=2$ : For all $2 \leqslant p \leqslant+\infty,\|(b, Q u)\|_{L^{4 p /(p-2)\left(B_{p}^{3 /(2 p)-3 / 4}\right)}} \lesssim C_{0}^{\varepsilon \nu} \varepsilon^{1 / 4-1 /(2 p)}$.

Proof. - The starting point is the dispersive inequality given in Proposition 2.2 for $\left(W^{\varepsilon}\right)$. Indeed, denoting $d \stackrel{\text { def }}{=} \Lambda^{-1} \operatorname{div} \mathcal{Q} u$, we have

$$
\left\{\begin{array}{l}
\partial_{t} b+\varepsilon^{-1} \Lambda d=F \\
\partial_{t} d-\varepsilon^{-1} \Lambda b=G \\
\left.(b, d)\right|_{t=0}=\left(b_{0}, \Lambda^{-1} \operatorname{div} \mathcal{Q} u_{0}\right)
\end{array}\right.
$$

with $F \stackrel{\text { def }}{=}-\operatorname{div}(b u)$ and $G \stackrel{\text { def }}{=} \nu \Delta d-\Lambda^{-1} \operatorname{div}\left(u \cdot \nabla u+\frac{\varepsilon b}{1+\varepsilon b} \mathcal{A} u+\frac{K(\varepsilon b) \nabla b}{\varepsilon}+f\right)$.

Remark that $\mathcal{Q} u=-\nabla \Lambda^{-1} d$ so that estimating $\mathcal{Q} u$ or $d$ is equivalent (up to an irrelevant constant). On the other hand, if we apply Proposition 2.2 with $s=N / 2-1$ and $r=2$ if $N \geqslant 4$, or $2 \leqslant p<+\infty$ and $r=2 p /(p-2)$ if $N=3$, or $2 \leqslant p \leqslant+\infty$ and $r=4 p /(p-2)$ if $N=2$, we readily obtain the estimates of Lemma 3.1 provided that

$$
\|(F, G)\|_{L^{1}\left(B_{2}^{N / 2-1}\right)} \lesssim C_{0}^{\varepsilon \nu} .
$$

This easily stems from the uniform estimates of step one. Let us just treat the case of the nonlinear terms in $F$ and $G$ which is perhaps not entirely obvious. According to Proposition 1.4, Lemma 1.5 and (7) we have, for small enough $\eta \leqslant 1$,

$$
\begin{gathered}
\|F\|_{L^{1}\left(B_{2}^{N / 2-1}\right)} \lesssim\|b\|_{L^{2}\left(B_{2}^{N / 2}\right)}\|u\|_{L^{2}\left(B_{2}^{N / 2}\right)} \lesssim C_{0}^{\varepsilon \nu}, \\
\|u \cdot \nabla u\|_{L^{1}\left(B_{2}^{N / 2-1}\right)} \lesssim\|u\|_{L^{2}\left(B_{2}^{N / 2}\right)}\|\nabla u\|_{L^{2}\left(B_{2}^{N / 2-1}\right)} \lesssim C_{0}^{\varepsilon \nu},
\end{gathered}
$$




$$
\begin{aligned}
\left\|\frac{\varepsilon b}{1+\varepsilon b} \mathcal{A} u\right\|_{L^{1}\left(B_{2}^{N / 2-1}\right)} & \lesssim \varepsilon\|b\|_{L^{\infty}\left(B_{2}^{N / 2}\right)}\|\mathcal{A} u\|_{L^{1}\left(B_{2}^{N / 2-1}\right)}, \\
& \lesssim \nu^{-1}\|b\|_{L^{\infty}\left(\widetilde{B}_{\varepsilon \nu}^{N / 2, \infty}\right)}\|u\|_{L^{1}\left(B_{2}^{N / 2+1}\right)} \\
& \lesssim C_{0}^{\varepsilon \nu}, \\
\left\|\frac{K(\varepsilon b) \nabla b}{\varepsilon}\right\|_{L^{1}\left(B_{2}^{N / 2-1}\right)} & \lesssim\|b\|_{L^{2}\left(B_{2}^{N / 2}\right)}\|\nabla b\|_{L^{2}\left(B_{2}^{N / 2-1}\right)} \lesssim C_{0}^{\varepsilon \nu} .
\end{aligned}
$$

\section{Last step: Convergence of the incompressible part}

Let $w \stackrel{\text { def }}{=} \mathcal{P} u-v$. Applying Leray projector to the second equation of (1) and subtracting (NSI) from it yields the following equation for $w$ :

$$
\left\{\begin{array}{l}
\partial_{t} w-\mu \Delta w=H \\
\left.w\right|_{t=0}=0
\end{array}\right.
$$

with $H \stackrel{\text { def }}{=}-\mathcal{P}(w \cdot \nabla v)-\mathcal{P}(u \cdot \nabla w)-\mathcal{P}(\mathcal{Q} u \cdot \nabla v)-\mathcal{P}(u \cdot \nabla \mathcal{Q} u)-\mathcal{P}\left(\frac{\varepsilon b}{1+\varepsilon b} \mathcal{A} u\right)$.

Let us first treat the case $N \geqslant 4$ which is the easiest to handle. We are going to prove that, for $p_{N} \leqslant p \leqslant+\infty$,

$$
Y_{p} \stackrel{\text { def }}{=}\|w\|_{L^{1}\left(B_{p}^{N / p+1 / 2}\right)}+\|w\|_{L^{\infty}\left(B_{p}^{N / p-3 / 2}\right)} \lesssim C_{0}^{\varepsilon \nu} \varepsilon^{1 / 2} .
$$

According to Proposition 7.3, we have

$$
Y_{p} \lesssim\|H\|_{L^{1}\left(B_{p}^{N / p-3 / 2}\right)}
$$

Thanks to Proposition 1.4 and to the estimates of steps 1, 2 and 3, we gather

$$
\begin{aligned}
\|\mathcal{P}(w \cdot \nabla v)\|_{L^{1}\left(B_{p}^{N / p-3 / 2}\right)} & \lesssim\|\nabla v\|_{L^{2}\left(B_{2}^{N / 2-1}\right)}\|w\|_{L^{2}\left(B_{p}^{N / p-1 / 2}\right)} \\
\|\mathcal{P}(u \cdot \nabla w)\|_{L^{1}\left(B_{p}^{N / p-3 / 2}\right)} & \lesssim\|u\|_{L^{2}\left(B_{2}^{N / 2}\right)}\|\nabla w\|_{L^{2}\left(B_{p}^{N / p-3 / 2}\right)} \\
\|\mathcal{P}(\mathcal{Q} u \cdot \nabla v)\|_{L^{1}\left(B_{p}^{N / p-3 / 2}\right)} & \lesssim\|\nabla v\|_{L^{2}\left(B_{2}^{N / 2-1}\right)}\|\mathcal{Q} u\|_{L^{2}\left(B_{p}^{N / p-1 / 2}\right)} \\
& \lesssim\left(C_{0}^{\varepsilon \nu}\right)^{2} \varepsilon^{1 / 2} \\
\|\mathcal{P}(u \cdot \nabla \mathcal{Q} u)\|_{L^{1}\left(B_{p}^{N / p-3 / 2}\right)} & \lesssim\|u\|_{L^{2}\left(B_{2}^{N / 2}\right)}\|\nabla \mathcal{Q} u\|_{L^{2}\left(B_{2}^{N / p-3 / 2}\right)} \\
& \lesssim\left(C_{0}^{\varepsilon \nu}\right)^{2} \varepsilon^{1 / 2}
\end{aligned}
$$

Note that all the above estimates are justified since $N / 2+N / p-3 / 2>0$ for any $p \leqslant+\infty$ when $N \geqslant 4$. Thanks to the embedding $B_{2}^{N / 2-3 / 2} \hookrightarrow B_{p}^{N / p-3 / 2}$ and to (7), we also have

$$
\begin{aligned}
\left\|\mathcal{P}\left(\frac{\varepsilon b}{1+\varepsilon b} \mathcal{A} u\right)\right\|_{L^{1}\left(B_{p}^{N / p-3 / 2}\right)} & \lesssim\left\|\frac{\varepsilon b}{1+\varepsilon b} \mathcal{A} u\right\|_{L^{1}\left(B_{2}^{N / 2-3 / 2}\right)} \\
& \lesssim\|\varepsilon b\|_{L^{4}\left(B_{2}^{N / 2}\right)}\|\mathcal{A} u\|_{L^{4 / 3}\left(B_{2}^{N / 2-3 / 2}\right)} \\
& \lesssim \varepsilon^{1 / 2}\|b\|_{L^{4}\left(\widetilde{B}_{\varepsilon \nu}^{N / 2,4}\right)}\|u\|_{L^{4 / 3}\left(B_{2}^{N / 2+1 / 2}\right)} \\
& \lesssim\left(C_{0}^{\varepsilon \nu}\right)^{2} \varepsilon^{1 / 2}
\end{aligned}
$$


Plugging all the above estimates in (18), we gather

$$
\begin{aligned}
Y_{p} & \leqslant C\left(\left(C_{0}^{\varepsilon \nu}\right)^{2} \varepsilon^{1 / 2}+\left(\|u\|_{L^{2}\left(B_{2}^{N / 2}\right)}+\|v\|_{L^{2}\left(B_{2}^{N / 2}\right)}\right) Y_{p}\right), \\
& \leqslant C C_{0}^{\varepsilon \nu} \varepsilon^{1 / 2}+C \eta Y_{p},
\end{aligned}
$$

so that we can conclude to (17) provided that the constant $\eta$ has been chosen small enough. Let us remark that, in particular, $\mathcal{P} u$ tends to $v$ in $L^{1}\left(\mathbb{R}^{+} ; B_{\infty}^{1 / 2}\right) \cap C\left([0,+\infty] ; B_{\infty}^{-3 / 2}\right)$. Interpolating with the estimates of step one (and using some embeddings), we see that convergence actually holds in $L^{1}\left(\mathbb{R}^{+} ; B_{\infty}^{1-\alpha}\right) \cap C\left([0,+\infty] ; B_{\infty}^{-1-\alpha}\right)$ for all $\left.\left.\alpha \in\right] 0,1 / 2\right]$.

In fact, one can even prove that $\mathcal{P} u$ tends to 0 in $C\left([0,+\infty] ; B_{\infty}^{-1}\right)$. Indeed, according to (10) and using arguments similar to those of step two, one can prove that $\mathcal{P} u$ solves a heat equation with right-hand side uniformly bounded in $L^{1}\left(B_{2}^{N / 2-1}\right.$ ) (thus in $L^{1}\left(B_{\infty}^{-1}\right)$ ) for small $\varepsilon$. According to Proposition 7.3, this implies that

$$
\sum_{q \in \mathbb{Z}} 2^{-q}\left\|\Delta_{q} \mathcal{P} u\right\|_{L^{\infty}\left(\mathbb{R}^{+} \times \mathbb{R}^{N}\right)} \leqslant C<+\infty \quad \text { uniformly in } \varepsilon .
$$

Since each $\Delta_{q} \mathcal{P} u$ tends to $\Delta_{q} v$ in $C\left([0,+\infty] ; B_{\infty}^{-3 / 2}\right)$, thus also in $C\left([0,+\infty] ; B_{\infty}^{-1}\right)$, we can conclude by Lebesgue theorem, that $\mathcal{P} u$ tends to $v$ in $C\left([0,+\infty] ; B_{\infty}^{-1}\right)$.

In the case $N=2,3$, Proposition 2.2 does not provide us with estimates for $\varepsilon^{-1 / 2} \mathcal{Q} u$ in $L^{2}\left(\mathbb{R}^{+} ; B_{p}^{N / p-1 / 2}\right)$. On the other hand, one can interpolate the following estimate for $u$

$$
\|u\|_{L^{1}\left(B_{2}^{N / 2+1}\right)} \lesssim C_{0}^{\varepsilon \nu}
$$

given by step one, with the dispersive inequalities of Lemma 3.1. This still gives us some decay in $\varepsilon$.

Let us first treat the case $N=3$. Use the following interpolation for $2 \leqslant q \leqslant+\infty$

$$
L^{2}\left(\mathbb{R}^{+} ; B_{(q+2) / 2}^{(14-q) /(2 q+4)}\right)=\left[L^{1}\left(\mathbb{R}^{+} ; B_{2}^{5 / 2}\right) ; L^{2 q /(q-2)}\left(\mathbb{R}^{+} ; B_{q}^{-1 / 2+2 / q}\right)\right]_{2 /(q+2)} .
$$

Make the change of parameter $p=(q+2) / 2$. Thanks to Lemma 3.1 and estimate (19), we conclude that

$$
\|\mathcal{Q} u\|_{L^{2}\left(B_{p}^{4 / p-1 / 2}\right)} \lesssim C_{0}^{\varepsilon \nu} \varepsilon^{1 / 2-1 / p} \quad \text { for all } 2 \leqslant p<+\infty .
$$

Let us prove that $w$ tends to zero in the space $L^{1}\left(\mathbb{R}^{+} ; B_{p}^{4 / p+1 / 2}\right) \cap C\left(\mathbb{R}^{+} ; B_{p}^{4 / p-3 / 2}\right)$ $(2 \leqslant p<+\infty)$. More precisely, we want to prove that

$$
Y_{p} \stackrel{\text { def }}{=}\|w\|_{L^{1}\left(B_{p}^{4 / p+1 / 2}\right)}+\|w\|_{L^{\infty}\left(B_{p}^{4 / p-3 / 2}\right)} \lesssim C_{0}^{\varepsilon \nu} \varepsilon^{1 / 2-1 / p} .
$$

According to Proposition 1.4 and to the estimates of steps 1, 2 and 3, we have

$$
\begin{aligned}
\|\mathcal{P}(w \cdot \nabla v)\|_{L^{1}\left(B_{p}^{4 / p-3 / 2}\right)} & \lesssim\|\nabla v\|_{L^{2}\left(B_{2}^{1 / 2}\right)}\|w\|_{L^{2}\left(B_{p}^{4 / p-1 / 2}\right)} \\
\|\mathcal{P}(u \cdot \nabla w)\|_{L^{1}\left(B_{p}^{4 / p-3 / 2}\right)} & \lesssim\|u\|_{L^{2}\left(B_{2}^{3 / 2}\right)}\|\nabla w\|_{L^{2}\left(B_{p}^{4 / p-3 / 2}\right)} \\
\|\mathcal{P}(\mathcal{Q} u \cdot \nabla v)\|_{L^{1}\left(B_{p}^{4 / p-3 / 2}\right)} & \lesssim\|\nabla v\|_{L^{2}\left(B_{2}^{1 / 2}\right)}\|\mathcal{Q} u\|_{L^{2}\left(B_{p}^{4 / p-1 / 2}\right)} \\
& \lesssim\left(C_{0}^{\varepsilon \nu}\right)^{2} \varepsilon^{1 / 2-1 / p}
\end{aligned}
$$




$$
\begin{aligned}
\|\mathcal{P}(u \cdot \nabla \mathcal{Q} u)\|_{L^{1}\left(B_{p}^{4 / p-3 / 2}\right)} & \lesssim\|u\|_{L^{2}\left(B_{2}^{3 / 2}\right)}\|\nabla \mathcal{Q} u\|_{L^{2}\left(B_{2}^{4 / p-3 / 2}\right)}, \\
& \lesssim\left(C_{0}^{\varepsilon \nu}\right)^{2} \varepsilon^{1 / 2-1 / p}
\end{aligned}
$$

Thanks to the embedding $B_{2}^{1 / p} \hookrightarrow B_{p}^{4 / p-3 / 2}$, we also have

$$
\begin{aligned}
\left\|\mathcal{P}\left(\frac{\varepsilon b}{1+\varepsilon b} \mathcal{A} u\right)\right\|_{L^{1}\left(B_{p}^{4 / p-3 / 2}\right)} & \lesssim\|\varepsilon b\|_{L^{4 p /(p-2)}\left(B_{2}^{3 / 2}\right)}\|\mathcal{A} u\|_{L^{4 p /(3 p+2)}\left(B_{2}^{1 / p}\right)}, \\
& \lesssim \varepsilon^{1 / 2-1 / p}\|b\|_{L^{4 p /(p-2)}\left(\widetilde{B}_{\varepsilon \nu}^{3 / 2,4 p /(p-2)}\right)}\|u\|_{L^{4 p /(3 p+2)}\left(B_{2}^{1 / p+2}\right)}, \\
& \lesssim\left(C_{0}^{\varepsilon \nu}\right)^{2} \varepsilon^{1 / 2-1 / p} .
\end{aligned}
$$

Applying Proposition 7.3 to (16) and using the above estimates, we gather

$$
Y_{p} \leqslant C C_{0}^{\varepsilon \nu} \varepsilon^{1 / 2-1 / p}+C \eta Y_{p},
$$

so that we can conclude to (17) provided that the constant $\eta$ has been chosen small enough.

By embedding, we readily have $\mathcal{P} u \rightarrow v$ in $L^{1}\left(\mathbb{R}^{+} ; B_{\infty}^{1-\alpha}\right) \cap C\left([0,+\infty] ; B_{\infty}^{-1-\alpha}\right)$ for all $\alpha \in] 0,1 / 2[$. The borderline case $\alpha=0$ may be reached as in the case $N \geqslant 4$.

Let us complete the proof with the study of case $N=2$. Use the following interpolation for $2 \leqslant q \leqslant+\infty$

$$
L^{2}\left(\mathbb{R}^{+} ; B_{(6 q+4) /(q+6)}^{(14+q) /(6 q+4)}\right)=\left[L^{1}\left(\mathbb{R}^{+} ; B_{2}^{2}\right) ; L^{4 q /(q-2)}\left(\mathbb{R}^{+} ; B_{q}^{-3 / 4+3 /(2 q)}\right)\right]_{(q+2) /(3 q+2)} .
$$

Make the change of parameter $p=(6 q+4) /(q+6)$. Thanks to Lemma 3.1 and estimate (19), we get

$$
\|\mathcal{Q} u\|_{L^{2}\left(B_{p}^{5 /(2 p)-1 / 4}\right)} \lesssim C_{0}^{\varepsilon \nu} \varepsilon^{1 / 4-1 /(2 p)} \quad \text { for all } 2 \leqslant p \leqslant 6 .
$$

Next, we are going to prove that

$$
Y_{p} \stackrel{\text { def }}{=}\|w\|_{L^{1}\left(B_{p}^{5 /(2 p)+3 / 4}\right)}+\|w\|_{L^{\infty}\left(B_{p}^{5 /(2 p)-5 / 4}\right)} \lesssim C_{0}^{\varepsilon \nu} \varepsilon^{1 / 4-1 /(2 p)} .
$$

According to Proposition 1.4 and to the estimates of steps 1, 2 and 3, we have

$$
\begin{aligned}
\|\mathcal{P}(w \cdot \nabla v)\|_{L^{1}\left(B_{p}^{5 /(2 p)-5 / 4}\right)} & \lesssim\|\nabla v\|_{L^{2}\left(B_{2}^{0}\right)}\|w\|_{L^{2}\left(B_{p}^{5 /(2 p)-1 / 4}\right)} \\
\|\mathcal{P}(u \cdot \nabla w)\|_{L^{1}\left(B_{p}^{5 /(2 p)-5 / 4}\right)} & \lesssim\|u\|_{L^{2}\left(B_{2}^{1}\right)}\|\nabla w\|_{L^{2}\left(B_{p}^{5 /(2 p)-5 / 4}\right)} \\
\|\mathcal{P}(\mathcal{Q} u \cdot \nabla v)\|_{L^{1}\left(B_{p}^{5 /(2 p)-5 / 4}\right)} & \lesssim\|\nabla v\|_{L^{2}\left(B_{2}^{0}\right)}\|\mathcal{Q} u\|_{L^{2}\left(B_{p}^{5 /(2 p)-1 / 4}\right)} \\
& \lesssim\left(C_{0}^{\varepsilon \nu}\right)^{2} \varepsilon^{1 / 4-1 /(2 p)} \\
\|\mathcal{P}(u \cdot \nabla \mathcal{Q} u)\|_{L^{1}\left(B_{p}^{5 /(2 p)-5 / 4}\right)} & \lesssim\|u\|_{L^{2}\left(B_{2}^{1}\right)}\|\nabla \mathcal{Q} u\|_{L^{2}\left(B_{2}^{5 /(2 p)-5 / 4}\right)} \\
& \lesssim\left(C_{0}^{\varepsilon \nu}\right)^{2} \varepsilon^{1 / 4-1 /(2 p)}
\end{aligned}
$$

Note that the condition $5 / 2 p-1 / 4>0$ is always satisfied for $p \leqslant 6$. Thanks to the embedding $B_{2}^{1 /(2 p)-1 / 4} \hookrightarrow B_{p}^{5 /(2 p)-5 / 4}$, we also have 


$$
\begin{aligned}
& \left\|\mathcal{P}\left(\frac{\varepsilon b}{1+\varepsilon b} \mathcal{A} u\right)\right\|_{L^{1}\left(B_{p}^{5 /(2 p)-5 / 4}\right)} \\
& \quad \lesssim\|\varepsilon b\|_{L^{8 p /(p-2)}\left(B_{2}^{1}\right)}\|\mathcal{A} u\|_{L^{8 p /(7 p+2)}\left(B_{2}^{1 /(2 p)-1 / 4}\right)}, \\
& \quad \lesssim \varepsilon^{1 / 4-1 /(2 p)}\|b\|_{L^{8 p /(p-2)}\left(\widetilde{B}_{\varepsilon \nu}^{1,8 p /(p-2)}\right)}\|u\|_{L^{8 p /(7 p+2)}\left(B_{2}^{1 /(2 p)+7 / 4}\right)}, \\
& \quad \lesssim\left(C_{0}^{\varepsilon \nu}\right)^{2} \varepsilon^{1 / 4-1 /(2 p)} .
\end{aligned}
$$

Applying Proposition 7.3 and using all the above estimates, we can conclude to the desired inequality exactly as in the case $N \geqslant 3$.

By embedding, we get $\mathcal{P} u \rightarrow v$ in $L^{1}\left(\mathbb{R}^{+} ; B_{\infty}^{1-\alpha}\right) \cap C\left([0,+\infty] ; B_{\infty}^{-1-\alpha}\right)$ for all $\left.\alpha \in\right] 0,1 / 6[$. The borderline case $\alpha=0$ may be reached by repeating the arguments of case $N \geqslant 4$.

Remark 3.2. - The first step also holds in the periodic setting. That means that in both cases (periodic or whole space), uniform estimates are available for $\left(b^{\varepsilon}, u^{\varepsilon}\right)$ in, say,

$$
\left(C_{b}\left(\mathbb{R}^{+} ; B^{N / 2-1}\right) \cap L^{2}\left(\mathbb{R}^{+} ; B^{N / 2}\right)\right) \times\left(C_{b}\left(\mathbb{R}^{+} ; B^{N / 2-1}\right) \cap L^{1}\left(\mathbb{R}^{+} ; B^{N / 2+1}\right)\right)^{N} .
$$

Following the arguments of P.-L. Lions and N. Masmoudi in [28], we can get some results of convergence (local in time and space) to the incompressible solution. Since in our framework the incompressible solution is unique, the whole sequence $\left(\rho^{\varepsilon}, u^{\varepsilon}\right)$ converges to $(1, v)$. On the other hand, as the method introduced in [28] requires some a priori bounds on the sequence $\left(\rho^{\varepsilon}, u^{\varepsilon}\right)$, it is unlikely to help us to treat the case of large initial data.

\section{Estimates for the linearized system}

The main result of this section is the following proposition which is at the root of the proof of Theorem 2.5:

Proposition 4.1. - Let $\varepsilon>0, s \in \mathbb{R}, 1 \leqslant p, r<+\infty$ and $(c, d)$ be a solution of

$\left(L P H^{\varepsilon}\right)$

$$
\left\{\begin{array}{l}
\partial_{t} c+T_{u^{j}} \partial_{j} c+\frac{\Lambda d}{\varepsilon}=F \\
\partial_{t} d+T_{u^{j}} \partial_{j} d-\nu \Delta d-\frac{\Lambda c}{\varepsilon}=G .
\end{array}\right.
$$

Then there exists a constant $C$ depending only on $N, p, r$ and $s$, and such that the following estimate holds:

$$
\begin{aligned}
& \|c(t)\|_{\widetilde{B}_{\varepsilon, \nu}^{s, \infty}}+\|d(t)\|_{B_{2}^{s-1}}+\nu \int_{0}^{t}\left(\|c(\tau)\|_{\widetilde{B}_{\varepsilon, \nu}^{s, 1}}+\|d(\tau)\|_{B_{2}^{s+1}}\right) \mathrm{d} \tau \\
& \leqslant C \mathrm{e}^{C V_{\nu, \varepsilon}^{p, r}(t)}\left(\left\|c_{0}\right\|_{\widetilde{B}_{\varepsilon \nu}^{s, \infty}}+\left\|d_{0}\right\|_{B_{2}^{s-1}}+\int_{0}^{t} \mathrm{e}^{-C V_{\nu, \varepsilon}^{p, r}(\tau)}\left(\|F(\tau)\|_{\widetilde{B}_{\varepsilon \nu}^{s, \infty}}+\|G(\tau)\|_{B_{2}^{s-1}}\right) \mathrm{d} \tau\right),
\end{aligned}
$$

where, if $p>1$,

$$
V_{\nu, \varepsilon}^{p, r}(t) \stackrel{\text { def }}{=} \int_{0}^{t}\left(\nu^{1-p}\|\nabla u(\tau)\|_{B_{\infty, \infty}^{2 / p-2}}^{p}+\left(\varepsilon^{2} \nu\right)^{r-1}\|\nabla u(\tau)\|_{L^{\infty}}^{r}\right) \mathrm{d} \tau
$$


and

$$
V_{\nu, \varepsilon}^{1, r}(t) \stackrel{\text { def }}{=} \int_{0}^{t}\left(\|\nabla u(\tau)\|_{L^{\infty}}+\left(\varepsilon^{2} \nu\right)^{r-1}\|\nabla u(\tau)\|_{L^{\infty}}^{r}\right) \mathrm{d} \tau .
$$

Proof. - It suffices to consider the case $\varepsilon=1$. Indeed, one can make the change of functions

$$
\begin{gathered}
\tilde{c}(t, x) \stackrel{\text { def }}{=} \varepsilon c\left(\varepsilon^{2} t, \varepsilon x\right), \quad \tilde{d}(t, x) \stackrel{\text { def }}{=} \varepsilon d\left(\varepsilon^{2} t, \varepsilon x\right), \quad \tilde{u}(t, x) \stackrel{\text { def }}{=} \varepsilon u\left(\varepsilon^{2} t, \varepsilon x\right), \\
\widetilde{F}(t, x) \stackrel{\text { def }}{=} \varepsilon^{3} F\left(\varepsilon^{2} t, \varepsilon x\right), \quad \widetilde{G}(t, x) \stackrel{\text { def }}{=} \varepsilon^{3} G\left(\varepsilon^{2} t, \varepsilon x\right) .
\end{gathered}
$$

Then $(\tilde{c}, \tilde{d})$ solves

$$
\left(L P H^{1}\right) \quad\left\{\begin{array}{l}
\partial_{t} \tilde{c}+T_{\tilde{u}^{j}} \partial_{j} \tilde{c}+\Lambda \tilde{d}=\widetilde{F}, \\
\partial_{t} \tilde{d}+T_{\tilde{u}^{j}} \partial_{j} \tilde{d}-\nu \Delta \tilde{d}-\Lambda \tilde{c}=\widetilde{G} .
\end{array}\right.
$$

Now, if Proposition 4.1 has been shown to hold in the case $\varepsilon=1$ then scaling arguments (see (5), (6) and (9)) enable us to conclude.

Let us tackle the proof in the case $\varepsilon=1$. It is actually quite similar to the one of Proposition 2.3 in [6] (where we considered only the case $\varepsilon=r=p=1$ and kept the full convection terms $u \cdot \nabla z$ instead of $T_{u^{j}} \partial_{j} z$ here).

To avoid a tedious distinction between the case $p>1$ and the case $p=1$, it will be meant throughout the proof that $\|\nabla u\|_{B_{\infty, \infty}^{2 / p-2}}$ stands for $\|\nabla u\|_{L^{\infty}}$ if $p=1$.

Let $q_{0} \stackrel{\text { def }}{=} 1-\left[\log _{2} \nu\right]$. Clearly, we need appropriate estimates for

$$
\begin{aligned}
& k_{q}=\sqrt{\left\|\Delta_{q} c\right\|_{L^{2}}^{2}+\left\|\Delta_{q} d\right\|_{L^{2}}^{2}} \quad \text { if } q \leqslant q_{0}-1, \\
& k_{q}=\sqrt{\left\|\nu \Lambda \Delta_{q} c\right\|_{L^{2}}^{2}+\left\|\Delta_{q} d\right\|_{L^{2}}^{2}} \quad \text { if } q \geqslant q_{0} .
\end{aligned}
$$

This suggests us to write evolution equations for $\Delta_{q} c$ and $\Delta_{q} d$. Applying the operator $\Delta_{q}$ to $(\mathrm{LPH})$, we infer that $\left(\Delta_{q} c, \Delta_{q} d\right)$ satisfies

$$
\left\{\begin{array}{l}
\partial_{t} \Delta_{q} c+\Delta_{q}\left(T_{u^{j}} \partial_{j} c\right)+\Lambda \Delta_{q} d=\Delta_{q} F \\
\partial_{t} \Delta_{q} d+\Delta_{q}\left(T_{u^{j}} \partial_{j} d\right)-\nu \Delta_{q} d-\Lambda \Delta_{q} c=\Delta_{q} G .
\end{array}\right.
$$

It turns out that rough energy arguments applied to $\left(L P H_{q}\right)$ do not yield appropriate estimates for $k_{q}$. The reason why is that the linear operator associated to (LPH) is not diagonal. To overcome this difficulty, we shall follow [6] and use

$$
\begin{aligned}
& f_{q}=\sqrt{\left\|\Delta_{q} c\right\|_{L^{2}}^{2}+\left\|\Delta_{q} d\right\|_{L^{2}}^{2}-\frac{1}{4}\left(\nu \Lambda \Delta_{q} c \mid \Delta_{q} d\right)} \text { for } q \leqslant q_{0}-1, \\
& f_{q}=\sqrt{\left\|\nu \Lambda \Delta_{q} c\right\|_{L^{2}}^{2}+2\left\|\Delta_{q} d\right\|_{L^{2}}^{2}-2\left(\nu \Lambda \Delta_{q} c \mid \Delta_{q} d\right)} \quad \text { for } q \geqslant q_{0},
\end{aligned}
$$

where $(a \mid b)$ stands for the scalar product in $L^{2}\left(\mathbb{R}^{N}\right)$.

Using Supp $\mathcal{F}\left(\Delta_{q} c\right) \subset 2^{q} C(0,5 / 6,12 / 5)$ and the definition of $q_{0}$, we gather

$$
C^{-1} k_{q} \leqslant f_{q} \leqslant C k_{q}
$$

for a universal constant $C$. 
The first two steps of the proof are devoted to getting a universal positive constant $\kappa$ such that for any positive $K$, the following inequality holds true:

$$
\begin{aligned}
& \frac{1}{2} \frac{\mathrm{d}}{\mathrm{d} t} f_{q}^{2}+\kappa \nu \min \left(2^{2 q}, \nu^{-2}\right) k_{q} f_{q} \\
& \lesssim f_{q}\left(\max \left(1,2^{q} \nu\right)\left\|\Delta_{q} F\right\|_{L^{2}}+\left\|\Delta_{q} G\right\|_{L^{2}}+\frac{\kappa \nu}{K} \min \left(2^{2 q}, \nu^{-2}\right) \sum_{\left|q^{\prime}-q\right| \leqslant 3} k_{q^{\prime}}\right. \\
& \left.\quad+\left[\left(\frac{K}{\kappa \nu}\right)^{p-1}\|\nabla u\|_{B_{\infty, \infty}^{2 / p-2}}^{p}+\left(\frac{\nu K}{\kappa}\right)^{r-1}\|\nabla u\|_{L^{\infty}}^{r}\right] \sum_{\left|q^{\prime}-q\right| \leqslant 3} k_{q^{\prime}}\right) .
\end{aligned}
$$

We will then show (third step) that this inequality entails a decay for $c$ and a low-frequencies damping for $d$.

In the last part of the proof (fourth step), we will use step three to show that (LPH) has indeed a smoothing parabolic effect on $d$ with the gain of two derivatives.

\section{First step: low frequencies}

We here suppose that $q<q_{0}$ (thus $2^{q} \nu<2$ ). According to [6], we have the following energy equality:

$$
\begin{aligned}
\frac{1}{2} \frac{\mathrm{d}}{\mathrm{d} t} & f_{q}^{2}+\frac{7 \nu}{8}\left\|\Lambda \Delta_{q} d\right\|_{L^{2}}^{2}+\frac{\nu}{8}\left\|\Lambda \Delta_{q} c\right\|_{L^{2}}^{2}-\frac{\nu^{2}}{8}\left(\Lambda^{2} \Delta_{q} d \mid \Lambda \Delta_{q} c\right) \\
= & \left(\Delta_{q} F \mid \Delta_{q} c\right)+\left(\Delta_{q} G \mid \Delta_{q} d\right)-\frac{1}{8}\left(\left(\nu \Lambda \Delta_{q} d \mid \Delta_{q} F\right)+\left(\nu \Lambda \Delta_{q} c \mid \Delta_{q} G\right)\right) \\
& -\left(\Delta_{q}\left(T_{u^{j}} \partial_{j} c\right) \mid \Delta_{q} c\right)-\left(\Delta_{q}\left(T_{u^{j}} \partial_{j} d\right) \mid \Delta_{q} d\right) \\
& +\frac{1}{8}\left(\left(\nu \Lambda \Delta_{q} c \mid \Delta_{q}\left(T_{u^{j}} \partial_{j} d\right)\right)+\left(\nu \Lambda \Delta_{q}\left(T_{u^{j}} \partial_{j} c\right) \mid \Delta_{q} d\right)\right)
\end{aligned}
$$

Using the fact that $2^{q} \nu<2$, we have

$$
\left\|\nu \Lambda \Delta_{q} c\right\|_{L^{2}} \lesssim\left\|\Delta_{q} c\right\|_{L^{2}} \quad \text { and } \quad\left\|\nu \Lambda \Delta_{q} d\right\|_{L^{2}} \lesssim\left\|\Delta_{q} d\right\|_{L^{2}}
$$

Now, (23) enables us to bound the first four terms of the right-hand side of (25) by

$$
C f_{q}\left(\left\|\Delta_{q} F\right\|_{L^{2}}+\left\|\Delta_{q} G\right\|_{L^{2}}\right) .
$$

Let us remark that for a suitable constant $\kappa$, we have

$$
\frac{7 \nu}{8}\left\|\Lambda \Delta_{q} d\right\|_{L^{2}}^{2}+\frac{\nu}{8}\left\|\Lambda \Delta_{q} c\right\|_{L^{2}}^{2}-\frac{\nu^{2}}{8}\left(\Lambda^{2} \Delta_{q} d \mid \Lambda \Delta_{q} c\right) \geqslant \kappa \nu k_{q} f_{q} .
$$

To bound the remaining terms of the right-hand side (i.e. the convection terms), we use Lemma 7.5. After an obvious integration by parts, we end up with

$$
\begin{aligned}
\left|\left(\Delta_{q}\left(T_{u^{j}} \partial_{j} c\right) \mid \Delta_{q} c\right)\right| \lesssim & \left\|S_{q-1} \operatorname{div} u\right\|_{L^{\infty}}\left\|\Delta_{q} c\right\|_{L^{2}}^{2} \\
& +2^{2 q(1-1 / p)}\|\nabla u\|_{B_{\infty, \infty}^{2 / p-2}}\left\|\Delta_{q} c\right\|_{L^{2}} \sum_{\left|q^{\prime}-q\right| \leqslant 3}\left\|\Delta_{q^{\prime}} c\right\|_{L^{2}}, \\
\left|\left(\Delta_{q}\left(T_{u^{j}} \partial_{j} d\right) \mid \Delta_{q} d\right)\right| \lesssim & \left\|S_{q-1} \operatorname{div} u\right\|_{L^{\infty}}\left\|\Delta_{q} d\right\|_{L^{2}}^{2} \\
& +2^{2 q(1-1 / p)}\|\nabla u\|_{B_{\infty, \infty}^{2 / p-2}}\left\|\Delta_{q} d\right\|_{L^{2}} \sum_{\left|q^{\prime}-q\right| \leqslant 3}\left\|\Delta_{q^{\prime}} d\right\|_{L^{2}},
\end{aligned}
$$




$$
\begin{aligned}
& \left|\left(\nu \Lambda \Delta_{q} c \mid \Delta_{q}\left(T_{u^{j}} \partial_{j} d\right)\right)+\left(\Lambda \Delta_{q}\left(T_{u^{j}} \partial_{j} c\right) \mid \Delta_{q} d\right)\right| \\
& \lesssim\left\|S_{q-1} \operatorname{div} u\right\|_{L^{\infty}}\left\|\nu \Lambda \Delta_{q} c\right\|_{L^{2}}\left\|\Delta_{q} d\right\|_{L^{2}} \\
& \quad+2^{2 q(1-1 / p)}\|\nabla u\|_{B_{\infty, \infty}^{2 / p-2}}\left(\left\|\nu \Lambda \Delta_{q} c\right\|_{L^{2}} \sum_{\left|q^{\prime}-q\right| \leqslant 3}\left\|\Delta_{q^{\prime}} d\right\|_{L^{2}}\right. \\
& \left.\quad+\left\|\Delta_{q} d\right\|_{L^{2}} \sum_{\left|q^{\prime}-q\right| \leqslant 3}\left\|\nu \Lambda \Delta_{q^{\prime}} c\right\|_{L^{2}}\right) .
\end{aligned}
$$

Using again (26), some further computations enable us to bound the convection terms by

$$
C 2^{2 q(1-1 / p)}\|\nabla u\|_{B_{\infty, \infty}^{2 / p-2}} f_{q} \sum_{\left|q^{\prime}-q\right| \leqslant 3} k_{q^{\prime}} .
$$

Plugging all these inequalities in (25) yields

$$
\begin{aligned}
& \frac{1}{2} \frac{\mathrm{d}}{\mathrm{d} t} f_{q}^{2}+\kappa \nu 2^{2 q} k_{q} f_{q} \\
& \quad \lesssim f_{q}\left(\left\|\Delta_{q} F\right\|_{L^{2}}+\left\|\Delta_{q} G\right\|_{L^{2}}+2^{2 q(1-1 / p)}\|\nabla u\|_{B_{\infty, \infty}^{2 / p-2}} \sum_{\left|q^{\prime}-q\right| \leqslant 3} k_{q^{\prime}}\right) .
\end{aligned}
$$

Moreover, according to Young inequality, we have for all $K>0$,

$$
2^{2 q(1-1 / p)}\|\nabla u\|_{B_{\infty, \infty}^{2 / p-2}} \leqslant \frac{1}{p}\left(\frac{K}{\kappa \nu}\right)^{p-1}\|\nabla u\|_{B_{\infty, \infty}^{2 / p-2}}^{p}+\frac{(p-1) \kappa \nu}{p K} 2^{2 q} .
$$

This completes the proof of (24) in the case $q<q_{0}$.

\section{Second step: high frequencies}

Suppose that $q \geqslant q_{0}$ (hence $2^{q} \nu \geqslant 2$ ). Following [6], we obtain

$$
\begin{aligned}
\frac{1}{2} \frac{\mathrm{d}}{\mathrm{d} t} & f_{q}^{2}+\nu\left\|\Lambda \Delta_{q} c\right\|_{L^{2}}^{2}+\nu\left\|\Lambda \Delta_{q} d\right\|_{L^{2}}^{2}-2\left(\Lambda \Delta_{q} c \mid \Delta_{q} d\right) \\
= & \left(\nu \Lambda \Delta_{q} F \mid \nu \Lambda \Delta_{q} c\right)+2\left(\Delta_{q} d \mid \Delta_{q} G\right)-\left(\nu \Lambda \Delta_{q} c \mid \Delta_{q} G\right)-\left(\nu \Lambda \Delta_{q} F \mid \Delta_{q} d\right) \\
& -\left(\nu \Lambda \Delta_{q}\left(T_{u^{j}} \partial_{j} c\right) \mid \nu \Lambda \Delta_{q} c\right)-2\left(\Delta_{q}\left(T_{u^{j}} \partial_{j} d\right) \mid \Delta_{q} d\right) \\
& +\left(\nu \Lambda \Delta_{q}\left(T_{u^{j}} \partial_{j} c\right) \mid \Delta_{q} d\right)+\left(\nu \Lambda \Delta_{q} c \mid \Delta_{q}\left(T_{u^{j}} \partial_{j} d\right)\right) .
\end{aligned}
$$

We use a very rough bound from below for the left-hand side (which amounts to loosing the smoothing effect of $\left(L P H^{1}\right)$ on $\left.d\right)$ :

$$
\left\|\nu \Lambda \Delta_{q} d\right\|_{L^{2}}^{2}-2\left\|\Delta_{q} d\right\|_{L^{2}}^{2} \geqslant \frac{7}{9}\left\|\Delta_{q} d\right\|_{L^{2}}^{2} .
$$

The forcing terms in the right-hand side are bounded by $C f_{q}\left(\left\|\nu \Lambda \Delta_{q} F\right\|_{L^{2}}+\left\|\Delta_{q} G\right\|_{L^{2}}\right)$.

On the other hand, using again Lemma 7.5 in the appendix, we get

$$
\begin{aligned}
& \left|\left(\nu \Lambda \Delta_{q}\left(T_{u^{j}} \partial_{j} c\right) \mid \nu \Lambda \Delta_{q} c\right)\right| \\
& \quad \lesssim\left\|S_{q-1} \operatorname{div} u\right\|_{L^{\infty}}\left\|\nu \Lambda \Delta_{q} c\right\|_{L^{2}}^{2}+\|\nabla u\|_{L^{\infty}}\left\|\nu \Lambda \Delta_{q} c\right\|_{L^{2}} \sum_{\left|q^{\prime}-q\right| \leqslant 3}\left\|\nu \Lambda \Delta_{q^{\prime}} c\right\|_{L^{2}},
\end{aligned}
$$




$$
\begin{aligned}
& \left|\left(\Delta_{q}\left(T_{u^{j}} \partial_{j} d\right) \mid \Delta_{q} d\right)\right| \\
& \quad \lesssim\left\|S_{q-1} \operatorname{div} u\right\|_{L^{\infty}}\left\|\Delta_{q} d\right\|_{L^{2}}^{2}+\|\nabla u\|_{L^{\infty}}\left\|\Delta_{q} d\right\|_{L^{2}} \sum_{\left|q^{\prime}-q\right| \leqslant 3}\left\|\Delta_{q^{\prime}} d\right\|_{L^{2}}, \\
& \left|\left(\nu \Lambda \Delta_{q} c \mid \Delta_{q}\left(T_{u^{j}} \partial_{j} d\right)\right)+\left(\nu \Lambda \Delta_{q}\left(T_{u^{j}} \partial_{j} c\right) \mid \Delta_{q} d\right)\right| \\
& \quad \lesssim\left\|S_{q-1} \operatorname{div} u\right\|_{L^{\infty}}\left\|\nu \Lambda \Delta_{q} c\right\|_{L^{2}}\left\|\Delta_{q} d\right\|_{L^{2}} \\
& \quad+\|\nabla u\|_{L^{\infty}}\left(\left\|\nu \Lambda \Delta_{q} c\right\|_{L^{2}} \sum_{\left|q^{\prime}-q\right| \leqslant 3}\left\|\Delta_{q^{\prime}} d\right\|_{L^{2}}+\left\|\Delta_{q} d\right\|_{L^{2}} \sum_{\left|q^{\prime}-q\right| \leqslant 3}\left\|\nu \Lambda \Delta_{q^{\prime}} c\right\|_{L^{2}}\right) .
\end{aligned}
$$

Choosing an appropriate constant $\kappa>0$, this leads to

(29) $\frac{1}{2} \frac{\mathrm{d}}{\mathrm{d} t} f_{q}^{2}+\kappa \nu^{-1} k_{q} f_{q} \leqslant C f_{q}\left(\left\|\nu \Lambda \Delta_{q} F\right\|_{L^{2}}+\left\|\Delta_{q} G\right\|_{L^{2}}+\|\nabla u\|_{L^{\infty}} \sum_{\left|q^{\prime}-q\right| \leqslant 3} k_{q^{\prime}}\right)$.

We then conclude to (24) thanks to the following Young inequality:

$$
\|\nabla u\|_{L^{\infty}} \leqslant\left(\frac{1}{r} \frac{K \nu}{\kappa}\right)^{r-1}\|\nabla u\|_{L^{\infty}}^{r}+\frac{(r-1) \kappa}{r \nu K} .
$$

\section{Third step: $L^{1}$ decay for $c$}

We are going to show that inequality (24) provides us with decay estimates for $c$ and $d$. We postpone the proof of smoothing properties for $d$ with gain of two derivatives to the next step. Rewriting (24) in terms of $h_{q}^{2}=f_{q}^{2}+\delta^{2}$ (for any $\delta>0$ ), one can divide both sides by $h_{q}$ and perform a time integration. After having $\delta$ tend to 0 , this leads to

$$
\begin{aligned}
& f_{q}(t)+\kappa \nu \min \left(2^{2 q}, \nu^{-2}\right) \int_{0}^{t} k_{q}(\tau) \mathrm{d} \tau \\
& \leqslant f_{q}(0)+C \int_{0}^{t}\left(\max \left(1,2^{q} \nu\right)\left\|\Delta_{q} F\right\|_{L^{2}}+\left\|\Delta_{q} G\right\|_{L^{2}}\right) \mathrm{d} \tau \\
& \quad+\frac{\kappa \nu}{K} \min \left(2^{2 q}, \nu^{-2}\right) \sum_{\left|q^{\prime}-q\right| \leqslant 3} \int_{0}^{t} k_{q^{\prime}}(\tau) \mathrm{d} \tau \\
& \quad+C \int_{0}^{t}\left[\left(\frac{K}{\kappa \nu}\right)^{p-1}\|\nabla u(\tau)\|_{B_{\infty}^{2 / p-2}}^{p}+\left(\frac{\nu K}{\kappa}\right)^{r-1}\|\nabla u(\tau)\|_{L^{\infty}}^{r}\right] \sum_{\left|q^{\prime}-q\right| \leqslant 3} k_{q^{\prime}} \mathrm{d} \tau .
\end{aligned}
$$

Multiply both sides by $2^{q(s-1)}$. Using (23) and summing on $\mathbb{Z}$, we infer that

$$
\begin{aligned}
& \|c(t)\|_{\widetilde{B}_{\nu}^{s, \infty}}+\|d(t)\|_{B_{2}^{s-1}}+\kappa \nu \sum_{q \in \mathbb{Z}} \int_{0}^{t} \min \left(2^{2 q}, \nu^{-2}\right) 2^{q(s-1)} k_{q}(\tau) \mathrm{d} \tau \\
& \quad \leqslant C\left(\left\|c_{0}\right\|_{\widetilde{B}_{\nu}^{s, \infty}}+\left\|d_{0}\right\|_{B_{2}^{s-1}}+\int_{0}^{t}\left(\|F(\tau)\|_{\widetilde{B}_{\nu}^{s, \infty}}+\|G(\tau)\|_{B_{2}^{s-1}}\right) \mathrm{d} \tau\right)
\end{aligned}
$$




$$
\begin{aligned}
& +\frac{C \kappa \nu}{K} \sum_{q \in \mathbb{Z}} \int_{0}^{t} \min \left(2^{2 q}, \nu^{-2}\right) 2^{q(s-1)} k_{q}(\tau) \mathrm{d} \tau+C \int_{0}^{t}\left[\left(\frac{K}{\kappa \nu}\right)^{p-1}\|\nabla u(\tau)\|_{B_{\infty, \infty}^{2 / p-2}}^{p} \|{ }^{r-1}\right. \\
& \left.+\left(\frac{\nu K}{\kappa}\right)^{r-1}\|\nabla u(\tau)\|_{L^{\infty}}^{r}\right]\left(\|c(\tau)\|_{\widetilde{B}_{\nu}^{s, \infty}}+\|d(\tau)\|_{B_{2}^{s-1}}\right) \mathrm{d} \tau .
\end{aligned}
$$

Choosing $K=2 C$ and denoting $d_{B F} \stackrel{\text { def }}{=} \sum_{q<q_{0}} \Delta_{q} d$, we eventually obtain

$$
\begin{aligned}
& \|c(t)\|_{\widetilde{B}_{\nu}^{s, \infty}}+\|d(t)\|_{B_{2}^{s-1}}+\nu \int_{0}^{t}\left(\|c(\tau)\|_{\widetilde{B}_{\nu}^{s, 1}}+\left\|d_{B F}(\tau)\right\|_{B_{2}^{s+1}}\right) \mathrm{d} \tau \\
& \lesssim\left\|c_{0}\right\|_{\widetilde{B}_{\nu}^{s, \infty}}+\left\|d_{0}\right\|_{B_{2}^{s-1}}+\int_{0}^{t}\left(\|F(\tau)\|_{\widetilde{B}_{\nu}^{s, \infty}}+\|G(\tau)\|_{B_{2}^{s-1}}\right) \mathrm{d} \tau \\
& \quad+\int_{0}^{t}\left(V_{\nu, 1}^{p, r}\right)^{\prime}(\tau)\left(\|c(\tau)\|_{\widetilde{B}_{\nu}^{s, \infty}}+\|d(\tau)\|_{B_{2}^{s-1}}\right) \mathrm{d} \tau .
\end{aligned}
$$

Now, thanks to Gronwall inequality, we conclude that

$$
\begin{aligned}
& \|c(t)\|_{\widetilde{B}_{\nu}^{s, \infty}}+\|d(t)\|_{B_{2}^{s-1}}+\nu \int_{0}^{t}\left(\|c(\tau)\|_{\widetilde{B}_{\nu}^{s, 1}}+\left\|d_{B F}(\tau)\right\|_{B_{2}^{s+1}}\right) \mathrm{d} \tau \\
& \leqslant C \mathrm{e}^{C V_{\nu, 1}^{p, r}(t)}\left(\left\|c_{0}\right\|_{\widetilde{B}_{\nu}^{s, \infty}}+\left\|d_{0}\right\|_{B_{2}^{s-1}}+\int_{0}^{t} \mathrm{e}^{-C V_{\nu, 1}^{p, r}(\tau)}\left(\|F(\tau)\|_{\widetilde{B}_{\nu}^{s, \infty}}\right.\right. \\
& \left.\left.\quad+\|G(\tau)\|_{B_{2}^{s-1}}\right) \mathrm{~d} \tau\right) .
\end{aligned}
$$

\section{Fourth step: the $L^{1}$ smoothing effect for $d$}

To achieve the proof of Proposition 4.1, we still have to show that $d_{H F} \stackrel{\text { def }}{=} \sum_{q \geqslant q_{0}} \Delta_{q} d$ satisfies

$$
\begin{aligned}
\nu \int_{0}^{t}\left\|d_{H F}(\tau)\right\|_{B_{2}^{s+1}} \mathrm{~d} \tau \leqslant & C \mathrm{e}^{C V_{\nu, 1}^{p, r}(t)}\left(\left\|c_{0}\right\|_{\widetilde{B}_{\nu}^{s, \infty}}+\left\|d_{0}\right\|_{B_{2}^{s-1}}+\int_{0}^{t} \mathrm{e}^{-C V_{\nu, 1}^{p, r}(\tau)}\left(\|F(\tau)\|_{\widetilde{B}_{\nu}^{s, \infty}}\right.\right. \\
& \left.\left.+\|G(\tau)\|_{B_{2}^{s-1}}\right) \mathrm{~d} \tau\right) .
\end{aligned}
$$

From the first equation of $\left(L P H_{q}\right)$, we get

$$
\begin{aligned}
& \frac{1}{2} \frac{\mathrm{d}}{\mathrm{d} t}\left\|\Delta_{q} d\right\|_{L^{2}}^{2}+\kappa \nu 2^{2 q}\left\|\Delta_{q} d\right\|_{L^{2}}^{2} \\
& \quad \leqslant\left\|\Delta_{q} d\right\|_{L^{2}}\left(\left\|\Delta_{q} \Lambda c\right\|_{L^{2}}+\left\|\Delta_{q} G\right\|_{L^{2}}\right)+\left|\left(\Delta_{q} d \mid \Delta_{q}\left(T_{u^{j}} \partial_{j} d\right)\right)\right| .
\end{aligned}
$$

The last term may be bounded thanks to (27). Using Young inequality and integrating in time, this yields 


$$
\begin{aligned}
& \left\|\Delta_{q} d(t)\right\|_{L^{2}}+\kappa \nu \int_{0}^{t} 2^{2 q}\left\|\Delta_{q} d(\tau)\right\|_{L^{2}} \mathrm{~d} \tau \\
& \leqslant\left\|\Delta_{q} d_{0}\right\|_{L^{2}}+C \int_{0}^{t}\left(2^{q}\left\|\Delta_{q} c\right\|_{L^{2}}+\left\|\Delta_{q} G\right\|_{L^{2}}\right) \mathrm{d} \tau \\
& +\frac{\kappa \nu}{K} 2^{2 q} \sum_{\left|q^{\prime}-q\right| \leqslant 3} \int_{0}^{t}\left\|\Delta_{q^{\prime}} d(\tau)\right\|_{L^{2}} \mathrm{~d} \tau \\
& \quad+C \int_{0}^{t}\left(\frac{K}{\kappa \nu}\right)^{p-1}\|\nabla u(\tau)\|_{B_{\infty}^{2 / p-2}}^{p} \sum_{\left|q^{\prime}-q\right| \leqslant 3}\left\|\Delta_{q^{\prime}} d(\tau)\right\|_{L^{2}} \mathrm{~d} \tau .
\end{aligned}
$$

Multiply both sides of the above inequality by $2^{q(s-1)}$ and sum for $q \geqslant q_{0}$. Choosing $K$ suitably large, we eventually get

$$
\begin{aligned}
& \left\|d_{H F}(t)\right\|_{B_{2}^{s-1}}+\nu \int_{0}^{t}\left\|d_{H F}(\tau)\right\|_{B_{2}^{s+1}} \mathrm{~d} \tau \\
& \lesssim\left\|d_{0, H F}\right\|_{B_{2}^{s-1}}+\int_{0}^{t} \nu^{1-p}\|\nabla u\|_{B_{\infty}^{2 / p-2}}^{p}\|d(\tau)\|_{B_{2}^{s-1}} \mathrm{~d} \tau \\
& \quad+\int_{0}^{t}\left(\left\|c_{H F}(\tau)\right\|_{B_{2}^{s}}+\left\|G_{H F}(\tau)\right\|_{B_{2}^{s-1}}+\left\|d_{B F}(\tau)\right\|_{B_{2}^{s+1}}\right) \mathrm{d} \tau .
\end{aligned}
$$

Plugging inequality (32) in (34), we get (33).

We conclude this section by stating a result in the same spirit for the paralinearized incompressible Navier-Stokes equation. This result will be at the root of an explosion criterion given in Section 6. Here is the precise statement:

Proposition 4.2. - Let $s \in \mathbb{R}, 1 \leqslant p<+\infty$ and $v$ be a solution of

$$
\left\{\begin{array}{l}
\partial_{t} v+\mathcal{P}\left(T_{u} \cdot \nabla v\right)-\mu \Delta v=F \\
\left.v\right|_{t=0}=v_{0}
\end{array}\right.
$$

with $\operatorname{div} v_{0}=\operatorname{div} F=0$. Then there exist a universal constant $\kappa$ and a constant $C$ depending only on $N, p$ and $s$, and such that the following estimate holds:

$$
\|v(t)\|_{B_{2}^{s-1}}+\kappa \mu \int_{0}^{t}\|v(\tau)\|_{B_{2}^{s+1}} \mathrm{~d} \tau \leqslant \mathrm{e}^{C V_{\mu}^{p}(t)}\left(\left\|v_{0}\right\|_{B_{2}^{s-1}}+\int_{0}^{t} \mathrm{e}^{-C V_{\mu}^{p}(\tau)}\|F(\tau)\|_{B_{2}^{s-1}} \mathrm{~d} \tau\right)
$$

where $V_{\mu}^{1}(t) \stackrel{\text { def }}{=} \int_{0}^{t}\|\nabla u(\tau)\|_{L^{\infty}} \mathrm{d} \tau$ and $V_{\mu}^{p}(t) \stackrel{\text { def }}{=} \int_{0}^{t} \mu^{1-p}\|\nabla u(\tau)\|_{B_{\infty, \infty}^{2 / p-2}}^{p} \mathrm{~d} \tau$ if $p>1$.

The proof (which is left to the reader) goes along the lines of the proof of Proposition 4.1. It is in fact much simpler since there is only a parabolic equation to treat. 


\section{The case of large initial data}

To prove Theorem 2.5, we are going to follow the five steps described in Section 2. In the first three steps which are devoted to a priori estimates, we will often make the distinction between the case $N \geqslant 4$ (which actually is easier) and the case $N=2,3$. This is mainly because in high dimension, more dispersive inequalities are available than in dimension $N=2,3$ (see Proposition 2.2).

Throughout the first four steps, we shall make the implicit assumption that $\left(b^{\varepsilon}, u^{\varepsilon}\right)$ is a given solution of $\left(N S C^{\varepsilon}\right)$ which belongs to $E_{\varepsilon \nu, T}^{N / 2} \cap E_{\varepsilon \nu, T}^{N / 2+\alpha}$ (for a $\left.\left.\alpha \in\right] 0,1 / 2\right]$ if $\left.N \geqslant 4, \alpha \in\right] 0,1 / 2[$ if $N=3$ and $\alpha \in] 0,1 / 6]$ if $N=2$ ) and satisfies $\left\|\varepsilon b^{\varepsilon}\right\|_{L^{\infty}} \leqslant 3 / 4$. We shall likewise assume that the corresponding incompressible solution $v$ belongs to $F_{T_{0}}^{N / 2} \cap F_{T_{0}}^{N / 2+\alpha}$.

Let us introduce some notations. First of all, we are going to drop all the exponents $\varepsilon$, and $w$ will stand for $\mathcal{P} u-v$. For $\beta \in[0,1 / 2]$, we denote

$$
\begin{aligned}
& X_{\beta}(T) \stackrel{\text { def }}{=}\|b\|_{L_{T}^{1}\left(\widetilde{B}_{\varepsilon \nu}^{N / 2+\beta, 1}\right)}+\|\mathcal{Q} u\|_{L_{T}^{1}\left(B_{2}^{N / 2+1+\beta}\right)}+\|b\|_{L_{T}^{\infty}\left(\widetilde{B}_{\varepsilon \nu}^{N / 2+\beta, \infty}\right)}+\|\mathcal{Q} u\|_{L_{T}^{\infty}\left(B_{2}^{N / 2-1+\beta}\right)}, \\
& V_{\beta}(T) \stackrel{\text { def }}{=}\|v\|_{L_{T}^{1}\left(B_{2}^{N / 2+1+\beta}\right)}+\|v\|_{L_{T}^{\infty}\left(B_{2}^{N / 2-1+\beta}\right)}, \\
& W_{\beta}(T) \stackrel{\text { def }}{=}\|w\|_{L_{T}^{1}\left(B_{2}^{N / 2+1+\beta}\right)}+\|w\|_{L_{T}^{\infty}\left(B_{2}^{N / 2-1+\beta}\right)}, \\
& Y_{\beta}(T) \stackrel{\text { def }}{=}\|b\|_{L_{T}^{2}\left(B_{\infty}^{\beta-1 / 2}\right)}+\|\mathcal{Q} u\|_{L_{T}^{2}\left(B_{\infty}^{\beta-1 / 2}\right)} \quad \text { if } N \geqslant 4, \\
& Y_{\beta}^{p}(T) \stackrel{\text { def }}{=}\|b\|_{L_{T}^{p}\left(B_{\infty}^{\beta-1+1 / p}\right)}+\|\mathcal{Q} u\|_{L_{T}^{p}\left(B_{\infty}^{\beta-1+1 / p}\right)} \quad \text { if } N=3 \text { and } 2<p \leqslant+\infty, \\
& Y_{\beta}(T) \stackrel{\text { def }}{=}\|b\|_{L_{T}^{4}\left(B_{\infty}^{\beta-3 / 4}\right)}+\|\mathcal{Q} u\|_{L_{T}^{4}\left(B_{\infty}^{\beta-3 / 4}\right)} \quad \text { if } N=2 .
\end{aligned}
$$

We shall also use the notation $P_{\beta}(T)=V_{\beta}(T)+W_{\beta}(T)$ and it is meant that

$$
X_{\beta}^{0}=\left\|b_{0}\right\|_{\widetilde{B}_{\varepsilon \nu}^{N / 2+\beta, \infty}}+\left\|\mathcal{Q} u_{0}\right\|_{B_{2}^{N / 2-1+\beta}}+\|\mathcal{Q} f\|_{L^{1}\left(B_{2}^{N / 2-1+\beta}\right)} .
$$

In absence of ambiguity, the $T$ will be omitted, and $\beta$ will always stand for 0 or $\alpha$.

Now, let us tackle the first step of the proof.

First step: Dispersive estimates for $(b, \mathcal{Q} u)$

Denote

$$
G \stackrel{\text { def }}{=} \nu \Delta \mathcal{Q} u-\mathcal{Q}\left(u \cdot \nabla u-\frac{\varepsilon b}{1+\varepsilon b} \mathcal{A} u+\frac{K(\varepsilon b) b}{\varepsilon}\right) .
$$

Applying Proposition 2.2 to the first two equations of (10), we get for $N \geqslant 4$,

$$
\begin{aligned}
Y_{\alpha} \lesssim & \varepsilon^{1 / 2}\left(\left\|\left(b_{0}, \mathcal{Q} u_{0}\right)\right\|_{B_{2}^{N / 2-1+\alpha}}+\|\mathcal{Q} f\|_{L_{T}^{1}\left(B_{2}^{N / 2-1+\alpha}\right)}+\|\operatorname{div}(b u)\|_{L_{T}^{1}\left(B_{2}^{N / 2-1+\alpha}\right)}\right. \\
& \left.+\|G\|_{L_{T}^{1}\left(B_{2}^{N / 2-1+\alpha}\right)}\right)
\end{aligned}
$$

for $N=3$ and $2<p \leqslant+\infty$,

$$
\begin{aligned}
Y_{\alpha}^{p} \lesssim & \varepsilon^{1 / p}\left(\left\|\left(b_{0}, \mathcal{Q} u_{0}\right)\right\|_{B_{2}^{1 / 2+\alpha}}+\|\mathcal{Q} f\|_{L_{T}^{1}\left(B_{2}^{1 / 2+\alpha}\right)}\right. \\
& \left.+\|\operatorname{div}(b u)\|_{L_{T}^{1}\left(B_{2}^{1 / 2+\alpha}\right)}+\|G\|_{L_{T}^{1}\left(B_{2}^{1 / 2+\alpha}\right)}\right)
\end{aligned}
$$


for $N=2$,

$$
Y_{\alpha} \lesssim \varepsilon^{1 / 4}\left(\left\|\left(b_{0}, \mathcal{Q} u_{0}\right)\right\|_{B_{2}^{\alpha}}+\|\mathcal{Q} f\|_{L_{T}^{1}\left(B_{2}^{\alpha}\right)}+\|\operatorname{div}(b u)\|_{L_{T}^{1}\left(B_{2}^{\alpha}\right)}+\|G\|_{L_{T}^{1}\left(B_{2}^{\alpha}\right)}\right) .
$$

From Proposition 1.4, Lemma 1.5 and (7), we easily gather that for any $N \geqslant 2$,

$$
\begin{aligned}
& \|\operatorname{div}(b u)\|_{L_{T}^{1}\left(B_{2}^{N / 2-1+\alpha}\right)} \lesssim\|b\|_{L_{T}^{2}\left(B_{2}^{N / 2}\right)}\|u\|_{L_{T}^{2}\left(B_{2}^{N / 2+\alpha}\right)}+\|u\|_{L_{T}^{2}\left(B_{2}^{N / 2}\right)}\|b\|_{L_{T}^{2}\left(B_{2}^{N / 2+\alpha}\right)}, \\
& \lesssim X_{0}\left(X_{\alpha}+P_{\alpha}\right)+X_{\alpha}\left(X_{0}+P_{0}\right) \\
& \|\mathcal{Q}(u \cdot \nabla u)\|_{L_{T}^{1}\left(B_{2}^{N / 2-1+\alpha}\right)} \lesssim\|u\|_{L_{T}^{2}\left(B_{2}^{N / 2}\right)}\|\nabla u\|_{L_{T}^{2}\left(B_{2}^{N / 2-1+\alpha}\right)}, \\
& \lesssim\left(X_{0}+P_{0}\right)\left(X_{\alpha}+P_{\alpha}\right) \text {, } \\
& \left\|\mathcal{Q}\left(\frac{\varepsilon b}{1+\varepsilon b} \mathcal{A} u\right)\right\|_{L_{T}^{1}\left(B_{2}^{N / 2-1+\alpha}\right)} \lesssim \varepsilon\|b\|_{L_{T}^{\infty}\left(B_{2}^{N / 2}\right)}\|\mathcal{A} u\|_{L_{T}^{1}\left(B_{2}^{N / 2-1+\alpha}\right)}, \\
& \lesssim X_{0}\left(X_{\alpha}+P_{\alpha}\right) \\
& \left\|\mathcal{Q}\left(\frac{K(\varepsilon b) b}{\varepsilon}\right)\right\|_{L_{T}^{1}\left(B_{2}^{N / 2-1+\alpha}\right)} \lesssim \varepsilon^{-1}\|\varepsilon b\|_{L_{T}^{2}\left(B_{2}^{N / 2}\right)}\|\nabla b\|_{L_{T}^{2}\left(B_{2}^{N / 2-1+\alpha}\right)}, \\
& \lesssim X_{0} X_{\alpha} .
\end{aligned}
$$

Plugging the above inequalities in (35), (36) or (37), we conclude that

$$
\begin{cases}Y_{\alpha} \lesssim \varepsilon^{1 / 2}\left(X_{\alpha}^{0}+X_{\alpha}+\left(X_{0}+P_{0}\right)\left(X_{\alpha}+P_{\alpha}\right)\right) & \text { if } N \geqslant 4 \\ Y_{\alpha}^{p} \lesssim \varepsilon^{1 / p}\left(X_{\alpha}^{0}+X_{\alpha}+\left(X_{0}+P_{0}\right)\left(X_{\alpha}+P_{\alpha}\right)\right) & \text { if } N=3 \text { and } 2<p \leqslant+\infty \\ Y_{\alpha} \lesssim \varepsilon^{1 / 4}\left(X_{\alpha}^{0}+X_{\alpha}+\left(X_{0}+P_{0}\right)\left(X_{\alpha}+P_{\alpha}\right)\right) & \text { if } N=2\end{cases}
$$

Remark 5.1. - It goes without saying that Proposition 2.2 actually provides us with estimates for $(b, \mathcal{Q} u)$ in a plethora of spaces $L_{T}^{r}\left(B_{p}^{\sigma}\right)$.

Second step: Estimates for $w$ in $L^{1}\left(0, T ; B_{2}^{N / 2+1+\beta}\right) \cap C\left([0, T] ; B_{2}^{N / 2-1+\beta}\right)$

From the third equation of (10) and (NSI), we gather that

$$
\partial_{t} w+\mathcal{P}(A \cdot \nabla w)+\mathcal{P}(w \cdot \nabla B)-\mu \Delta w=\mathcal{P} F
$$

with $A=B=\mathcal{Q} u+v$ and $F=-\left(\mathcal{Q} u \cdot \nabla v+v \cdot \nabla \mathcal{Q} u+w \cdot \nabla w+\frac{\varepsilon b}{1+\varepsilon b} \mathcal{A} u\right)$.

Apply Proposition 7.4 with $s=N / 2+\beta$. This yields

$$
W_{\beta} \lesssim \mathrm{e}^{C\left(V_{0}+X_{0}\right)}\|F\|_{L_{T}^{1}\left(B_{2}^{N / 2-1+\beta}\right)} .
$$

For the last term in $F$, we readily have

$$
\|w \cdot \nabla w\|_{L_{T}^{1}\left(B_{2}^{N / 2-1+\beta}\right)} \lesssim\|w\|_{L_{T}^{2}\left(B_{2}^{N / 2}\right)}\|\nabla w\|_{L_{T}^{2}\left(B_{2}^{N / 2-1+\beta}\right)} \lesssim W_{0} W_{\beta} .
$$

Next, by interpolation and according to (7), we have

$$
\|b\|_{B_{2}^{N / 2}} \leqslant\|b\|_{B_{2}^{N / 2+\alpha-1}}^{\alpha}\|b\|_{B_{2}^{N / 2+\alpha}}^{1-\alpha} \leqslant(\varepsilon \nu)^{\alpha-1}\|b\|_{\widetilde{B}_{\varepsilon \nu}^{N / 2+\alpha, \infty}} .
$$


From it we deduce that

$$
\begin{aligned}
\left\|\frac{\varepsilon b}{1+\varepsilon b} \mathcal{A} u\right\|_{L_{T}^{1}\left(B_{2}^{N / 2-1+\beta}\right)} & \lesssim \varepsilon\|b\|_{L_{T}^{\infty}\left(B_{2}^{N / 2}\right)}\|\mathcal{A} u\|_{L_{T}^{1}\left(B_{2}^{N / 2-1+\beta}\right)}, \\
& \lesssim \varepsilon^{\alpha}\|b\|_{L_{T}^{\infty}\left(\widetilde{B}_{\varepsilon \nu}^{N / 2+\alpha, \infty}\right)}\|u\|_{L_{T}^{1}\left(B_{2}^{N / 2+1+\beta}\right)} \\
& \lesssim \varepsilon^{\alpha} X_{\alpha}\left(V_{\beta}+W_{\beta}+X_{\beta}\right) .
\end{aligned}
$$

The two remaining terms will be treated differently according to $N \geqslant 3$ or $N=2$.

Case $N \geqslant 3$ : According to step one, $\mathcal{Q} u$ is small in $L^{2}\left(0, T ; B_{\infty}^{0}\right)$. Indeed, using interpolation and injection (see Proposition 1.3(iii) and (v)), we have if $N \geqslant 4$

$$
\begin{aligned}
\|\mathcal{Q} u\|_{L_{T}^{2}\left(B_{\infty}^{0}\right)} & \leqslant\|\mathcal{Q} u\|_{L_{T}^{2}\left(B_{\infty}^{\alpha-1 / 2}\right)}^{2 \alpha}\|\mathcal{Q} u\|_{L_{T}^{2}\left(B_{\infty}^{\alpha}\right)}^{1-2 \alpha} \\
& \lesssim \varepsilon^{\alpha}\left(\varepsilon^{-1 / 2}\|\mathcal{Q} u\|_{L_{T}^{2}\left(B_{\infty}^{\alpha-1 / 2}\right)}\right)^{2 \alpha}\|\mathcal{Q} u\|_{L_{T}^{2}\left(B_{2}^{N / 2+\alpha}\right)}^{1-2 \alpha} \\
& \lesssim \varepsilon^{\alpha}\left(X_{\alpha}+\varepsilon^{-1 / 2} Y_{\alpha}\right)
\end{aligned}
$$

and if $N=3$ and $p_{\alpha}=1+1 / 2 \alpha$,

$$
\begin{aligned}
\|\mathcal{Q} u\|_{L_{T}^{2}\left(B_{\infty}^{0}\right)} & \leqslant\|\mathcal{Q} u\|_{L_{T}^{1}\left(B_{\infty}^{1+\alpha}\right)}^{1 / 2-\alpha}\|\mathcal{Q} u\|_{L_{T}^{(1+2 \alpha) / 2 \alpha}\left(B_{\infty}^{\alpha-1 /(1+2 \alpha)}\right)}^{1 / 2+\alpha} \\
& \lesssim\|\mathcal{Q} u\|_{L_{T}^{1}\left(B_{2}^{5 / 2+\alpha}\right)}^{1 / 2-\alpha}\|\mathcal{Q} u\|_{L_{T}^{(1+2 \alpha) / 2 \alpha}\left(B_{\infty}^{\alpha-1 /(1+2 \alpha)}\right)}^{1 / 2+\alpha} \\
& \lesssim \varepsilon^{\alpha}\left(X_{\alpha}+\varepsilon^{-2 \alpha /(1+2 \alpha)} Y_{\alpha}^{p_{\alpha}}\right)
\end{aligned}
$$

From (43) and (44) we expect to glean some smallness for $\mathcal{Q} u \cdot \nabla v$ and $v \cdot \nabla \mathcal{Q} u$. A judicious application of paradifferential calculus will enable us to get it. For $\mathcal{Q} u \cdot \nabla v$, we shall use the following decomposition (with $\eta<1$ to be fixed hereafter)

$$
Q u \cdot \nabla v=\underbrace{\sum_{q \in \mathbb{Z}} \Delta_{q} \mathcal{Q} u \cdot S_{q-1+\left[\log _{2} \eta\right]} \nabla v}_{T_{1}}+\underbrace{\sum_{q \in \mathbb{Z}} S_{q+2-\left[\log _{2} \eta\right]} \mathcal{Q} u \cdot \Delta_{q} \nabla v}_{T_{2}}
$$

which may be seen as a slight modification of Bony decomposition.

Let us remark that for any $j \in \mathbb{Z}$, we have

$$
\left\|S_{j} \nabla v\right\|_{L^{\infty}} \lesssim 2^{2 j}\|\nabla v\|_{B_{\infty}^{-2}}
$$

Therefore

$$
\begin{aligned}
\left\|S_{q-1+\left[\log _{2} \eta\right]} \nabla v \Delta_{q} \mathcal{Q} u\right\|_{L^{2}} & \lesssim\left\|S_{q-1+\left[\log _{2} \eta\right]} \nabla v\right\|_{L^{\infty}}\left\|\Delta_{q} \mathcal{Q} u\right\|_{L^{2}}, \\
& \lesssim \eta^{2} 2^{-q(N / 2+\beta-1)}\|\nabla v\|_{B_{\infty}^{-2}}\left(2^{q(N / 2+\beta+1)}\left\|\Delta_{q} \mathcal{Q} u\right\|_{L^{2}}\right) .
\end{aligned}
$$

Since, according to (4), the function $\mathcal{F}\left(S_{q-1+\left[\log _{2} \eta\right]} \nabla v \Delta_{q} \mathcal{Q} u\right)$ is supported in a dyadic annulus $2^{q} C\left(0, R_{1}, R_{2}\right)$ with $R_{1}$ and $R_{2}$ independent of $\eta$, Lemma 1.2 yields

$$
\left\|T_{1}\right\|_{L_{T}^{1}\left(B_{2}^{N / 2-1+\beta}\right)} \lesssim \eta^{2}\|\nabla v\|_{L_{T}^{\infty}\left(B_{2}^{N / 2-2}\right)}\|\mathcal{Q} u\|_{L_{T}^{1}\left(B_{2}^{N / 2+1+\beta}\right)} .
$$

On the other hand, according to (4), we have for all $p \in \mathbb{Z}$ :

$$
\Delta_{p} T_{2}=\sum_{q \geqslant p-2+\left[\log _{2} \eta\right]} \Delta_{p}\left(S_{q+2-\left[\log _{2} \eta\right]} \mathcal{Q} u \cdot \Delta_{q} \nabla v\right) .
$$


Therefore

$$
\begin{aligned}
2^{p(N / 2+\beta-1)}\left\|\Delta_{p} T_{2}\right\|_{L^{2}} & \lesssim 2^{p(N / 2+\beta-1)}\|\mathcal{Q} u\|_{L^{\infty}} \sum_{q \geqslant p-2+\left[\log _{2} \eta\right]}\left\|\Delta_{q} \nabla v\right\|_{L^{2}} \\
& \lesssim \eta^{1-\beta-N / 2}\|\mathcal{Q} u\|_{L^{\infty}}\|\nabla v\|_{B_{2}^{N / 2+\beta-1}}
\end{aligned}
$$

whence,

$$
\left\|T_{2}\right\|_{B_{2}^{N / 2+\beta-1}} \lesssim \eta^{1-\beta-N / 2}\|v\|_{B_{2}^{N / 2+\beta}}\|\mathcal{Q} u\|_{L^{\infty}} .
$$

Choose $\eta=\varepsilon^{2 \alpha /(2+N+2 \beta)}$. Since $B_{\infty}^{0} \hookrightarrow L^{\infty}$, we conclude from (45), (46), and (43) or (44) that

$$
\begin{aligned}
& \|\mathcal{Q} u \cdot \nabla v\|_{L_{T}^{1}\left(B_{2}^{N / 2-1+\beta}\right)} \lesssim \varepsilon^{4 \alpha /(2+N+2 \beta)}\left(V_{0} X_{\beta}+V_{\beta}\left(X_{\alpha}+\varepsilon^{-1 / 2} Y_{\alpha}\right)\right) \quad \text { if } N \geqslant 4, \\
& \|\mathcal{Q} u \cdot \nabla v\|_{L_{T}^{1}\left(B_{2}^{N / 2-1+\beta}\right)} \lesssim \varepsilon^{4 \alpha /(2+N+2 \beta)}\left(V_{0} X_{\beta}+V_{\beta}\left(X_{\alpha}+\varepsilon^{-2 \alpha /(1+2 \alpha)} Y_{\alpha}^{p_{\alpha}}\right)\right) \\
& \quad \text { if } N=3 .
\end{aligned}
$$

The term $v \cdot \nabla \mathcal{Q} u$ may be treated similarly. Use the decomposition

$$
v \cdot \nabla \mathcal{Q} u=\underbrace{\sum_{q \in \mathbb{Z}} S_{q-1+\left[\log _{2} \eta\right]} v \cdot \Delta_{q} \nabla \mathcal{Q} u}_{\widetilde{T}_{1}}+\underbrace{\sum_{q \in \mathbb{Z}} \Delta_{q} v \cdot S_{q+2-\left[\log _{2} \eta\right]} \nabla \mathcal{Q} u}_{\widetilde{T}_{2}} .
$$

Following the proof of (45), we readily get

$$
\begin{aligned}
& \left\|\widetilde{T}_{1}\right\|_{L_{T}^{1}\left(B_{2}^{N / 2-1+\beta}\right)} \lesssim \eta\|v\|_{L_{T}^{\infty}\left(B_{2}^{N / 2-1}\right)}\|\nabla \mathcal{Q} u\|_{L_{T}^{1}\left(B_{2}^{N / 2+\beta}\right)}, \\
& \left\|\widetilde{T}_{2}\right\|_{L_{T}^{1}\left(B_{2}^{N / 2-1+\beta}\right)} \lesssim \eta^{-N / 2-\beta}\|v\|_{L_{T}^{2}\left(B_{2}^{N / 2-1}\right)}\|\nabla \mathcal{Q} u\|_{L_{T}^{2}\left(B_{\infty}^{-1}\right)} .
\end{aligned}
$$

Choosing $\eta=\varepsilon^{2 \alpha /(2+N+2 \beta)}$, we conclude that

$$
\begin{aligned}
& \|v \cdot \nabla \mathcal{Q} u\|_{L_{T}^{1}\left(B_{2}^{N / 2-1+\beta}\right)} \lesssim \varepsilon^{2 \alpha /(2+N+2 \beta)}\left(V_{0} X_{\beta}+V_{\beta}\left(X_{\alpha}+\varepsilon^{-1 / 2} Y_{\alpha}\right)\right) \quad \text { if } N \geqslant 4, \\
& \|v \cdot \nabla \mathcal{Q} u\|_{L_{T}^{1}\left(B_{2}^{N / 2-1+\beta}\right)} \lesssim \varepsilon^{2 \alpha /(2+N+2 \beta)}\left(V_{0} X_{\beta}+V_{\beta}\left(X_{\alpha}+\varepsilon^{-2 \alpha /(1+2 \alpha)} Y_{\alpha}^{p_{\alpha}}\right)\right) \\
& \quad \text { if } N=3 .
\end{aligned}
$$

Plugging inequalities (40), (42), (47) and (48) into (39), we eventually get, if $N \geqslant 4$

$$
\begin{aligned}
W_{\beta} \leqslant & C \mathrm{e}^{C\left(V_{0}+X_{0}\right)}\left(W_{0} W_{\beta}+\varepsilon^{\alpha} X_{\alpha}\left(V_{\beta}+X_{\beta}+W_{\beta}\right)\right. \\
& \left.+\varepsilon^{2 \alpha /(2+N+2 \beta)}\left(V_{0} X_{\beta}+V_{\beta}\left(X_{\alpha}+\varepsilon^{-1 / 2} Y_{\alpha}\right)\right)\right),
\end{aligned}
$$

and if $N=3$,

$$
\begin{aligned}
W_{\beta} \leqslant & C \mathrm{e}^{C\left(V_{0}+X_{0}\right)}\left(W_{0} W_{\beta}+\varepsilon^{\alpha} X_{\alpha}\left(V_{\beta}+X_{\beta}+W_{\beta}\right)\right. \\
& \left.+\varepsilon^{2 \alpha /(5+2 \beta)}\left(V_{0} X_{\beta}+V_{\beta}\left(X_{\alpha}+\varepsilon^{-2 \alpha /(1+2 \alpha)} Y_{\alpha}^{p_{\alpha}}\right)\right)\right) .
\end{aligned}
$$

Case $N=2$ : That case is more intricate for two reasons. First, proving an estimate of type (43) for $\mathcal{Q} u$ in $L^{2}\left(B_{\infty}^{0}\right)$ is hopeless (the reason why is that Proposition 2.2 yields estimates in spaces of type $L_{T}^{r}\left(B_{p}^{s}\right)$ with $r \geqslant 4$ only). On the other hand, the following estimate is available (recall that here $\alpha \in] 0,1 / 6]$ ): 


$$
\begin{aligned}
\|\mathcal{Q} u\|_{L_{T}^{1 /(1-3 \alpha)}\left(B_{2 /(1-4 \alpha)}^{2-10 \alpha}\right)} & \leqslant\|\mathcal{Q} u\|_{L_{T}^{1}\left(B_{2}^{\alpha+2}\right)}^{1-4 \alpha}\|\mathcal{Q} u\|_{L_{T}^{4}\left(B_{\infty}^{\alpha-3 / 4}\right)}^{4 \alpha}, \\
& \lesssim \varepsilon^{\alpha}\left(X_{\alpha}+\varepsilon^{-1 / 4} Y_{\alpha}\right),
\end{aligned}
$$

and we shall often utilize the embedding $B_{2 /(1-4 \alpha)}^{2-10 \alpha} \hookrightarrow B_{\infty}^{1-6 \alpha}$.

Next, due to the low regularity of the norms considered, we will have to be a bit more careful when writing estimates for the terms $\mathcal{Q} u \cdot \nabla v$ and $v \cdot \nabla \mathcal{Q} u$.

The key to the proof lies on the following refined Bony decomposition:

$$
\begin{aligned}
\mathcal{Q} u \cdot \nabla v= & \sum_{q \in \mathbb{Z}} S_{q-1+\left[\log _{2} \eta\right]} \mathcal{Q} u \nabla \Delta_{q} v+\sum_{q \in \mathbb{Z}}\left(S_{q-1}-S_{q-1+\left[\log _{2} \eta\right]}\right) \mathcal{Q} u \nabla \Delta_{q} v \\
& +R(\mathcal{Q} u, \nabla v)+T_{\nabla v} \mathcal{Q} u .
\end{aligned}
$$

Using arguments of case $N \geqslant 3$ and (51), we get

$$
\begin{gathered}
\left\|\sum_{q \in \mathbb{Z}} S_{q-1+\left[\log _{2} \eta\right]} \mathcal{Q} u \nabla \Delta_{q} v\right\|_{L_{T}^{1}\left(B_{2}^{\beta}\right)} \lesssim \eta\|\mathcal{Q} u\|_{L_{T}^{\infty}\left(B_{\infty}^{-1}\right)}\|\nabla v\|_{L_{T}^{1}\left(B_{2}^{1+\beta}\right)}, \\
\lesssim \eta X_{0} V_{\beta} \\
\left\|\sum_{q \in \mathbb{Z}}\left(S_{q-1}-S_{q-1+\left[\log _{2} \eta\right]}\right) \mathcal{Q} u \nabla \Delta_{q} v\right\|_{L_{T}^{1}\left(B_{2}^{\beta}\right)} \\
\lesssim \eta^{6 \alpha-1}\|\mathcal{Q} u\|_{L_{T}^{1 /(1-3 \alpha)}\left(B_{\infty}^{1-6 \alpha}\right)}\|\nabla v\|_{L_{T}^{1 /(3 \alpha)}\left(B_{2}^{6 \alpha-1+\beta}\right)} \\
\lesssim \eta^{6 \alpha-1} \varepsilon^{\alpha} V_{\beta}\left(X_{\alpha}+\varepsilon^{-1 / 4} Y_{\alpha}\right) .
\end{gathered}
$$

Whence, choosing $\eta=\varepsilon^{\alpha /(2-6 \alpha)}$,

$$
\left\|T_{\mathcal{Q} u} \nabla v\right\|_{L_{T}^{1}\left(B_{2}^{\beta}\right)} \lesssim \varepsilon^{\alpha /(2-6 \alpha)}\left(X_{0} V_{\beta}+V_{\beta}\left(X_{\alpha}+\varepsilon^{-1 / 4} Y_{\alpha}\right)\right) .
$$

Next, according to Proposition 1.9 and (51), we have

$$
\begin{aligned}
\|R(\mathcal{Q} u, \nabla v)\|_{L_{T}^{1}\left(B_{2}^{\beta}\right)} & \lesssim\|\nabla v\|_{L_{T}^{1 /(3 \alpha)}\left(B_{2}^{6 \alpha+\beta-1}\right)}\|\mathcal{Q} u\|_{L_{T}^{1 /(1-3 \alpha)}\left(B_{2 /(1-4 \alpha)}^{2-10 \alpha}\right)}, \\
& \lesssim \varepsilon^{\alpha} V_{\beta}\left(X_{\alpha}+\varepsilon^{-1 / 4} Y_{\alpha}\right) .
\end{aligned}
$$

On the other hand, since $\alpha, \beta \leqslant 1 / 6$, we have $10 \alpha+\beta-2<0$. Hence

$$
\begin{aligned}
\left\|S_{q-1} \nabla v \Delta_{q} \mathcal{Q} u\right\|_{L^{2}} & \leqslant\left\|\Delta_{q} \mathcal{Q} u\right\|_{L^{2 /(1-4 \alpha)}} \sum_{q^{\prime} \leqslant q-2}\left\|\Delta_{q^{\prime}} \nabla v\right\|_{L^{1 /(2 \alpha)}}, \\
& \leqslant 2^{-q \beta}\left(2^{q(2-10 \alpha)}\left\|\Delta_{q} \mathcal{Q} u\right\|_{L^{2 /(1-4 \alpha)}}\right)\|\nabla v\|_{B_{1 /(2 \alpha)}^{10 \alpha+\beta-2} .}
\end{aligned}
$$

Apply Lemma 1.2 and use the embedding $B_{2}^{6 \alpha+\beta-1} \hookrightarrow B_{1 /(2 \alpha)}^{10 \alpha+\beta-2}$. We get

$$
\begin{aligned}
\left\|T_{\nabla v} \mathcal{Q} u\right\|_{L_{T}^{1}\left(B_{2}^{\beta}\right)} & \lesssim\|\nabla v\|_{L_{T}^{1 /(3 \alpha)}\left(B_{2}^{6 \alpha+\beta-1}\right)}\|\mathcal{Q} u\|_{L_{T}^{1 /(1-3 \alpha)}\left(B_{2 /(1-4 \alpha)}^{2-10 \alpha}\right)}, \\
& \lesssim \varepsilon^{\alpha} V_{\beta}\left(X_{\alpha}+\varepsilon^{-1 / 4} Y_{\alpha}\right) .
\end{aligned}
$$

We shall use a similar decomposition for $v \cdot \nabla \mathcal{Q} u$ :

$$
\begin{aligned}
v \cdot \nabla \mathcal{Q} u= & T_{\nabla \mathcal{Q} u} v+R(v, \nabla \mathcal{Q} u)+\sum_{q \in \mathbb{Z}} S_{q-1+\left[\log _{2} \eta\right]} v \nabla \Delta_{q} \mathcal{Q} u \\
& +\sum_{q \in \mathbb{Z}}\left(S_{q-1}-S_{q-1+\left[\log _{2} \eta\right]}\right) v \nabla \Delta_{q} \mathcal{Q} u .
\end{aligned}
$$


According to Proposition 1.9, we have

$$
\begin{aligned}
\left\|T_{\nabla \mathcal{Q} u} v\right\|_{L_{T}^{1}\left(B_{2}^{\beta}\right)} & \lesssim\|\nabla \mathcal{Q} u\|_{L_{T}^{1 /(1-3 \alpha)}\left(B_{\infty}^{-6 \alpha}\right)}\|v\|_{L_{T}^{1 /(3 \alpha)}\left(B_{2}^{6 \alpha+\beta}\right)}, \\
& \lesssim \varepsilon^{\alpha} V_{\beta}\left(X_{\alpha}+\varepsilon^{-1 / 4} Y_{\alpha}\right), \\
\|R(\nabla \mathcal{Q} u, v)\|_{L_{T}^{1}\left(B_{2}^{\beta}\right)} & \lesssim\|\nabla \mathcal{Q} u\|_{L^{1 /(1-3 \alpha)}\left(B_{2 /(1-4 \alpha)}^{1-10 \alpha}\right)}\|v\|_{L_{T}^{1 /(3 \alpha)}\left(B_{2}^{6 \alpha+\beta}\right)}, \\
& \lesssim \varepsilon^{\alpha} V_{\beta}\left(X_{\alpha}+\varepsilon^{-1 / 4} Y_{\alpha}\right) .
\end{aligned}
$$

And, following the computations of the case $N \geqslant 3$, we have

$$
\begin{gathered}
\left\|\sum_{q \in \mathbb{Z}} S_{q-1+\left[\log _{2} \eta\right]} v \nabla \Delta_{q} \mathcal{Q} u\right\|_{L_{T}^{1}\left(B_{2}^{\beta}\right)} \\
\lesssim \eta\|v\|_{L_{T}^{\infty}\left(B_{\infty}^{-1}\right)}\|\nabla \mathcal{Q} u\|_{L_{T}^{1}\left(B_{2}^{1+\beta}\right)} \\
\lesssim \eta V_{0} X_{\beta} \\
\left\|\sum_{q \in \mathbb{Z}}\left(S_{q-1}-S_{q-1+\left[\log _{2} \eta\right]}\right) v \nabla \Delta_{q} \mathcal{Q} u\right\|_{L_{T}^{1}\left(B_{2}^{\beta}\right)} \\
\lesssim \eta^{-6 \alpha-\beta}\|v\|_{L_{T}^{1 /(3 \alpha)}\left(B_{2}^{6 \alpha+\beta}\right)}\|\nabla \mathcal{Q} u\|_{L_{T}^{1 /(1-3 \alpha)}\left(B_{\infty}^{-6 \alpha}\right)} \\
\lesssim \eta^{-6 \alpha-\beta} \varepsilon^{\alpha} V_{\beta}\left(X_{\alpha}+\varepsilon^{-1 / 4} Y_{\alpha}\right)
\end{gathered}
$$

so that, choosing $\eta=\varepsilon^{\alpha /(1+6 \alpha+\beta)}$, we get

$$
\left\|T_{v} \nabla \mathcal{Q} u\right\|_{L_{T}^{1}\left(B_{2}^{\beta}\right)} \lesssim \varepsilon^{\alpha /(1+6 \alpha+\beta)}\left(V_{0} X_{\beta}+V_{\beta}\left(X_{\alpha}+\varepsilon^{-1 / 4} Y_{\alpha}\right)\right)
$$

Plugging (40), (42) and (52)-(57) in (39), we conclude to

$$
W_{\beta} \leqslant C \mathrm{e}^{C\left(V_{0}+X_{0}\right)}
$$

$$
\times\left(W_{0} W_{\beta}+\varepsilon^{\alpha} X_{\alpha}\left(V_{\beta}+X_{\beta}+W_{\beta}\right)+\varepsilon^{\alpha /(2+\beta)}\left(V_{0} X_{\beta}+V_{\beta}\left(X_{\alpha}+\varepsilon^{-1 / 4} Y_{\alpha}\right)\right)\right) .
$$

Third step: Estimates for $(b, \mathcal{Q} u)$ in $E_{\varepsilon \nu, T}^{N / 2+\beta}$

Denote $d \stackrel{\text { def }}{=} \Lambda^{-1} \operatorname{div} \mathcal{Q} u$. From (10), we gather the following system for $(b, d)$ :

$$
\left\{\begin{array}{l}
\partial_{t} b+T_{u^{j}} \partial_{j} b+\frac{\Lambda d}{\varepsilon}=F \\
\partial_{t} d+T_{u^{j}} \partial_{j} d-\nu \Delta d-\frac{\Lambda b}{\varepsilon}=G+\Lambda^{-1} \operatorname{div} \mathcal{Q} f
\end{array}\right.
$$

with

$$
\begin{aligned}
& F \stackrel{\text { def }}{=}-T_{\partial_{j} b}^{\prime} u^{j}-b \operatorname{div} \mathcal{Q} u, \\
& G \stackrel{\text { def }}{=} \Lambda^{-1} \operatorname{div}\left(K(\varepsilon b) \frac{\nabla b}{\varepsilon}-\frac{\varepsilon b}{1+\varepsilon b} \mathcal{A} u\right)-T_{\partial_{j} \operatorname{div} \Lambda^{-1} \mathcal{Q} u}^{\prime} u^{j} \\
& +\left(u \cdot \nabla \Lambda^{-1} \operatorname{div} \mathcal{Q} u-\Lambda^{-1} \operatorname{div}(u \cdot \nabla u)\right) .
\end{aligned}
$$

According to Proposition 4.1, we have 


$$
\begin{aligned}
X_{\beta}(T) \leqslant & C \mathrm{e}^{V_{\nu, \varepsilon}^{p, r}(T)}\left(\left\|b_{0}\right\|_{\widetilde{B}_{\varepsilon \nu}^{N / 2+\beta, \infty}}+\left\|d_{0}\right\|_{B_{2}^{N / 2-1+\beta}}\right. \\
& \left.+\|\mathcal{Q} f\|_{L_{T}^{1}\left(B_{2}^{N / 2-1+\beta}\right)}+\|F\|_{L_{T}^{1}\left(\widetilde{B}_{\varepsilon \nu}^{N / 2+\beta, \infty}\right)}+\|G\|_{L_{T}^{1}\left(B_{2}^{N / 2-1+\beta}\right)}\right)
\end{aligned}
$$

with

$$
V_{\nu, \varepsilon}^{p, r}(t) \stackrel{\text { def }}{=} \int_{0}^{t}\left(\nu^{1-p}\|\nabla u(\tau)\|_{B_{\infty}^{2 / p-2}}^{p}+\left(\varepsilon^{2} \nu\right)^{r-1}\|\nabla u(\tau)\|_{L^{\infty}}^{r}\right) \mathrm{d} \tau
$$

for any $p, r>1$ (to be fixed hereafter).

\section{Estimates for $F$}

According to (7),

$$
\|F\|_{L_{T}^{1}\left(\widetilde{B}_{\varepsilon \nu}^{N / 2+\beta, \infty}\right)} \lesssim\|F\|_{L_{T}^{1}\left(B_{2}^{N / 2-1+\beta}\right)}+\varepsilon \nu\|F\|_{L_{T}^{1}\left(B_{2}^{N / 2+\beta}\right)} .
$$

Case $N \geqslant 4$ : From Proposition 1.9, we get

$$
\left\|T_{\partial_{j} b}^{\prime} u^{j}\right\|_{L_{T}^{1}\left(B_{2}^{N / 2-1+\beta}\right)} \lesssim\|\nabla b\|_{L_{T}^{2}\left(B_{\infty}^{-1}\right)}\|u\|_{L_{T}^{2}\left(B_{2}^{N / 2+\beta}\right)} .
$$

But replacing $\mathcal{Q} u$ by $b$ in the proof of (43), we also get

$$
\|b\|_{L_{T}^{2}\left(B_{\infty}^{0}\right)} \lesssim \varepsilon^{\alpha}\left(X_{\alpha}+\varepsilon^{-1 / 2} Y_{\alpha}\right)
$$

so that

$$
\left\|T_{\partial_{j} b}^{\prime} u^{j}\right\|_{L_{T}^{1}\left(B_{2}^{N / 2-1+\beta}\right)} \lesssim \varepsilon^{\alpha}\left(X_{\beta}+P_{\beta}\right)\left(X_{\alpha}+\varepsilon^{-1 / 2} Y_{\alpha}\right) .
$$

According to (41), the following inequality holds true

$$
\begin{aligned}
\left\|T_{\partial_{j} b}^{\prime} u^{j}\right\|_{L_{T}^{1}\left(B_{2}^{N / 2+\beta}\right)} & \lesssim\|\nabla b\|_{L_{T}^{\infty}\left(B_{2}^{N / 2-1}\right)}\|u\|_{L_{T}^{1}\left(B_{2}^{N / 2+1+\beta}\right)}, \\
& \lesssim \varepsilon^{\alpha-1}\|b\|_{L_{T}^{\infty}\left(\widetilde{B}_{\varepsilon \nu}^{N / 2+\alpha, \infty}\right)}\|u\|_{L_{T}^{1}\left(B_{2}^{N / 2-1+\beta}\right)} \\
& \lesssim \varepsilon^{\alpha-1} X_{\alpha}\left(X_{\beta}+P_{\beta}\right) .
\end{aligned}
$$

Using Proposition 1.4, (43) and (62), we infer that

$$
\begin{aligned}
\|b \operatorname{div} \mathcal{Q} u\|_{L_{T}^{1}\left(B_{2}^{N / 2+\beta-1}\right)} \lesssim & \|b\|_{L_{T}^{2}\left(B_{\infty}^{0}\right)}\|\operatorname{div} \mathcal{Q} u\|_{L_{T}^{2}\left(B_{2}^{N / 2-1+\beta}\right)} \\
& +\|\operatorname{div} \mathcal{Q} u\|_{L_{T}^{2}\left(B_{\infty}^{-1}\right)}\|b\|_{L_{T}^{2}\left(B_{2}^{N / 2+\beta}\right)}, \\
\lesssim & \varepsilon^{\alpha} X_{\beta}\left(X_{\alpha}+\varepsilon^{-1 / 2} Y_{\alpha}\right) .
\end{aligned}
$$

On the other hand, using (7) and (41), we gather that

$$
\begin{aligned}
\|b \operatorname{div} \mathcal{Q} u\|_{L_{T}^{1}\left(B_{2}^{N / 2}\right)} & \lesssim\|b\|_{L_{T}^{\infty}\left(B_{2}^{N / 2}\right)}\|\operatorname{div} \mathcal{Q} u\|_{L_{T}^{1}\left(B_{2}^{N / 2}\right)}, \\
& \lesssim \varepsilon^{\alpha-1} X_{\alpha} X_{0},
\end{aligned}
$$

$\|b \operatorname{div} \mathcal{Q} u\|_{L_{T}^{1}\left(B_{2}^{N / 2+\alpha}\right)}$

$$
\begin{aligned}
& \lesssim\|b\|_{L_{T}^{\infty}\left(B_{2}^{N / 2}\right)}\|\operatorname{div} \mathcal{Q} u\|_{L_{T}^{1}\left(B_{2}^{N / 2+\alpha}\right)}+\|b\|_{L_{T}^{2 / \alpha}\left(B_{2}^{N / 2+\alpha}\right)}\|\operatorname{div} \mathcal{Q} u\|_{L_{T}^{2 /(2-\alpha)}\left(B_{2}^{N / 2}\right)}, \\
& \lesssim \varepsilon^{\alpha-1}\left(\|b\|_{L_{T}^{\infty}\left(\widetilde{B}_{\varepsilon \nu}^{N / 2+\alpha, \infty}\right)}\|\mathcal{Q} u\|_{L_{T}^{1}\left(B_{2}^{N / 2+1+\alpha}\right)}\right.
\end{aligned}
$$


(67) $\lesssim \varepsilon^{\alpha-1} X_{\alpha}^{2}$.

$$
\left.+\|b\|_{L_{T}^{2 / \alpha}\left(\widetilde{B}_{\varepsilon \nu}^{N / 2+\alpha, 2 / \alpha}\right)}\|\mathcal{Q} u\|_{L_{T}^{2 /(2-\alpha)}\left(B_{2}^{N / 2+1}\right)}\right)
$$

Plugging inequalities (63)-(67) in (61), we conclude that for $\beta \in\{0, \alpha\}$,

$$
\|F\|_{L_{T}^{1}\left(\widetilde{B}_{\varepsilon \nu}^{N / 2+\beta, \infty}\right)} \lesssim \varepsilon^{\alpha}\left(X_{\beta}+P_{\beta}\right)\left(X_{\alpha}+\varepsilon^{-1 / 2} Y_{\alpha}\right) .
$$

Case $N=3$ : Estimates (64), (66) and (67) still hold in the case $N=3$ so that we only have to deal with (62), (63) and (65). According to Proposition 1.9, we have

$$
\begin{aligned}
\left\|T_{\partial_{j} b}^{\prime} u^{j}\right\|_{L_{T}^{1}\left(B_{2}^{1 / 2+\beta}\right)} & \lesssim\left\|\partial_{j} b\right\|_{L_{T}^{1 / \alpha}\left(B_{\infty}^{2 \alpha-2}\right)}\left\|u^{j}\right\|_{L_{T}^{1 /(1-\alpha)}\left(B_{2}^{5 / 2-2 \alpha+\beta}\right)}, \\
& \lesssim \varepsilon^{\alpha}\left(X_{\beta}+P_{\beta}\right)\left(\varepsilon^{-1 / \alpha} Y_{\alpha}^{1 / \alpha}\right) .
\end{aligned}
$$

Using inequality (44) and Proposition 1.4, we infer

$$
\begin{aligned}
\|b \operatorname{div} \mathcal{Q} u\|_{L_{T}^{1}\left(B_{2}^{1 / 2+\beta}\right)} \lesssim & \|b\|_{L_{T}^{1 / \alpha}\left(B_{\infty}^{2 \alpha-1}\right)}\|\operatorname{div} \mathcal{Q} u\|_{L_{T}^{1 /(1-\alpha)}\left(B_{2}^{3 / 2-2 \alpha+\beta}\right)} \\
& +\|\operatorname{div} \mathcal{Q} u\|_{L_{T}^{2}\left(B_{\infty}^{-1}\right)}\|b\|_{L_{T}^{2}\left(B_{2}^{3 / 2+\beta}\right)}, \\
\lesssim & \varepsilon^{\alpha} X_{\beta}\left(X_{\alpha}+\varepsilon^{-1 / \alpha} Y_{\alpha}^{1 / \alpha}+\varepsilon^{-2 \alpha /(1+2 \alpha)} Y_{\alpha}^{p_{\alpha}}\right) .
\end{aligned}
$$

Plugging inequalities (69), (64), (70), (66) and (67) in (61), we conclude that

$$
\|F\|_{L_{T}^{1}\left(\widetilde{B}_{\varepsilon \nu}^{3 / 2+\beta, \infty}\right)} \lesssim \varepsilon^{\alpha}\left(X_{\beta}+P_{\beta}\right)\left(X_{\alpha}+\varepsilon^{-1 / \alpha} Y_{\alpha}^{1 / \alpha}+\varepsilon^{-2 \alpha /(1+2 \alpha)} Y_{\alpha}^{p_{\alpha}}\right) .
$$

Case $N=2$ : Estimates (64), (66) and (67) also hold in the case $N=2$ so that we just have to concentrate on (62), (63) and (65). Let us notice that

$$
F=-T_{\operatorname{div} \mathcal{Q} u} b-\partial_{j} T_{b}^{\prime} u^{j} .
$$

According to Proposition 1.9, Proposition 1.3(v) and Hölder inequality, we have

$$
\begin{aligned}
& \left\|T_{\operatorname{div} \mathcal{Q} u} b\right\|_{L_{T}^{1}\left(B_{2}^{\beta}\right)} \\
& \lesssim\|\operatorname{div} \mathcal{Q} u\|_{L_{T}^{7 /(4-3 \alpha)}\left(B_{\infty}^{-1}\right)}\|b\|_{L_{T}^{7 /(3+3 \alpha)}\left(B_{2}^{1+\beta}\right)}, \\
& \lesssim \varepsilon^{(6 \alpha-1) / 7}\|\operatorname{div} \mathcal{Q} u\|_{L_{T}^{1}\left(B_{2}^{1+\alpha}\right)}^{(3-4 \alpha \alpha)}\|\operatorname{div} \mathcal{Q} u\|_{L_{T}^{4}\left(B_{\infty}^{\alpha-7 / 4}\right)}^{(4+4 \alpha) / 7}\|b\|_{L_{T}^{7 /(3+3 \alpha)}\left(\widetilde{B}_{\varepsilon \nu}^{1+\beta, 7 /(3+3 \alpha)}\right)}, \\
& \lesssim \varepsilon^{\alpha} X_{\beta}\left(X_{\alpha}+\varepsilon^{-1 / 4} Y_{\alpha}\right), \\
& \left\|\partial_{j} T_{b}^{\prime} u^{j}\right\|_{L_{T}^{1}\left(B_{2}^{\beta+\alpha-1 / 4}\right)} \lesssim\|b\|_{L_{T}^{4}\left(B_{\infty}^{\alpha-3 / 4}\right)}\|u\|_{L_{T}^{4 / 3}\left(B_{2}^{\beta+3 / 2}\right)}, \\
& \\
& \quad\left(X_{\beta}+P_{\beta}\right) Y_{\alpha} .
\end{aligned}
$$

On the other hand, we have by definition of hybrid Besov norms,

$$
\|F\|_{L_{T}^{1}\left(\widetilde{B}_{\varepsilon \nu}^{1+\beta, \infty)}\right)} \approx\left\|F_{B F}\right\|_{L_{T}^{1}\left(B_{2}^{\beta}\right)}+\varepsilon \nu\left\|F_{H F}\right\|_{L_{T}^{1}\left(B_{2}^{1+\beta}\right)}
$$

so that

$$
\begin{aligned}
\|F\|_{L_{T}^{1}\left(\widetilde{B}_{\varepsilon \nu}^{1+\beta, \infty}\right)} & \lesssim\left\|\left(T_{\operatorname{div} \mathcal{Q} u} b\right)_{B F}\right\|_{L_{T}^{1}\left(B_{2}^{\beta}\right)}+\left\|\left(\partial_{j} T_{b}^{\prime} u^{j}\right)_{B F}\right\|_{L_{T}^{1}\left(B_{2}^{\beta}\right)}+\varepsilon \nu\left\|F_{H F}\right\|_{L_{T}^{1}\left(B_{2}^{1+\beta}\right)}, \\
& \lesssim\left\|T_{\operatorname{div} \mathcal{Q} u} b\right\|_{L_{T}^{1}\left(B_{2}^{\beta}\right)}+\varepsilon^{\alpha-1 / 4}\left\|\partial_{j} T_{b}^{\prime} u^{j}\right\|_{L_{T}^{1}\left(B_{2}^{\beta+\alpha-1 / 4}\right)}+\varepsilon \nu\|F\|_{L_{T}^{1}\left(B_{2}^{1+\beta}\right)} .
\end{aligned}
$$


Making use of (72), (73), (64), (66) and (67), we eventually conclude that

$$
\|F\|_{L_{T}^{1}\left(\widetilde{B}_{\varepsilon \nu}^{1+\beta, \infty}\right)} \lesssim \varepsilon^{\alpha}\left(X_{\beta}+P_{\beta}\right)\left(X_{\alpha}+\varepsilon^{-1 / 4} Y_{\alpha}\right)
$$

\section{Estimates for $G$}

Case $N \geqslant 4$ : Using Proposition 1.4, Lemma 1.5 and inequality (62), we get

$$
\begin{aligned}
& \|K(\varepsilon b) \nabla b\|_{L_{T}^{1}\left(B_{2}^{N / 2-1+\beta}\right)} \\
& \quad \lesssim\|K(\varepsilon b)\|_{L_{T}^{2}\left(L^{\infty}\right)}\|\nabla b\|_{L_{T}^{2}\left(B_{2}^{N / 2-1+\beta}\right)}+\|\nabla b\|_{L_{T}^{2}\left(B_{\infty}^{-1}\right)}\|K(\varepsilon b)\|_{L_{T}^{2}\left(B_{2}^{N / 2+\beta}\right)}, \\
& \quad \lesssim\|\varepsilon b\|_{L_{T}^{2}\left(L^{\infty}\right)}\|\nabla b\|_{L_{T}^{2}\left(B_{2}^{N / 2-1+\beta}\right)}+\|\nabla b\|_{L_{T}^{2}\left(B_{\infty}^{-1}\right)}\|\varepsilon b\|_{L_{T}^{2}\left(B_{2}^{N / 2+\beta}\right)}, \\
& \quad \lesssim \varepsilon^{1+\alpha} X_{\beta}\left(X_{\alpha}+\varepsilon^{-1 / 2} Y_{\alpha}\right) .
\end{aligned}
$$

According to Proposition 1.9 and inequality (62),

$$
\begin{aligned}
\left\|T_{\nabla \Lambda^{-1} \operatorname{div} \mathcal{Q} u}^{\prime} u\right\|_{L_{T}^{1}\left(B_{2}^{N / 2-1+\beta}\right)} & \lesssim\left\|\nabla \Lambda^{-1} \operatorname{div} \mathcal{Q} u\right\|_{L_{T}^{2}\left(B_{\infty}^{-1}\right)}\|u\|_{L_{T}^{2}\left(B_{2}^{N / 2+\beta}\right)}, \\
& \lesssim \varepsilon^{\alpha}\left(X_{\alpha}+\varepsilon^{-1 / 2} Y_{\alpha}\right)\left(X_{\beta}+P_{\beta}\right) .
\end{aligned}
$$

Let us decompose the last two terms in $G$ in the following way:

$$
\begin{aligned}
u \cdot \nabla \Lambda^{-1} \operatorname{div} \mathcal{Q} u-\Lambda^{-1} \operatorname{div}(u \cdot \nabla u)= & \mathcal{Q} u \cdot \nabla \Lambda^{-1} \operatorname{div} \mathcal{Q} u-\Lambda^{-1}(\mathcal{Q} u \cdot \nabla \mathcal{Q} u) \\
& -\Lambda^{-1} \operatorname{div}(\mathcal{P} u \cdot \nabla \mathcal{P} u)-\Lambda^{-1} \operatorname{div}(\mathcal{Q} u \cdot \nabla \mathcal{P} u) \\
& -\Lambda^{-1} \operatorname{div}(\mathcal{P} u \cdot \nabla \mathcal{Q} u)+\mathcal{P} u \cdot \nabla \Lambda^{-1} \operatorname{div} \mathcal{Q} u
\end{aligned}
$$

Thanks to Proposition 1.4, we can easily bound the first two terms of the right-hand side:

$$
\begin{aligned}
\left\|\mathcal{Q} u \cdot \nabla \Lambda^{-1} \operatorname{div} \mathcal{Q} u-\Lambda^{-1}(\mathcal{Q} u \cdot \nabla \mathcal{Q} u)\right\|_{L_{T}^{1}\left(B_{2}^{N / 2-1+\beta}\right)} & \lesssim\|\mathcal{Q} u\|_{L_{T}^{2}\left(B_{\infty}^{0}\right)}\|\mathcal{Q} u\|_{L_{T}^{2}\left(B_{2}^{N / 2+\beta}\right)}, \\
& \lesssim \varepsilon^{\alpha} X_{\beta}\left(X_{\alpha}+\varepsilon^{-1 / 2} Y_{\alpha}\right) .
\end{aligned}
$$

For the third term, we just write

$$
\left\|\Lambda^{-1} \operatorname{div}(\mathcal{P} u \cdot \nabla \mathcal{P} u)\right\|_{L_{T}^{1}\left(B_{2}^{N / 2-1+\beta}\right)} \lesssim\|\mathcal{P} u\|_{L_{T}^{2}\left(B_{2}^{N / 2}\right)}\|\nabla \mathcal{P} u\|_{L_{T}^{2}\left(B_{2}^{N / 2-1+\beta}\right)} \lesssim P_{0} P_{\beta}
$$

Following the computations of step 2 (where $v$ has been replaced by $\mathcal{P} u$ ), we get

$$
\begin{aligned}
& \left\|\Lambda^{-1} \operatorname{div}(\mathcal{Q} u \cdot \nabla \mathcal{P} u)\right\|_{L_{T}^{1}\left(B_{2}^{N / 2-1+\beta}\right)} \lesssim \varepsilon^{4 \alpha /(2+N+2 \beta)}\left(P_{0} X_{\beta}+P_{\beta}\left(X_{\alpha}+\varepsilon^{-1 / 2} Y_{\alpha}\right)\right) \\
& \left\|\Lambda^{-1} \operatorname{div}(\mathcal{P} u \cdot \nabla \mathcal{Q} u)\right\|_{L_{T}^{1}\left(B_{2}^{N / 2-1+\beta}\right)} \lesssim \varepsilon^{2 \alpha /(2+N+2 \beta)}\left(P_{0} X_{\beta}+P_{\beta}\left(X_{\alpha}+\varepsilon^{-1 / 2} Y_{\alpha}\right)\right) \\
& \left\|\mathcal{P} u \cdot \nabla \Lambda^{-1} \operatorname{div} \mathcal{Q} u\right\|_{L_{T}^{1}\left(B_{2}^{N / 2-1+\beta}\right)} \lesssim \varepsilon^{2 \alpha /(2+N+2 \beta)}\left(P_{0} X_{\beta}+P_{\beta}\left(X_{\alpha}+\varepsilon^{-1 / 2} Y_{\alpha}\right)\right)
\end{aligned}
$$

Thanks to (42) and (75)-(81), we end up with

$$
\begin{aligned}
\|G\|_{L_{T}^{1}\left(B_{2}^{N / 2-1+\beta}\right) \lesssim} & P_{0} P_{\beta}+\varepsilon^{\alpha} X_{\beta}\left(X_{\alpha}+\varepsilon^{-1 / 2} Y_{\alpha}\right) \\
& +\varepsilon^{2 \alpha /(2+N+2 \beta)}\left(P_{0} X_{\beta}+P_{\beta}\left(X_{\alpha}+\varepsilon^{-1 / 2} Y_{\alpha}\right)\right) .
\end{aligned}
$$


Case $N=3$ : According to Proposition 1.4 and Lemma 1.5, we have

$$
\begin{aligned}
\|K(\varepsilon b) \nabla b\|_{B_{2}^{1 / 2+\beta}} & \lesssim\|K(\varepsilon b)\|_{L^{\infty}}\|\nabla b\|_{B_{2}^{1 / 2+\beta}}+\|\nabla b\|_{B_{\infty}^{-1}}\|K(\varepsilon b)\|_{B_{2}^{3 / 2+\beta}}, \\
& \lesssim\|\varepsilon b\|_{L^{\infty}}\|b\|_{B_{2}^{3 / 2+\beta}}+\|b\|_{B_{\infty}^{0}}\|\varepsilon b\|_{B_{2}^{3 / 2+\beta}}, \\
& \lesssim \varepsilon\|b\|_{B_{2}^{3 / 2+\beta}}\left(\left\|b_{B F}\right\|_{B_{\infty}^{0}}+\left\|b_{H F}\right\|_{B_{\infty}^{0}}\right)
\end{aligned}
$$

where the notations $b_{B F}$ and $b_{H F}$ have been defined in Section 1 . Hence,

$$
\begin{aligned}
& \left\|\frac{K(\varepsilon b) \nabla b}{\varepsilon}\right\|_{L_{T}^{1}\left(B_{2}^{1 / 2+\beta}\right)} \\
& \quad \lesssim\left\|b_{B F}\right\|_{L_{T}^{2}\left(B_{\infty}^{0}\right)}\|b\|_{L_{T}^{2}\left(B_{2}^{3 / 2+\beta}\right)}+\left\|b_{H F}\right\|_{L_{T}^{1 /(1-\alpha)}\left(B_{\infty}^{0}\right)}\|b\|_{L_{T}^{1 / \alpha}\left(B_{2}^{3 / 2+\beta}\right)} .
\end{aligned}
$$

Let us remark now that we can replace $\mathcal{Q} u$ with $b_{B F}$ in the proof of (44). This yields

$$
\left\|b_{B F}\right\|_{L_{T}^{2}\left(B_{\infty}^{0}\right)} \lesssim \varepsilon^{\alpha}\left(X_{\alpha}+\varepsilon^{-2 \alpha /(1+2 \alpha)} Y_{\alpha}^{p_{\alpha}}\right) .
$$

On the other hand, according to (7), we have

$$
\|b\|_{L_{T}^{1 / \alpha}\left(B_{2}^{3 / 2+\beta}\right)} \lesssim \varepsilon^{2 \alpha-1}\|b\|_{L_{T}^{1 / \alpha}\left(\widetilde{B}_{\varepsilon \nu}^{3 / 2+\beta, 1 / \alpha}\right)},
$$

and according to Proposition 1.3(iii) and (v), and (8),

$$
\begin{aligned}
\left\|b_{H F}\right\|_{L_{T}^{1 /(1-\alpha)}\left(B_{\infty}^{0}\right)} & \lesssim\left\|b_{H F}\right\|_{L_{T}^{1}\left(B_{2}^{3 / 2+\alpha}\right)}^{(1-2 \alpha) /(1-\alpha)}\left\|b_{H F}\right\|_{L_{T}^{1 / \alpha}\left(B_{\infty}^{-1+2 \alpha}\right)}^{\alpha /(1-\alpha)}, \\
& \lesssim\left(\varepsilon \nu\left\|b_{H F}\right\|_{L_{T}^{1}\left(\widetilde{B}_{\varepsilon \nu}^{3 / 2+\alpha, 1}\right)}\right)^{(1-2 \alpha) /(1-\alpha)} \varepsilon^{\alpha^{2} /(1-\alpha)}\left(\varepsilon^{-\alpha} Y_{\alpha}^{1 / \alpha}\right)^{\alpha /(1-\alpha)}, \\
& \lesssim \varepsilon^{1-\alpha}\left(X_{\alpha}+\varepsilon^{-\alpha} Y_{\alpha}^{1 / \alpha}\right) .
\end{aligned}
$$

Plugging this latter inequality, (84) and (85) in (83), we conclude that

$$
\left\|\frac{K(\varepsilon b) \nabla b}{\varepsilon}\right\|_{L_{T}^{1}\left(B_{2}^{1 / 2+\beta}\right)} \lesssim \varepsilon^{\alpha} X_{\beta}\left(X_{\alpha}+\varepsilon^{-2 \alpha /(1+2 \alpha)} Y_{\alpha}^{p_{\alpha}}+\varepsilon^{-\alpha} Y_{\alpha}^{1 / \alpha}\right) .
$$

The other terms can be treated by following the proof in the case $N \geqslant 4$. One just has to use (44) instead of (43) which amounts to replace $\varepsilon^{-1 / 2} Y_{\alpha}$ with $\varepsilon^{-2 \alpha /(1+2 \alpha)} Y_{\alpha}^{p_{\alpha}}$. Finally we conclude that

$$
\begin{aligned}
\|G\|_{L_{T}^{1}\left(B_{2}^{N / 2-1+\beta}\right) \lesssim} & P_{0} P_{\beta}+\varepsilon^{\alpha} X_{\beta}\left(X_{\alpha}+\varepsilon^{-2 \alpha /(1+2 \alpha)} Y_{\alpha}^{p_{\alpha}}+\varepsilon^{-\alpha} Y_{\alpha}^{1 / \alpha}\right) \\
& +\varepsilon^{2 \alpha /(5+2 \beta)}\left(P_{0} X_{\beta}+P_{\beta}\left(X_{\alpha}+\varepsilon^{-2 \alpha /(1+2 \alpha)} Y_{\alpha}^{p_{\alpha}}\right)\right) .
\end{aligned}
$$

Case $N=2$ : Bony decomposition for $K(\varepsilon b) \nabla b$ yields

$$
K(\varepsilon b) \nabla b=T_{\nabla b} K(\varepsilon b)+R(\nabla b, K(\varepsilon b))+T_{K(\varepsilon b)} \nabla b .
$$

According to Proposition 1.9 and Lemma 1.5, we have

$$
\begin{aligned}
\left\|T_{\nabla b} K(\varepsilon b)\right\|_{B_{2}^{\beta}} & \lesssim\|\nabla b\|_{B_{\infty}^{-1}}\|K(\varepsilon b)\|_{B_{2}^{1+\beta}} \lesssim \varepsilon\|b\|_{B_{\infty}^{0}}\|b\|_{B_{2}^{1+\beta}}, \\
\|R(\nabla b, K(\varepsilon b))\|_{B_{2}^{\beta}} & \lesssim\|\nabla b\|_{B_{2}^{\beta}}\|K(\varepsilon b)\|_{B_{14 /(3-4 \alpha)}^{(3-4 \alpha) / 7}} \lesssim \varepsilon\|b\|_{B_{2}^{1+\beta}\|b\|_{B_{14 /(3-4 \alpha)}^{(3-4 \alpha) / 7}}} \\
\left\|T_{K(\varepsilon b)} \nabla b\right\|_{B_{2}^{\beta}} & \lesssim\|K(\varepsilon b)\|_{L^{\infty}}\|\nabla b\|_{B_{2}^{\beta}} \lesssim \varepsilon\|b\|_{L^{\infty}}\|b\|_{B_{2}^{1+\beta}}
\end{aligned}
$$


Hence, using the chain of embeddings $B_{6 /(3-4 \alpha)}^{(3-4 \alpha) / 3} \hookrightarrow B_{14 /(3-4 \alpha)}^{(3-4 \alpha) / 7} \hookrightarrow B_{\infty}^{0} \hookrightarrow L^{\infty}$,

$$
\begin{aligned}
\|K(\varepsilon b) \nabla b\|_{B_{2}^{\beta}} & \lesssim \varepsilon\|b\|_{B_{2}^{1+\beta}}\|b\|_{B_{14 /(3-4 \alpha)}^{(3-4 \alpha) / 7}}, \\
& \lesssim \varepsilon\|b\|_{B_{2}^{1+\beta}}\left(\left\|b_{B F}\right\|_{B_{14 /(3-4 \alpha)}^{(3-4 \alpha) / 7}}+\left\|b_{H F}\right\|_{B_{6 /(3-4 \alpha)}^{(3-4 \alpha) / 3}}\right) .
\end{aligned}
$$

Therefore, according to Hölder inequality, Proposition 1.3(v) and (8),

$$
\begin{aligned}
\left\|\frac{K(\varepsilon b) \nabla b}{\varepsilon}\right\|_{L_{T}^{1}\left(B_{2}^{\beta}\right)} \lesssim & \|b\|_{L_{T}^{7 /(3+3 \alpha)}\left(B_{2}^{1+\beta}\right)}\left\|b_{B F}\right\|_{L_{T}^{7 /(4-3 \alpha)}\left(B_{14 /(3-4 \alpha)}^{(3-4 \alpha) / 7}\right)} \\
& +\|b\|_{L_{T}^{1 / \alpha}\left(B_{2}^{1+\beta}\right)}\left\|b_{H F}\right\|_{L_{T}^{1 /(1-\alpha)}\left(B_{6 /(3-4 \alpha)}^{(3-4 \alpha) / 3}\right)} \\
\lesssim & \varepsilon^{(6 \alpha-1) / 7}\|b\|_{L_{T}^{7 /(3+3 \alpha)}\left(\widetilde{B}_{\varepsilon \nu}^{1+\beta, 7 /(3+3 \alpha)}\right)}\left\|b_{B F}\right\|_{L_{T}^{1}\left(B_{2}^{2+\alpha}\right)}^{(3-4 \alpha) / 7}\left\|b_{B F}\right\|_{L_{T}^{4}\left(B_{\infty}^{\alpha-3 / 4}\right)}^{(4+4 \alpha) / 7} \\
& +\varepsilon^{2 \alpha-1}\|b\|_{L_{T}^{1 / \alpha}\left(\widetilde{B}_{\varepsilon \nu}^{1+\beta, 1 / \alpha}\right)}\left\|b_{H F}\right\|_{L_{T}^{1}\left(B_{2}^{1+\alpha}\right)}^{1-4 \alpha / 3}\left\|b_{H F}\right\|_{L_{T}^{4}\left(B_{\infty}^{\alpha-3 / 4}\right)}^{4 \alpha / 3} \\
\lesssim & \varepsilon^{\alpha} X_{\beta}\|b\|_{L_{T}^{1}\left(\widetilde{B}_{\varepsilon \nu}^{1+\alpha, 1}\right)}^{(3-4 \alpha) / 7}\left(\varepsilon^{-1 / 4}\|b\|_{L_{T}^{4}\left(B_{\infty}^{\alpha-3 / 4}\right)}\right)^{(4+4 \alpha) / 7} \\
& +\varepsilon^{\alpha} X_{\beta}\|b\|_{L_{T}^{1}\left(\widetilde{B}_{\varepsilon \nu}^{1+\alpha, 1}\right)}^{1-4 \alpha / 3}\left(\varepsilon^{-1 / 4}\left\|b_{H F}\right\|_{L_{T}^{4}\left(B_{\infty}^{\alpha-3 / 4}\right)}\right)^{4 \alpha / 3} .
\end{aligned}
$$

We eventually find

$$
\left\|\frac{K(\varepsilon b) \nabla b}{\varepsilon}\right\|_{L_{T}^{1}\left(B_{2}^{\beta}\right)} \lesssim \varepsilon^{\alpha} X_{\beta}\left(X_{\alpha}+\varepsilon^{-1 / 4} Y_{\alpha}\right) .
$$

The other terms in $G$ have been treated in step 2. One just has to replace $v$ with $\mathcal{P} u$ or $\mathcal{Q} u$, whichever is appropriate. We eventually get

$$
\begin{aligned}
\|G\|_{L_{T}^{1}\left(B_{2}^{N / 2-1+\beta}\right) \lesssim} & P_{0} P_{\beta}+\varepsilon^{\alpha} X_{\beta}\left(X_{\alpha}+\varepsilon^{-1 / 4} Y_{\alpha}\right) \\
& +\varepsilon^{\alpha /(2+\beta)}\left(P_{0} X_{\beta}+P_{\beta}\left(X_{\alpha}+\varepsilon^{-1 / 4} Y_{\alpha}\right)\right) .
\end{aligned}
$$

Let us now choose the values of $p$ and $r$ in $V_{\nu, \varepsilon}^{p, r}$. A possible choice is $p=1 / \alpha$ and $r=2 /(2-\alpha)$. Indeed, using an interpolation, we have, if $N \geqslant 4$,

$$
\begin{aligned}
\|\nabla u\|_{L_{T}^{1 / \alpha}\left(B_{\infty}^{2 \alpha-2}\right)} & \lesssim\|\nabla \mathcal{Q} u\|_{L_{T}^{2}\left(B_{\infty}^{\alpha-3 / 2}\right)}^{2 \alpha}\|\nabla \mathcal{Q} u\|_{L_{T}^{\infty}\left(B_{\infty}^{\alpha-2}\right)}^{1-2 \alpha}+\|\nabla \mathcal{P} u\|_{L_{T}^{1 / \alpha}\left(B_{2}^{N / 2+2 \alpha-2}\right)} \\
& \lesssim \varepsilon^{\alpha}\left(X_{\alpha}+\varepsilon^{-1 / 2} Y_{\alpha}\right)+P_{0}
\end{aligned}
$$

if $N=3$,

$$
\begin{aligned}
\|\nabla u\|_{L_{T}^{1 / \alpha}\left(B_{\infty}^{2 \alpha-2}\right)} & \lesssim\|\nabla \mathcal{Q} u\|_{L_{T}^{1 / \alpha}\left(B_{\infty}^{2 \alpha-2}\right)}+\|\nabla \mathcal{P} u\|_{L_{T}^{1 / \alpha}\left(B_{2}^{2 \alpha-1 / 2}\right)}, \\
& \lesssim \varepsilon^{\alpha}\left(\varepsilon^{-\alpha} Y_{\alpha}^{1 / \alpha}\right)+P_{0}
\end{aligned}
$$

and if $N=2$,

$$
\begin{aligned}
\|\nabla u\|_{L_{T}^{1 / \alpha}\left(B_{\infty}^{2 \alpha-2}\right)} & \lesssim\|\nabla \mathcal{Q} u\|_{L_{T}^{4}\left(B_{\infty}^{\alpha-7 / 4}\right)}^{4 \alpha}\|\nabla \mathcal{Q} u\|_{L_{T}^{\infty}\left(B_{\infty}^{\alpha-2}\right)}^{1-4 \alpha}+\|\nabla \mathcal{P} u\|_{L_{T}^{1 / \alpha}\left(B_{2}^{2 \alpha-1}\right)}, \\
& \lesssim \varepsilon^{\alpha}\left(X_{\alpha}+\varepsilon^{-1 / 4} Y_{\alpha}\right)+P_{0} .
\end{aligned}
$$

On the other hand, for any $N \geqslant 2$,

$$
\begin{aligned}
\|\nabla u\|_{L_{T}^{2 /(2-\alpha)}\left(L^{\infty}\right)} & \lesssim\|u\|_{L_{T}^{2 /(2-\alpha)}\left(B_{2}^{N / 2+1}\right)} \\
& \lesssim P_{\alpha}+X_{\alpha}
\end{aligned}
$$


According to (60), we thus have

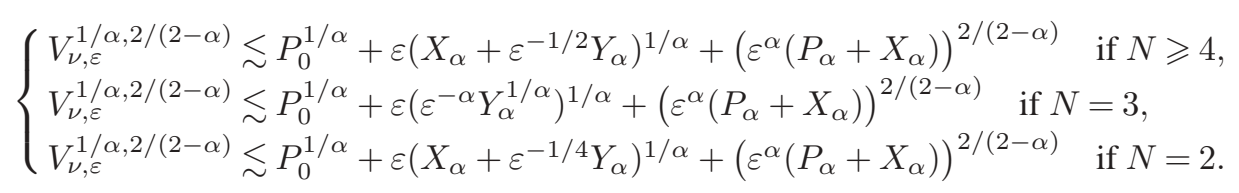

Plugging this latter inequality, (68) and (82) in (59), we eventually get, if $N \geqslant 4$,

$$
\begin{aligned}
X_{\beta} \leqslant & C \mathrm{e}^{C\left(P_{0}^{1 / \alpha}+\varepsilon\left(X_{\alpha}+\varepsilon^{-1 / 2} Y_{\alpha}\right)^{1 / \alpha}+\left(\varepsilon^{\alpha}\left(P_{\alpha}+X_{\alpha}\right)\right)^{2 /(2-\alpha)}\right)} \\
& \times\left(X_{\beta}^{0}+P_{0} P_{\beta}+\varepsilon^{\alpha_{N}}\left(P_{0} X_{\beta}+\left(X_{\beta}+P_{\beta}\right)\left(X_{\alpha}+\varepsilon^{-1 / 2} Y_{\alpha}\right)\right)\right) .
\end{aligned}
$$

If $N=3$, we use (71) and (87) and get

$$
\begin{aligned}
X_{\beta} \leqslant & C \mathrm{e}^{C\left(P_{0}^{1 / \alpha}+\varepsilon\left(\varepsilon^{-\alpha} Y_{\alpha}^{1 / \alpha}\right)^{1 / \alpha}+\left(\varepsilon^{\alpha}\left(P_{\alpha}+X_{\alpha}\right)\right)^{2 /(2-\alpha)}\right)} \\
& \times\left(X_{\beta}^{0}+P_{0} P_{\beta}+\varepsilon^{\alpha_{3}}\left(P_{0} X_{\beta}+\left(X_{\beta}+P_{\beta}\right)\left(X_{\alpha}+\varepsilon^{-\alpha} Y_{\alpha}^{1 / \alpha}+\varepsilon^{-2 \alpha /(1+2 \alpha)} Y_{\alpha}^{p_{\alpha}}\right)\right)\right) .
\end{aligned}
$$

If $N=2$, we plug (90), (74) and (89) in (59) and get

$$
\begin{aligned}
X_{\beta} \leqslant & C \mathrm{e}^{C\left(P_{0}^{1 / \alpha}+\varepsilon\left(X_{\alpha}+\varepsilon^{-1 / 4} Y_{\alpha}\right)^{1 / \alpha}+\left(\varepsilon^{\alpha}\left(P_{\alpha}+X_{\alpha}\right)\right)^{2 /(2-\alpha)}\right)} \\
& \times\left(X_{\beta}^{0}+P_{0} P_{\beta}+\varepsilon^{\alpha_{2}}\left(P_{0} X_{\beta}+\left(X_{\beta}+P_{\beta}\right)\left(X_{\alpha}+\varepsilon^{-1 / 4} Y_{\alpha}\right)\right)\right) .
\end{aligned}
$$

In the inequalities above, we set $\alpha_{N} \stackrel{\text { def }}{=} 2 \alpha /(2+N+2 \alpha)$.

\section{Fourth step: bootstrap}

From now on, the proof is the same in any dimension $N \geqslant 2$. Denote $X \stackrel{\text { def }}{=} X_{0}+X_{\alpha}$, $V \stackrel{\text { def }}{=} V_{0}+V_{\alpha}, W \stackrel{\text { def }}{=} W_{0}+W_{\alpha}$ and $X^{0} \stackrel{\text { def }}{=} X_{0}^{0}+X_{\alpha}^{0}$. With this new notation, putting together estimates (38), (49) or (50) or (58), and (91) or (92) or (93), we gather

$$
W \leqslant C \mathrm{e}^{C(V+X)}\left(\varepsilon^{\alpha_{N}}\left(X^{2}+V\left(X^{0}+X+X^{2}+V^{2}\right)\right)+W^{2}\left(1+\varepsilon^{\alpha_{N}} V\right)\right),
$$

$$
\begin{aligned}
X \leqslant & C \mathrm{e}^{C\left(\varepsilon\left(X+X^{2}\right)^{1 / \alpha}+\left(\varepsilon^{\alpha} X\right)^{2 /(2-\alpha)}\right)} \mathrm{e}^{C\left((V+W)^{1 / \alpha}+\varepsilon\left(X^{0}+(V+W)^{2}\right)^{1 / \alpha}+\varepsilon^{2 \alpha /(2-\alpha)}(V+W)^{2 /(2-\alpha)}\right)} \\
& \times\left(X^{0}+(V+W)\left(V+W+\varepsilon^{\alpha_{N}}\left(X^{0}+V^{2}+W^{2}\right)\right)\right. \\
& \left.\quad+\varepsilon^{\alpha_{N}} X\left(X^{0}+V+W+X+X^{2}\right)\right) .
\end{aligned}
$$

A bootstrap argument will enable us to get a bound for $(b, u)$ from the two estimates above. More precisely, we shall prove the following lemma:

LemmA 5.2. - Suppose that $v \in F_{T_{0}}^{N / 2} \cap F_{T_{0}}^{N / 2+\alpha}$ for a finite or infinite $T_{0}$. Then there exists an $\varepsilon_{0}>0$ depending only on $\alpha, N, V\left(T_{0}\right)$ and on the norm of the compressible data $\left(b_{0}, \mathcal{Q} u_{0}, \mathcal{Q} f\right)$ in

$$
B^{N / 2-1} \cap B^{N / 2+\alpha} \times\left(B^{N / 2-1} \cap B^{N / 2-1+\alpha}\right)^{N} \times L^{1}\left(\mathbb{R}^{+} ;\left(B^{N / 2-1} \cap B^{N / 2-1+\alpha}\right)^{N}\right)
$$

and such that if $\varepsilon \leqslant \varepsilon_{0}$ and $(b, u) \in E_{\varepsilon \nu, T}^{N / 2} \cap E_{\varepsilon \nu, T}^{N / 2+\alpha}$ and $\varepsilon|b| \leqslant 3 / 4$ for $a T \leqslant T_{0}$, then the following estimates hold with the constant $C=C(N, \mu, \lambda, P, \alpha)$ appearing in (94) and (95)

$$
X(T) \leqslant X_{M} \stackrel{\text { def }}{=} 16 C \mathrm{e}^{C V^{1 / \alpha}\left(T_{0}\right)}\left(X^{0}+V^{2}\left(T_{0}\right)\right),
$$


$W(T) \leqslant \varepsilon^{\alpha_{N}} W_{M} \stackrel{\text { def }}{=} 4 \varepsilon^{\alpha_{N}} C \mathrm{e}^{C\left(V\left(T_{0}\right)+X_{M}\right)}\left(X_{M}^{2}+V\left(T_{0}\right)\left(X^{0}+X_{M}+X_{M}^{2}+V^{2}\left(T_{0}\right)\right)\right)$.

Proof. - Let $I \stackrel{\text { def }}{=}\left\{t \leqslant T \mid X(t) \leqslant X_{M}\right.$ and $\left.W(t) \leqslant \varepsilon^{\alpha_{N}} W_{M}\right\}$.

Obviously, $X$ and $W$ are continuous non-decreasing functions so that $I$ is a closed interval of $\mathbb{R}^{+}$with lower bound 0 . If, say, $C \geqslant 1$, then $I$ is not empty. With no loss of generality, one can assume that $I=\left[0, T^{\star}\right]$ for a finite positive $T^{\star}$. Choose $\varepsilon$ small enough so that the following conditions are fulfilled:

$$
\left\{\begin{array}{l}
C \mathrm{e}^{C\left(V\left(T_{0}\right)+X_{M}\right)} \varepsilon^{\alpha_{N}} W_{M}\left(1+\varepsilon^{\alpha_{N}} V\left(T_{0}\right)\right) \leqslant 1 / 2 \\
\mathrm{e}^{C\left(\varepsilon\left(X_{M}+X_{M}^{2}\right)^{1 / \alpha}+\left(\varepsilon^{\alpha} X_{M}\right)^{2 /(2-\alpha)}\right) \leqslant 2} \\
\mathrm{e}^{C\left(\left(V\left(T_{0}\right)+\varepsilon^{\alpha} W_{M}\right)^{1 / \alpha}+\varepsilon\left(X^{0}+\left(V\left(T_{0}\right)+\varepsilon^{\alpha_{N}} W_{M}\right)^{2}\right)^{1 / \alpha}+\varepsilon^{2 \alpha /(2-\alpha)}\left(V\left(T_{0}\right)+\varepsilon^{\alpha_{N}} W_{M}\right)^{2 /(2-\alpha)}\right)} \\
\quad \leqslant 2 \mathrm{e}^{C V^{1 / \alpha}\left(T_{0}\right)} \\
X^{0}+\left(V\left(T_{0}\right)+\varepsilon^{\alpha_{N}} W_{M}\right)\left(V\left(T_{0}\right)+\varepsilon^{\alpha_{N}} W_{M}+\varepsilon^{\alpha_{N}}\left(X^{0}+V^{2}\left(T_{0}\right)+\varepsilon^{2 \alpha_{N}} W_{M}^{2}\right)\right) \\
\quad \leqslant 2\left(X^{0}+V^{2}\left(T_{0}\right)\right) \\
\quad \mathrm{e}^{C V^{1 / \alpha}\left(T_{0}\right)} \varepsilon^{\alpha_{N}}\left(X^{0}+V\left(T_{0}\right)+\varepsilon^{\alpha_{N}} W_{M}+X_{M}+X_{M}^{2}\right) \leqslant 1 / 12
\end{array}\right.
$$

From inequalities (94) and (95), we conclude that

$$
\begin{aligned}
X\left(T^{\star}\right) & \leqslant 12 C \mathrm{e}^{C V^{1 / \alpha}\left(T_{0}\right)}\left(X^{0}+V^{2}(T)\right), \\
W\left(T^{\star}\right) & \leqslant 2 \varepsilon^{\alpha_{N}} C \mathrm{e}^{C\left(V\left(T_{0}\right)+X_{M}\right)}\left(X_{M}^{2}+V\left(T_{0}\right)\left(X^{0}+X_{M}+X_{M}^{2}+V^{2}\left(T_{0}\right)\right)\right) .
\end{aligned}
$$

This means that $T^{\star}=T$.

\section{Last step: continuation argument}

First of all, we have to state the existence of a local solution in $E_{\varepsilon \nu, T}^{N / 2} \cap E_{\varepsilon \nu, T}^{N / 2+\alpha}$ :

Proposition 5.3. - Suppose that $N \geqslant 2,0<\alpha<1, \quad b_{0} \in B^{N / 2-1} \cap B^{N / 2+\alpha}$, $u_{0} \in B^{N / 2-1} \cap B^{N / 2-1+\alpha}$ and $f \in L^{1}\left(\mathbb{R}^{+} ; B^{N / 2-1} \cap B^{N / 2-1+\alpha}\right)$. Then for all $\varepsilon<\left\|b_{0}\right\|_{L^{\infty}}^{-1}$ there exists a time $T$ such that (1) has a unique solution $(b, u) \in E_{\varepsilon \nu, T}^{N / 2} \cap E_{\varepsilon \nu, T}^{N / 2+\alpha}$ with $\varepsilon|b|<1$. Moreover, the time $T$ may be bounded by below by

$$
\min \left(\eta, \max \left\{t>0, \sum_{q \in \mathbb{Z}} 2^{q(N / 2-1+\alpha)}\left(\frac{1-\mathrm{e}^{-c t 2^{2 q}}}{c}\right)\left(\left\|\Delta_{q} u_{0}\right\|_{L^{2}}+\left\|\Delta_{q} f\right\|_{L^{\infty}\left(L^{2}\right)}\right) \leqslant \eta\right\}\right)
$$

where $c=c(\lambda, \mu)$ and $\eta$ is a non-increasing positive function of $\varepsilon\left\|b_{0}\right\|_{L^{\infty}},\left\|b_{0}\right\|_{B_{2}^{N / 2+\alpha}}$, $\left\|u_{0}\right\|_{B_{2}^{N / 2-1+\alpha}},\|f\|_{L_{T}^{1}\left(B_{2}^{N / 2-1+\alpha}\right)}$ and depends also on $\lambda, \mu, \varepsilon, P, \alpha$ and $N$.

Proof. - Making the change of function $\rho_{0}=1+\varepsilon b_{0}$ and $\rho=1+\varepsilon b$, and using that

$$
\begin{aligned}
& \rho_{0}-1 \in B^{N / 2} \cap B^{N / 2+\alpha}, \quad u_{0} \in B^{N / 2-1+\alpha}, \quad f \in L^{1}\left(\mathbb{R}^{+} ; B^{N / 2-1+\alpha}\right) \\
& \text { and } \inf _{x \in \mathbb{R}^{N}} \rho_{0}(x)>0
\end{aligned}
$$

one can apply Theorem 4.2 in [7]. From it, we get the existence of a finite time $T$ bounded from below as required and such that (1) has a unique solution $(b, u)$ with

$$
\begin{aligned}
& b \in C\left([0, T] ; B^{N / 2} \cap B^{N / 2+\alpha}\right), \quad \varepsilon|b|<1 \\
& \quad \text { and } u \in L^{1}\left(0, T ; B^{N / 2+1+\alpha}\right) \cap C\left([0, T] ; B^{N / 2-1+\alpha}\right) .
\end{aligned}
$$


Since $T$ is finite, proving that furthermore $b \in C\left([0, T] ; B^{N / 2-1}\right)$ and $u \in C\left([0, T] ; B^{N / 2-1}\right)$ will entail that $(b, u) \in E_{\varepsilon \nu, T}^{N / 2} \cap E_{\varepsilon \nu, T}^{N / 2+\alpha}$. From the first equation of (1), we readily get that $\partial_{t} b \in L^{2 /(1-\alpha)}\left(0, T ; B^{N / 2-1}\right)$. Indeed, by interpolation, $u \in L^{2 /(1-\alpha)}\left(0, T ; B^{N / 2}\right)$. Since $b_{0} \in B^{N / 2-1}$, we conclude that $b \in C^{(1+\alpha) / 2}\left([0, T] ; B^{N / 2-1}\right)$. Similarly, staring at the momentum equation in (1), one gets $\partial_{t} u-f \in L^{2 /(2-\alpha)}\left(0, T ; B^{N / 2-1}\right)$. Denoting by $u_{L}$ the solution to

$$
\partial_{t} u_{L}-\mathcal{A} u_{L}=f, \quad u_{L}(0)=u_{0},
$$

we conclude that $\left(u-u_{L}\right) \in C^{\alpha}\left([0, T] ; B^{N / 2-1}\right)$. Moreover, from Proposition 7.3, we have $u_{L} \in C\left([0, T] ; B^{N / 2-1}\right)$.

Now, suppose that for a $\left.\left.T_{0} \in\right] 0,+\infty\right]$, we have $v \in F_{T_{0}}^{N / 2} \cap F_{T_{0}}^{N / 2+\alpha}$ and let us turn to the proof that the life span $T_{\varepsilon}$ of the solution to (1) satisfies $T_{\varepsilon} \geqslant T_{0}$ if $\varepsilon$ is small enough.

More precisely, denote

$$
T_{\varepsilon} \stackrel{\text { def }}{=} \sup \left\{T \in \mathbb{R}^{+} \mid(b, u) \in E_{\varepsilon \nu, T}^{N / 2} \cap E_{\varepsilon \nu, T}^{N / 2+\alpha} \text { and } \forall t \in[0, T], \forall x \in \mathbb{R}^{N},|\varepsilon b(t, x)| \leqslant 3 / 4\right\} .
$$

According to Proposition 5.3, the time $T_{\varepsilon}$ is well defined and positive as soon as $\left|\varepsilon b_{0}\right|<3 / 4$.

Let us assume that $T_{\varepsilon}$ is finite and satisfies $T_{\varepsilon} \leqslant T_{0}$. According to Lemma 5.2, we have, for any $T<T_{\varepsilon}$ and $\varepsilon \leqslant \varepsilon_{0}$,

$$
X(T) \leqslant X_{M} \quad \text { and } \quad W(T) \leqslant \varepsilon^{\alpha_{N}} W_{M} .
$$

From the first inequality and (41), we gather that

$$
\varepsilon\|b\|_{L_{T}^{\infty}\left(B_{2}^{N / 2}\right)} \leqslant \varepsilon^{\alpha} \nu^{\alpha-1} X_{M} .
$$

Obviously, changing once more $\varepsilon_{0}$ if necessary, this entails

$$
\forall x \in \mathbb{R}^{N}, \quad \forall 0 \leqslant t<T_{\varepsilon}, \quad \varepsilon|b(t, x)| \leqslant 1 / 2 .
$$

We are now going to prove that $(b, u)$ can be continued in $T_{\varepsilon}$ as a solution of (1) belonging to $E_{\varepsilon \nu, T_{\varepsilon}+\tau}^{N / 2} \cap E_{\varepsilon \nu, T_{\varepsilon}+\tau}^{N / 2+\alpha}$ for a small $\tau>0$. According to (96), we have

$$
\begin{gathered}
b \in L^{1}\left(0, T_{\varepsilon} ; \widetilde{B}_{\varepsilon \nu}^{N / 2,1} \cap \widetilde{B}_{\varepsilon \nu}^{N / 2+\alpha, 1}\right) \cap C_{b}\left(\left[0, T_{\varepsilon}\left[; \widetilde{B}_{\varepsilon \nu}^{N / 2, \infty} \cap \widetilde{B}_{\varepsilon \nu}^{N / 2+\alpha, \infty}\right),\right.\right. \\
u \in L^{1}\left(0, T_{\varepsilon} ; B_{2}^{N / 2+1} \cap B_{2}^{N / 2+\alpha+1}\right) \cap C_{b}\left(\left[0, T_{\varepsilon}\left[; B_{2}^{N / 2-1} \cap B_{2}^{N / 2-1+\alpha}\right),\right.\right.
\end{gathered}
$$

so that, using Proposition 1.4 , this is not hard to get $\partial_{t} u-\mathcal{A} u \in L^{1}\left(0, T_{\varepsilon} ; B^{N / 2-1+\alpha}\right)$. According to Proposition 7.3, this entails

$$
\sum_{q \in \mathbb{Z}} 2^{q(N / 2-1+\alpha)}\left\|\Delta_{q} u\right\|_{L_{T_{\varepsilon}}^{\infty}\left(L^{2}\right)}<+\infty
$$

We clearly can choose a positive constant $\eta$ in Proposition 5.3 which suits to any data $\left(\tilde{b}_{0}, \tilde{u}_{0}, \tilde{f}\right)$ such that $\varepsilon\left\|\tilde{b}_{0}\right\|_{L^{\infty}} \leqslant 1 / 2,\left\|\tilde{b}_{0}\right\|_{B_{2}^{N / 2+\alpha}} \leqslant X_{M},\left\|\tilde{u}_{0}\right\|_{B_{2}^{N / 2-1+\alpha}} \leqslant X_{M}+\varepsilon_{0}^{\alpha_{N}} W_{M}$ and 
$\|\tilde{f}\|_{L_{T}^{1}\left(B_{2}^{N / 2-1+\alpha}\right)} \leqslant\|f\|_{L_{T}^{1}\left(B_{2}^{N / 2-1+\alpha}\right)}$. Let us remark now that

$$
\begin{gathered}
\tau=\min \left(\eta, \max \left\{t>0, \sum_{q \in \mathbb{Z}} 2^{q(N / 2-1+\alpha)}\left(\frac{1-\mathrm{e}^{-c t 2^{2 q}}}{c}\right)\right.\right. \\
\left.\left.\times\left(\left\|\Delta_{q} u\right\|_{L_{T_{\varepsilon}}^{\infty}\left(L^{2}\right)}+\left\|\Delta_{q} f\right\|_{L^{\infty}\left(L^{2}\right)}\right) \leqslant \eta\right\}\right)
\end{gathered}
$$

is positive according to (97).

Therefore, system (1) has a local solution $(\tilde{b}, \tilde{u}) \in E_{\varepsilon \nu, \tau}^{N / 2} \cap E_{\varepsilon \nu, \tau}^{N / 2+\alpha}$ with initial data $\left(b\left(T_{\varepsilon}-\tau / 2\right), u\left(T_{\varepsilon}-\tau / 2\right)\right)$ and external force $f\left(\cdot+T_{\varepsilon}-\tau / 2\right)$. Since uniqueness holds in the space $E_{\varepsilon \nu, T}^{N / 2} \cap E_{\varepsilon \nu, T}^{N / 2+\alpha}$, this means that $(b, u)$ may be continued beyond $T_{\varepsilon}$. In particular, $b$ is in $C\left(\left[0, T_{\varepsilon}+\tau / 2\left[\times \mathbb{R}^{N}\right)\right.\right.$. Since $\varepsilon|b(t, x)| \leqslant 1 / 2$ on $\left[0, T_{\varepsilon}[\right.$, we will still have $\varepsilon|b| \leqslant 3 / 4$ on a small time interval beyond $T_{\varepsilon}$. This stands in contradiction with the definition of $T_{\varepsilon}$. Therefore $T_{\varepsilon} \geqslant T_{0}$ for $\varepsilon \leqslant \varepsilon_{0}$.

\section{Some regularity results for incompressible viscous flows}

The first regularity result we need is well known in Sobolev spaces for example so that we shall only sketch the proof. The bound from below of the existence time is perhaps a bit less classical. A similar bound in the Sobolev spaces framework may be found in [4], Lemma 3.2.4. Let us state the result in question.

Proposition 6.1. - Let $N \geqslant 2, \mu>0$ and $\alpha \geqslant 0$. Let $v_{0} \in B_{2}^{N / 2-1} \cap B_{2}^{N / 2+\alpha-1}$ be a divergence free vector field and $f \in L^{1}\left(\mathbb{R}^{+} ; B_{2}^{N / 2-1} \cap B_{2}^{N / 2+\alpha-1}\right)$ be a divergence free external force. Then there exist two constants $c$ and $C$ depending only on $N$ and such that the incompressible Navier-Stokes equations

$$
\left\{\begin{array}{l}
\partial_{t} v+\mathcal{P}(v \cdot \nabla v)-\mu \Delta v=f \\
\left.v\right|_{t=0}=v_{0}
\end{array}\right.
$$

have a unique solution $v \in F_{T}^{N / 2} \cap F_{T}^{N / 2+\alpha}$ with a time $T$ bounded from below by

$$
\sup \left\{T^{\prime}>0, \sum_{q \in \mathbb{Z}} 2^{q(N / 2-1)}\left(1-\mathrm{e}^{-c \mu 2^{2 q} T^{\prime}}\right)^{1 / 2}\left(\left\|\Delta_{q} v_{0}\right\|_{L^{2}}+\left\|\Delta_{q} f\right\|_{L_{T^{\prime}}^{1}\left(L^{2}\right)}\right) \leqslant C \mu\right\} .
$$

In particular, $T$ is strictly positive and $T=+\infty$ if $\left\|v_{0}\right\|_{B_{2}^{N / 2-1}}+\|f\|_{L^{1}\left(B_{2}^{N / 2-1}\right)} \leqslant C \mu$.

Proof. - We shall follow the fixed point method used in [2]. Denote by $\mathrm{e}^{t \Delta}$ the semi-group of the heat equation. Let $v_{L} \in F^{N / 2} \cap F^{N / 2+\alpha}$ be the solution of

$$
\partial_{t} v_{L}-\mu \Delta v_{L}=f, \quad v_{L}(0)=v_{0}
$$

Assume that the time $T \in] 0,+\infty]$ has been chosen in such a way that

$$
\left\|v_{L}\right\|_{L_{T}^{2}\left(B_{2}^{N / 2}\right)} \leqslant \mu^{1 / 2} / 4 C
$$

for a constant $C$ to be defined below. 
Let $0<R<\mu /(4 C)$ and $R_{\alpha}=\mu^{1 / 2}\left\|v_{L}\right\|_{L_{T}^{2}\left(B_{2}^{N / 2+\alpha}\right)}$. Let $\mathcal{G}$ be the set of divergence free vector fields with coefficients in $F_{T}^{N / 2} \cap F_{T}^{N / 2+\alpha}$, and such that $\|v\|_{F_{T}^{N / 2}} \leqslant R$ and $\|v\|_{F_{T}^{N / 2+\alpha}} \leqslant R_{\alpha}$. Define the following function:

$$
\mathcal{F}(\bar{v}) \stackrel{\text { def }}{=}-\int_{0}^{t} \mathrm{e}^{(t-\tau) \mu \Delta} \mathcal{P} \operatorname{div}\left(\left(v_{L}+\bar{v}\right)(\tau) \otimes\left(v_{L}+\bar{v}\right)(\tau)\right) \mathrm{d} \tau .
$$

According to Propositions 7.3 and 1.4, $\mathcal{F}$ maps $F_{T}^{N / 2} \cap F_{T}^{N / 2+\alpha}$ into itself, and, for $\beta=0$ or $\alpha$, we have

$$
\|\mathcal{F}(\bar{v})\|_{F_{T}^{N / 2+\beta}} \leqslant C \mu^{-1}\left(\|\bar{v}\|_{F_{T}^{N / 2}}+\mu^{1 / 2}\left\|v_{L}\right\|_{L_{T}^{2}\left(B_{2}^{N / 2}\right)}\right)\left(\|\bar{v}\|_{F_{T}^{N / 2+\beta}}+\mu^{1 / 2}\left\|v_{L}\right\|_{L_{T}^{2}\left(B_{2}^{N / 2+\beta}\right)}\right)
$$

so that we easily gather that $\mathcal{F}$ maps $\mathcal{G}$ to $\mathcal{G}$. Similar computations show us that

$$
\begin{aligned}
& \|\mathcal{F}(\bar{v})-\mathcal{F}(\bar{w})\|_{F_{T}^{N / 2+\beta}} \\
& \quad \leqslant C \mu^{-1}\left(\left(\|\bar{v}\|_{F_{T}^{N / 2}}+\|\bar{w}\|_{F_{T}^{N / 2}}+2 \mu^{1 / 2}\left\|v_{L}\right\|_{L_{T}^{2}\left(B_{2}^{N / 2}\right)}\right)\|(\bar{v}-\bar{w})\|_{F_{T}^{N / 2+\beta}}\right. \\
& \left.\quad+\left(\|\bar{v}\|_{F_{T}^{N / 2+\beta}}+\|\bar{w}\|_{F_{T}^{N / 2+\beta}}+2 \mu^{1 / 2}\left\|v_{L}\right\|_{L_{T}^{2}\left(B_{2}^{N / 2+\beta}\right)}\right)\|(\bar{v}-\bar{w})\|_{F_{T}^{N / 2}}\right) .
\end{aligned}
$$

Denote $k=1 / 2+2 R C / \mu$ and $K=4 R_{\alpha} C / \mu$. According to the above inequality, we have for all $\eta>0$

$$
\begin{aligned}
& \|\mathcal{F}(\bar{v})-\mathcal{F}(\bar{w})\|_{F_{T}^{N / 2}}+\eta\|\mathcal{F}(\bar{v})-\mathcal{F}(\bar{w})\|_{F_{T}^{N / 2+\alpha}} \\
& \quad \leqslant(k+\eta K)\left(\|\bar{v}-\bar{w}\|_{F_{T}^{N / 2}}+\eta\|\bar{v}-\bar{w}\|_{F_{T}^{N / 2+\alpha}}\right) .
\end{aligned}
$$

Choosing $\eta$ so small that $k+\eta K<1$, we conclude that $\mathcal{F}$ is a contraction on $\mathcal{G}$ endowed with the norm $\|\cdot\|_{F_{T}^{0}}+\eta\|\cdot\|_{F_{T}^{N / 2+\alpha}}$. Denoting $v=v_{L}+\bar{v}$ where $\bar{v}$ is the unique fixed point of $\mathcal{F}$ in $\mathcal{G}$, we easily gather that $v$ solves (98).

Now, according to Proposition 2.3 in [7], we have, for two constants $c$ and $C_{1}$ depending only on $N$,

$$
\mu^{1 / 2}\left\|v_{L}\right\|_{L_{T}^{2}\left(B_{2}^{N / 2}\right)} \leqslant C_{1}\left(\sum_{q \in \mathbb{Z}} 2^{q(N / 2-1)}\left(1-\mathrm{e}^{-c \mu 2^{2 q} T}\right)^{1 / 2}\left(\left\|\Delta_{q} v_{0}\right\|_{L^{2}}+\left\|\Delta_{q} f\right\|_{L_{T}^{1}\left(L^{2}\right)}\right)\right) .
$$

According to Lebesgue theorem, the right-hand side tends to zero when $T$ tends to zero. Coming back to (99), this gives us a bound from below for the life span of $v$.

Uniqueness is classical and actually holds in a larger space: in $L_{\mathrm{loc}}^{\infty}\left(0, T ; \dot{H}^{N / 2-1}\right) \cap$ $L_{\text {loc }}^{2}\left(0, T ; \dot{H}^{N / 2}\right)$ for instance (see [3]).

It turns out that the quantity $\|v\|_{B_{\infty, \infty}^{0}}$ controls positive regularity for $v$. More precisely, we have

LEMma 6.2. - Let $s>0$ and $v \in F_{T}^{s}$ be a solution of (98). Then for all $0 \leqslant t<T$, the following estimate holds for a constant $C$ depending only on $s$ and a universal constant $\kappa$ :

$$
\|v(t)\|_{B_{2}^{s-1}}+\kappa \mu \int_{0}^{t}\|v(\tau)\|_{B_{2}^{s+1}} \mathrm{~d} \tau \leqslant \mathrm{e}^{C V(t)}\left(\left\|v_{0}\right\|_{B_{2}^{s-1}}+\int_{0}^{t} \mathrm{e}^{-C V(\tau)}\|f(\tau)\|_{B_{2}^{s-1}} \mathrm{~d} \tau\right),
$$

with $V(t) \stackrel{\text { def }}{=} \mu^{-1} \int_{0}^{t}\|v(\tau)\|_{B_{\infty, \infty}^{0}}^{2} \mathrm{~d} \tau$. 
Proof. - Using Bony decomposition, we have

$$
\partial_{t} v+\mathcal{P}\left(T_{v} \nabla v\right)-\mu \Delta v=f-\mathcal{P}\left(T_{\nabla v}^{\prime} v\right) .
$$

Apply Proposition 4.2 with $p=2$. Denoting $V(t) \stackrel{\text { def }}{=} \mu^{-1} \int_{0}^{t}\|v(\tau)\|_{B_{\infty}^{0}, \infty}^{2} \mathrm{~d} \tau$, we get for $0 \leqslant t<T$,

$$
\begin{aligned}
\|v(t)\|_{B_{2}^{s-1}}+\kappa \mu \int_{0}^{t}\|v(\tau)\|_{B_{2}^{s+1}} \mathrm{~d} \tau \leqslant & \mathrm{e}^{C V(t)}\left(\left\|v_{0}\right\|_{B_{2}^{s-1}}+\int_{0}^{t} \mathrm{e}^{-C V(\tau)}\|f(\tau)\|_{B_{2}^{s-1}} \mathrm{~d} \tau\right. \\
& \left.+\int_{0}^{t} \mathrm{e}^{-C V(\tau)}\left\|\mathcal{P}\left(T_{\nabla v}^{\prime} v\right)(\tau)\right\|_{B_{2}^{s-1}} \mathrm{~d} \tau\right) .
\end{aligned}
$$

Since $\left(T_{\nabla v}^{\prime} v\right)^{i}=T_{\partial_{j} v^{i}} v^{j}+\partial_{j} R\left(v^{i}, v^{j}\right)$, we get from Proposition 1.9 and interpolation,

$$
\begin{aligned}
& \mathrm{e}^{C(V(t)-V(\tau))}\left\|\mathcal{P}\left(T_{\nabla v}^{\prime} v\right)(\tau)\right\|_{B_{2}^{s-1}} \\
& \quad \leqslant C \mathrm{e}^{C(V(t)-V(\tau))}\|v(\tau)\|_{B_{\infty}^{0}, \infty}\|v(\tau)\|_{B_{2}^{s}}, \\
& \quad \leqslant C \mathrm{e}^{C(V(t)-V(\tau))}\|v(\tau)\|_{B_{\infty}^{0}, \infty}\|v(\tau)\|_{B_{2}^{s-1}}^{1 / 2}\|v(\tau)\|_{B_{2}^{s+1}}^{1 / 2}, \\
& \quad \leqslant \frac{C^{2} \mathrm{e}^{2 C(V(t)-V(\tau))}}{2 \kappa \mu}\|v(\tau)\|_{B_{\infty}^{0}, \infty}^{2}\|v(\tau)\|_{B_{2}^{s-1}}+\frac{\kappa \mu}{2}\|v(\tau)\|_{B_{2}^{s+1}}
\end{aligned}
$$

Plugging this latter inequality in (100), we conclude to the desired estimate.

We conclude this section with a global regularity result in the two-dimensional case.

THEOREM 6.3. - Suppose $N=2$ and $\alpha \geqslant 0$. Suppose that the initial velocity $v_{0}$ belongs to $B_{2}^{0} \cap B_{2}^{\alpha}$ and that $f \in L^{1}\left(\mathbb{R}^{+} ; B_{2}^{0} \cap B_{2}^{\alpha}\right)$. Then (98) has a unique global solution $v$ in $F^{1} \cap F^{1+\alpha}$ and satisfies moreover

$$
\|v\|_{F^{1} \cap F^{1+\alpha}} \leqslant \mathrm{e}^{C \mu^{-2}\left(\left\|v_{0}\right\|_{L^{2}}+\|f\|_{L^{1}\left(L^{2}\right)}\right)^{2}}\left(\left\|v_{0}\right\|_{B_{2}^{0} \cap B_{2}^{\alpha}}+\|f\|_{L^{1}\left(B_{2}^{0} \cap B_{2}^{\alpha}\right)}\right) .
$$

Proof. - Let us first notice that $B_{2}^{0} \hookrightarrow L^{2}$ so that actually $v_{0} \in L^{2}$ and $f \in L^{1}\left(\mathbb{R}^{+} ; L^{2}\right)$. In the case $f \in L^{2}\left(\mathbb{R}^{+} ; \dot{H}^{-1}\right)$, it is well known since a celebrated result of J. Leray (see [23]) that (98) has a unique global solution $v \in C_{b}\left(\mathbb{R}^{+} ; L^{2}\right) \cap L^{2}\left(\mathbb{R}^{+} ; \dot{H}^{1}\right)$ which satisfies the energy equality. Slight modifications of the proof show that a similar result holds if $f \in L^{1}\left(\mathbb{R}^{+} ; L^{2}\right)$ and that we have, for a universal constant $\kappa$,

$$
\|v(t)\|_{L^{2}}^{2}+\kappa \mu \int_{0}^{t}\|\nabla v(\tau)\|_{L^{2}}^{2} \mathrm{~d} \tau \leqslant\left(\left\|v_{0}\right\|_{L^{2}}+\int_{0}^{t}\|f(\tau)\|_{L^{2}} \mathrm{~d} \tau\right)^{2} .
$$

Now, according to Proposition 6.1, (98) has a local solution $v^{\prime}$ in $F_{T}^{1} \cap F_{T}^{1+\alpha}$ which, in view of uniqueness, must coincide with $v$ on $[0, T[$. Denote

$$
T^{\star}=\sup \left\{T>0, v \in F_{T}^{1} \cap F_{T}^{1+\alpha}\right\} .
$$

Since $\|v\|_{B_{\infty, \infty}^{0}} \leqslant C\|\nabla v\|_{L^{2}}$, Lemma 6.2 combined with (102) tells us that the norm of $v$ in $\bigcap_{T<T^{\star}} F_{T}^{1} \cap F_{T}^{1+\alpha}$ is bounded by the right-hand side of (101). 
Now, using Proposition 1.4, one easily gathers that $\partial_{t} v-\mu \Delta v \in L^{1}\left(0, T^{\star} ; B_{2}^{0}\right)$.

Since $v_{0} \in B_{2}^{0}$, Proposition 7.3 insures us that

$$
I^{\star} \stackrel{\text { def }}{=} \sum_{q \in \mathbb{Z}}\left\|\Delta_{q} v\right\|_{L_{T^{\star}}^{\infty}\left(L^{2}\right)}<+\infty .
$$

The end of the proof is standard. Suppose that $T^{\star}$ is finite. Then in view of Proposition 6.1, for any $t<T^{\star}$ one can define a solution $\tilde{v} \in F_{\tau}^{1} \cap F_{\tau}^{1+\alpha}$ to (98) with initial data $v(t)$. Moreover $\tau$ may be chosen depending only on $N, \mu, I^{\star}$ and $\|f\|_{L^{1}\left(L^{2}\right)}$, and not on $t$. By virtue of uniqueness, $\tilde{v}$ must coincide with $v$ on $\left[t, t+\tau\left[\right.\right.$. Hence, choosing $t=T^{\star}-\tau / 2$ contradicts the definition of $T^{\star}$.

\section{Appendix}

\subsection{A dispersive inequality}

For the convenience of the reader we here give a sketchy proof of Proposition 2.2. We actually give a more general and precise statement. We shall use the following notation for $s \in \mathbb{R}$, $1 \leqslant p, r \leqslant+\infty$ and $0<T \leqslant+\infty$ :

$$
\|z\|_{\widetilde{L}_{T}^{r}\left(B_{p}^{s}\right)} \stackrel{\text { def }}{=} \sum_{q \in \mathbb{Z}} 2^{q s}\left\|\Delta_{q} z\right\|_{L_{T}^{r}\left(L^{p}\right)} .
$$

Owing to Minkowski inequality, we have $\|z\|_{L_{T}^{r}\left(B_{p}^{s}\right)} \leqslant\|z\|_{\widetilde{L}_{T}^{r}\left(B_{p}^{s}\right)}$ and equality holds if $r=1$.

Proposition 7.1. - Let $(c, d)$ be a solution of $\left(W^{\varepsilon}\right)$. Then for any $s \in \mathbb{R}$ and positive $T$ (possibly infinite), the following estimate holds

$$
\begin{aligned}
& \|(c, d)\|_{\widetilde{L}_{T}^{r}\left(B_{p}^{s+N(1 / p-1 / 2)+1 / r}\right)} \\
& \quad \lesssim \varepsilon^{1 / r}\left\|\left(c_{0}, d_{0}\right)\right\|_{B_{2}^{s}}+\varepsilon^{1+1 / r-1 / \bar{r}^{\prime}}\|(F, G)\|_{\widetilde{L}_{T}^{\bar{r}^{\prime}}\left(B_{\bar{p}^{\prime}}^{s+N\left(1 / \bar{p}^{\prime}-1 / 2\right)+1 / \bar{r}^{\prime}}\right)}
\end{aligned}
$$

with

$$
\begin{array}{ll}
p \geqslant 2, & \frac{2}{r} \leqslant \min (1, \gamma(p)) \quad(r, p, N) \neq(2, \infty, 3), \\
\bar{p} \geqslant 2, \quad \frac{2}{\bar{r}} \leqslant \min (1, \gamma(\bar{p})) & (\bar{r}, \bar{p}, N) \neq(2, \infty, 3),
\end{array}
$$

where

$$
\gamma(q) \stackrel{\text { def }}{=}(N-1)\left(\frac{1}{2}-\frac{1}{q}\right), \quad \frac{1}{\bar{p}}+\frac{1}{\bar{p}^{\prime}}=1 \quad \text { and } \quad \frac{1}{\bar{r}}+\frac{1}{\bar{r}^{\prime}}=1 .
$$

Proof. - Denote $\Phi \stackrel{\text { def }}{=} t(c, d)$ and $H \stackrel{\text { def }}{=} t(F, G)$. Setting

$$
\Psi(t, x)=\Phi(\varepsilon t, x) \quad \text { and } \quad \mathcal{H}(t, x)=\varepsilon H(\varepsilon t, x)
$$

one easily checks that $\Psi$ solves $\left(W^{1}\right)$ with external force $\mathcal{H}$. Therefore, thanks to (5), (6) and (9), it suffices to treat the case $\varepsilon=1$. 
Denoting by $U(t)$ the group corresponding to the homogeneous equation $\left(W^{1}\right)$, we have in Fourier variables

$$
\mathcal{F}(U(t) \Phi)(\xi)=\left(\begin{array}{cc}
\cos (|\xi| t) & -\sin (|\xi| t) \\
\sin (|\xi| t) & \cos (|\xi| t)
\end{array}\right) \mathcal{F} \Phi_{0}(\xi)
$$

From it, we deduce exactly as for the wave equation (see for example [16]) that for any $g$ valued in $\mathbb{R}^{2}$ and such that $\mathcal{F} g$ is supported in the annulus $C(0,1 / 2,2)$, then

$$
\begin{aligned}
& \|U(t) g\|_{L^{2}} \leqslant\|g\|_{L^{2}}, \\
& \left\|U(s) U^{\star}(t) g\right\|_{L^{\infty}} \lesssim(1+|t-s|)^{-(N-1) / 2}\|g\|_{L^{1}} .
\end{aligned}
$$

According to [20], this yields Proposition 7.1 in the special case where the spectrum of the data is supported in the annulus $C(0,1 / 2,2)$.

In the general case, let us use Littlewood-Paley decomposition. Scaling arguments enable us to get the following estimate for each dyadic block

$$
2^{q(N(1 / p-1 / 2)+1 / r)}\left\|\Delta_{q} \Phi\right\|_{L_{T}^{r}\left(L^{p}\right)} \lesssim\left\|\Delta_{q} \Phi_{0}\right\|_{L^{2}}+2^{q\left(N\left(1 / \bar{p}^{\prime}-1 / 2\right)+1 / \bar{r}^{\prime}-1\right)}\left\|\Delta_{q} H\right\|_{L_{T}^{\bar{r}^{\prime}}\left(L^{\bar{p}^{\prime}}\right)} .
$$

We then conclude by multiplying both sides of the above inequality by $2^{q s}$ and by performing a summation on $\mathbb{Z}$.

Remark 7.2. - The fact that the summation on indices $q$ is done after the time integration for each dyadic block explains the reason why the norms in $\widetilde{L}_{T}^{r}\left(B_{p}^{s}\right)$ appear naturally. This is not specific to $\left(W^{\varepsilon}\right)$ and also applies to the heat equation (see Proposition 7.3 below).

\subsection{Some estimates for the heat equation}

In the proposition below, we state the smoothing properties we need in part 3 for the standard heat equation. The reader may refer to [5] for the proof of them.

Proposition 7.3. - Let $p \in[1,+\infty], 1 \leqslant s_{2} \leqslant s_{1} \leqslant+\infty$, and let $u$ solve

$$
\left\{\begin{array}{l}
\partial_{t} u-\mu \Delta u=f \\
u_{t=0}=u_{0}
\end{array}\right.
$$

Then there exists $C>0$ depending only on $N, \mu, s_{1}$ and $s_{2}$ such that

$$
\|u\|_{\widetilde{L}_{T}^{s_{1}}\left(B_{p}^{s+2 / s_{1}}\right)} \leqslant C\left\|u_{0}\right\|_{B_{p}^{s}}+C\|f\|_{\widetilde{L}_{T}^{s_{2}}\left(B_{p}^{s-2+2 / s_{2}}\right)} .
$$

Moreover u belongs to $C\left([0, T] ; B_{p}^{s}\right)$.

In Section 5, we need estimates for a heat equation with terms of order one.

Proposition 7.4. - Let $s \in] 1-N / 2,1+N / 2]$ and $z$ solving

$$
\left\{\begin{array}{l}
\partial_{t} z+\mathcal{P}(A \cdot \nabla z)+\mathcal{P}(z \cdot \nabla B)-\mu \Delta z=F \\
z_{t=0}=z_{0}
\end{array}\right.
$$


with $\operatorname{div} z_{0}=\operatorname{div} F=0$. There exist two constants $\kappa$ and $C=C(N, s)$ such that for all positive $T$ (possibly infinite), we have

$$
\|z\|_{L_{T}^{\infty}\left(B_{2}^{s-1}\right)}+\kappa \mu\|z\|_{L_{T}^{1}\left(B_{2}^{s+1}\right)} \leqslant \mathrm{e}^{C V(T)}\left(\left\|z_{0}\right\|_{B_{2}^{s-1}}+\int_{0}^{T} \mathrm{e}^{-C V(t)}\|F(\tau)\|_{B_{2}^{s-1}} \mathrm{~d} \tau\right)
$$

with $V(t) \stackrel{\text { def }}{=} \int_{0}^{t}\left(\|A(\tau)\|_{B_{2}^{N / 2+1}}+\|B(\tau)\|_{B_{2}^{N / 2+1}}\right) \mathrm{d} \tau$.

Proof. - Let us write the evolution equation for each dyadic block $\Delta_{q} z$ :

$$
\partial_{t} \Delta_{q} z+\Delta_{q} \mathcal{P}(A \cdot \nabla z)+\Delta_{q} \mathcal{P}(z \cdot \nabla B)-\mu \Delta_{q} z=\Delta_{q} F
$$

An energy method gives us

$$
\begin{aligned}
& \frac{1}{2} \frac{\mathrm{d}}{\mathrm{d} t}\left\|\Delta_{q} z\right\|_{L^{2}}^{2}+\mu\left\|\nabla \Delta_{q} z\right\|_{L^{2}}^{2} \\
& \quad=-\left(\Delta_{q} \mathcal{P}(A \cdot \nabla z) \mid \Delta_{q} z\right)-\left(\Delta_{q} \mathcal{P}(z \cdot \nabla B) \mid \Delta_{q} z\right)+\left(\Delta_{q} F \mid \Delta_{q} z\right) .
\end{aligned}
$$

Since Leray projector is symmetric and $\operatorname{div} z=0$, we have

$$
\left(\Delta_{q} \mathcal{P}(A \cdot \nabla z) \mid \Delta_{q} z\right)=\left(\Delta_{q}(A \cdot \nabla z) \mid \mathcal{P} \Delta_{q} z\right)=\left(\Delta_{q}(A \cdot \nabla z) \mid \Delta_{q} z\right) .
$$

Applying Lemma 5.1 in [6], we get for a positive sequence $\left(c_{q}\right)_{q \in \mathbb{Z}}$ such that $\sum_{q \in \mathbb{Z}} c_{q} \leqslant 1$,

$$
\left|\left(\Delta_{q} \mathcal{P}(A \cdot \nabla z) \mid \Delta_{q} z\right)\right| \lesssim c_{q} 2^{-q(s-1)}\|z\|_{B_{2}^{s-1}}\|A\|_{B_{2}^{N / 2+1}}\left\|\Delta_{q} z\right\|_{L^{2}} .
$$

Since $\operatorname{div} z=0$, Bony decomposition for the term $z \cdot \nabla B$ yields

$$
(z \cdot \nabla B)^{i}=T_{z^{j}} \partial_{j} B^{i}+T_{\partial_{j} B^{i}} z^{j}+\partial_{j} R\left(B^{i}, z^{j}\right) .
$$

Thanks to Proposition 1.9 and Lemma 1.2, we thus have

$$
\left\|\Delta_{q} \mathcal{P}(z \cdot \nabla B)\right\|_{L^{2}} \lesssim c_{q} 2^{-q(s-1)}\|z\|_{B_{2}^{s-1}}\|B\|_{B_{2}^{N / 2+1}}
$$

Plugging (104) and (105) in (103) and using Bernstein inequality, we gather

$$
\frac{1}{2} \frac{\mathrm{d}}{\mathrm{d} t}\left\|\Delta_{q} z\right\|_{L^{2}}^{2}+\kappa \mu 2^{2 q}\left\|\Delta_{q} z\right\|_{L^{2}}^{2} \leqslant\left\|\Delta_{q} z\right\|_{L^{2}}\left(\left\|\Delta_{q} F\right\|_{L^{2}}+C c_{q} 2^{-q(s-1)} V^{\prime}\|z\|_{B_{2}^{s-1}}\right) .
$$

Integrate in time and multiply both sides of the above inequality by $2^{q(s-1)}$. We get

$$
\begin{aligned}
& 2^{q(s-1)}\left\|\Delta_{q} z(t)\right\|_{L^{2}}+\kappa \mu \int_{0}^{t} 2^{q(s+1)}\left\|\Delta_{q} z(\tau)\right\|_{L^{2}} \mathrm{~d} \tau \\
& \quad \leqslant 2^{q(s-1)}\left\|\Delta_{q} z_{0}\right\|_{L^{2}}+\int_{0}^{t} 2^{q(s-1)}\left\|\Delta_{q} F(\tau)\right\|_{L^{2}} \mathrm{~d} \tau+C \int_{0}^{t} c_{q}(\tau) V^{\prime}(\tau)\|z(\tau)\|_{B_{2}^{s-1}} \mathrm{~d} \tau .
\end{aligned}
$$

Now, summing on $q$ and using Gronwall inequality enable us to conclude to the desired estimate. 


\subsection{Estimates for the convection term}

In this last section of the appendix, we state an estimate used in Section 4.

LEMma 7.5. - Let $A \in C^{\infty}\left(\mathbb{R}^{N} \backslash\{0\}\right)$ be a non-vanishing homogeneous function of degree $m \in \mathbb{R}$, and $s>0$. Then for any $z \in \mathcal{S}^{\prime}\left(\mathbb{R}^{N}\right)$ and $u \in B_{\infty, \infty}^{1-s}$, we have

$$
A(D) \Delta_{q}\left(T_{u^{j}} \partial_{j} z\right)=S_{q-1} u^{j} \partial_{j} A(D) \Delta_{q} z+R_{q}(A, z)
$$

with

$$
\left\|R_{q}(A, z)\right\|_{L^{2}} \lesssim 2^{q s}\|\nabla u\|_{B_{\infty}^{-s}, \infty} \sum_{\left|q^{\prime}-q\right| \leqslant 3}\left\|\Delta_{q^{\prime}} A(D) z\right\|_{L^{2}} .
$$

If $\nabla u \in L^{\infty}$, then we have

$$
\left\|R_{q}(A, z)\right\|_{L^{2}} \lesssim\|\nabla u\|_{L^{\infty}} \sum_{\left|q^{\prime}-q\right| \leqslant 3}\left\|\Delta_{q^{\prime}} A(D) z\right\|_{L^{2}}
$$

Proof. - The starting point is the following equality

$$
\begin{aligned}
A(D) \Delta_{q}\left(T_{u^{j}} \partial_{j} z\right)= & A(D) \Delta_{q} \sum_{\left|q^{\prime}-q\right| \leqslant 3} S_{q^{\prime}-1} u^{j} \Delta_{q^{\prime}} \partial_{j} z, \\
= & S_{q-1} u^{j} \partial_{j} A(D) \Delta_{q} z+A(D) \Delta_{q} \sum_{\left|q^{\prime}-q\right| \leqslant 3}\left(S_{q^{\prime}-1}-S_{q-1}\right) u^{j} \Delta_{q^{\prime}} \partial_{j} z \\
& +\sum_{\left|q^{\prime}-q\right| \leqslant 3}\left[A(D) \Delta_{q}, S_{q-1} u^{j}\right] \partial_{j} \Delta_{q^{\prime}} z .
\end{aligned}
$$

Note that, due to (4), the summations in the above equalities may indeed be restricted to the indices $q^{\prime}$ such that $\left|q^{\prime}-q\right| \leqslant 3$.

On the other hand, $A(D) \Delta_{q}=2^{q m} \theta\left(2^{-q} D\right)$ with $\theta \stackrel{\text { def }}{=} A \varphi$. Since $\theta$ does not vanish on a suitably thin annulus $C\left(0, R_{1}, R_{2}\right)$, Bernstein inequality yields

$$
\left\|A(D) \Delta_{q} z\right\|_{L^{2}} \approx 2^{q m}\left\|\Delta_{q} z\right\|_{L^{2}} .
$$

This enables us to write the following calculations for $s \geqslant 0$ :

$$
\begin{aligned}
& \left\|A(D) \Delta_{q} \sum_{\left|q^{\prime}-q\right| \leqslant 3}\left(S_{q^{\prime}-1}-S_{q-1}\right) u^{j} \Delta_{q^{\prime}} \partial_{j} z\right\|_{L^{2}} \\
& \lesssim 2^{q m} \sum_{\left|q^{\prime}-q\right| \leqslant 3} 2^{q^{\prime}}\left\|\left(S_{q^{\prime}-1}-S_{q-1}\right) u\right\|_{L^{\infty}}\left\|\Delta_{q^{\prime}} z\right\|_{L^{2}}, \\
& \lesssim 2^{q s}\|u\|_{B_{\infty, \infty}^{1-s}} \sum_{\left|q^{\prime}-q\right| \leqslant 3} 2^{q^{\prime} m}\left\|\Delta_{q^{\prime}} z\right\|_{L^{2}}, \\
& \lesssim 2^{q s}\|\nabla u\|_{B_{\infty}^{-s}, \infty} \sum_{\left|q^{\prime}-q\right| \leqslant 3}\left\|\Delta_{q^{\prime}} A(D) z\right\|_{L^{2}} .
\end{aligned}
$$

Let $h \stackrel{\text { def }}{=} \mathcal{F}^{-1} \theta$. The last term in the right-hand side of (106) may be bounded thanks to the first order Taylor's formula: 


$$
\begin{aligned}
& {\left[A(D) \Delta_{q}, S_{q-1} u^{j}\right] \partial_{j} \Delta_{q^{\prime}} z(x)} \\
& \quad=2^{q(m-1)} \int_{\mathbb{R}^{N}} \int_{0}^{1} h(y) y^{k} S_{q-1} \partial_{k} u^{j}\left(x-2^{-q} \tau y\right) \Delta_{q^{\prime}} \partial_{j} z\left(x-2^{-q} y\right) \mathrm{d} \tau \mathrm{d} y .
\end{aligned}
$$

Making use of convolution inequalities and (107), we eventually get

$$
\begin{aligned}
\left\|\left[A(D) \Delta_{q}, S_{q-1} u^{j}\right] \partial_{j} \Delta_{q^{\prime}} z\right\|_{L^{2}} & \lesssim 2^{q(m-1)}\left\|\Delta_{q^{\prime}} \nabla z\right\|_{L^{2}}\left\|\nabla S_{q-1} u\right\|_{L^{\infty}}, \\
& \lesssim\left\|\Delta_{q^{\prime}} A(D) z\right\|_{L^{2}}\left\|\nabla S_{q-1} u\right\|_{L^{\infty}} .
\end{aligned}
$$

We conclude by using that

$$
\left\|\nabla S_{q-1} u\right\|_{L^{\infty}} \lesssim 2^{q s}\|\nabla u\|_{B_{\infty}^{-s}, \infty} \quad \text { if } s>0 \quad \text { and } \quad\left\|\nabla S_{q-1} u\right\|_{L^{\infty}} \lesssim\|\nabla u\|_{L^{\infty}}
$$

\section{Acknowledgements}

This work was performed while visiting the Institute for Advanced Study, and was partly supported by the NSF grant DMS 97-29992 and the NEC Research Institute, Inc.

\section{REFERENCES}

[1] BONY J.-M., Calcul symbolique et propagation des singularités pour les équations aux dérivées partielles non linéaires, Ann. Sci. École Norm. Sup. 14 (1981) 209-246.

[2] Cannone M., Ondelettes, paraproduits et Navier-Stokes, Nouveaux essais, Diderot éditeurs, 1995.

[3] Chemin J.-Y., Remarques sur l'existence globale pour le système de Navier-Stokes incompressible, SIAM J. Math. Anal. 23 (1992) 20-28.

[4] Chemin J.-Y., About Navier-Stokes system, Prépublication du Laboratoire d'analyse numérique de Paris 6, R96023, 1996.

[5] Chemin J.-Y., Théorèmes d'unicité pour le système de Navier-Stokes tridimensionnel, J. Anal. Math. 77 (1999) 27-50.

[6] DANCHIN R., Global existence in critical spaces for compressible Navier-Stokes equations, Inventiones Math. 141 (2000) 579-614.

[7] DANChin R., Local theory in critical spaces for compressible viscous and heat-conductive gases, Comm. Partial Differential Equations 26 (2001) 1183-1233.

[8] DANCHIN R., Global existence in critical spaces for compressible viscous and heat-conductive gases, Arch. Rational Mech. Anal. 160 (2001) 1-39.

[9] DANCHIN R., Zero Mach number limit for compressible flows with periodic boundary conditions, submitted.

[10] DANChin R., On the uniquiness in critical spaces for compressible Navier-Stokes equations, Nonlinear Differential Equations Appl., to appear.

[11] Desjardins B., Grenier E., Low Mach number limit of viscous compressible flows in the whole space, Roy. Soc. London Proc. Series A 455 (1986) (1999) 2271-2279.

[12] Desjardins B., Grenier E., Lions P.-L., Masmoudi N., Incompressible limit for solutions of the isentropic Navier-Stokes equations with Dirichlet boundary conditions, J. Math. Pures Appl. 78 (1999) 461-471.

[13] Fabrie P., Galusinski C., The slightly compressible Navier-Stokes equations revisited, Preprint, Mathématiques Appliquées de Bordeaux, France, 1998.

[14] Fujita H., Kato T., On the Navier-Stokes initial value problem I, Arch. Rational Mech. Anal. 16 (1964) 269-315.

[15] Gallagher I., A remark on smooth solutions of the weakly compressible periodic Navier-Stokes equations, Prépublication Université Paris-Sud, Mathématiques, 1999. 
[16] Ginibre J., Velo G., Generalized Strichartz inequalities for the wave equation, J. Funct. Anal. 133 (1995) 50-68.

[17] Hagstrom T., Lorenz J., All-time existence of classical solutions for slightly compressible flows, SIAM J. Math. Anal. 29 (1998) 652-672.

[18] Hoff D., The zero-Mach limit of compressible flows, Comm. Math. Phys. 192 (1998) 543-554.

[19] Hoff D., Zumbrun K., Multi-dimensional diffusion waves for the Navier-Stokes equations of compressible flow, Indiana Univ. Math. J. 44 (1995) 603-676.

[20] KeEl M., Tao T., Endpoint Strichartz estimates, Amer. J. Math. 120 (1998) 955-980.

[21] Klainerman S., Majda A., Compressible and incompressible fluids, Comm. Pure Appl. Math. 35 (1982) 629-651.

[22] Kreiss H.-O., Lorenz J., NAughton M., Convergence of the solutions of the compressible to the solutions of the incompressible Navier-Stokes equations, Adv. Pure Appl. Math. 12 (1991) 187-214.

[23] Leray J., Sur le mouvement d'un liquide visqueux remplissant l'espace, Acta Math. 63 (1934) 193248.

[24] LiN C., On the incompressible limit of the compressible Navier-Stokes equations, Comm. Partial Differential Equations 20 (1995) 677-707.

[25] Lions P.-L., Mathematical Topics in Fluid Dynamics, Vol. 1. Incompressible Models, Oxford University Press, 1996.

[26] Lions P.-L., Mathematical Topics in Fluid Dynamics, Vol. 2. Compressible Models, Oxford University Press, 1998.

[27] Lions P.-L., MAsmoudi N., Incompressible limit for a viscous compressible fluid, J. Math. Pures Appl. 77 (1998) 585-627.

[28] Lions P.-L., Masmoudi N., Une approche locale de la limite incompressible, C. R. Acad. Sci. Paris, Série I 329 (1999) 387-392.

[29] Peetre J., New Thoughts on Besov Spaces, Duke University Mathematical Series, Vol. 1, Durham N.C., 1976.

[30] Runst T., Sickel W., Sobolev Spaces of Fractional Order, Nemytskij Operators, and Nonlinear Partial Differential Equations, de Gruyter Series in Nonlinear Analysis and Applications, Vol. 3, Walter de Gruyter, Berlin, 1996.

[31] STRICHARTZ R., Restriction of Fourier transform to quadratic surfaces and decay of solutions of wave equations, Duke Math. J. 44 (1977) 705-774.

[32] UKAI S., The incompressible limit and the initial layer of the compressible Euler equation, J. Math. Kyoto Univ. 26 (1986) 323-331.

\footnotetext{
Raphaël DANCHIN

Laboratoire d'Analyse Numérique,

Université Paris 6,

175 rue du Chevaleret,

75013 Paris, France

E-mail: danchin@ann.jussieu.fr
} 